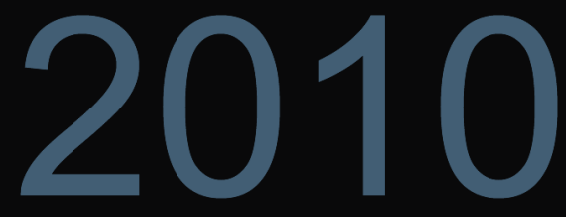

\title{
Kansas City Plant Annual IIIness and Injury Surveillance Report
}

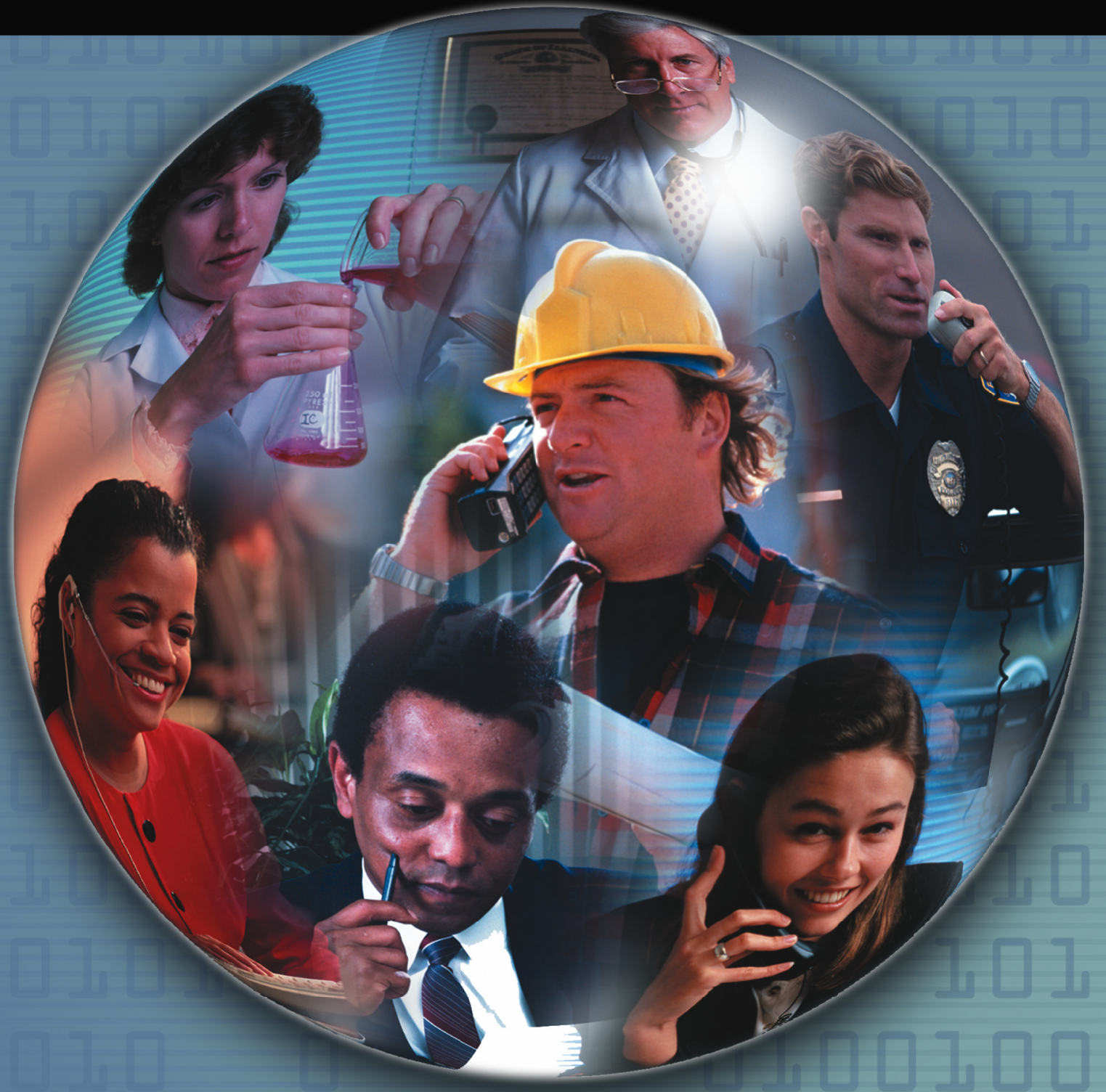




\section{Kansas City Plant 2010 Illness and Injury Surveillance Report}

Questions or comments about this report or the Illness and Injury Surveillance Program (IISP) may be directed to:

E-mail:

Dr. Cliff Strader at cliff.strader@hq.doe.gov or Dr. Bonnie Richter at bonnie.richter@hq.doe.gov

or direct letters to:

Mail Stop HS-13 / GTN Building

U.S. Department of Energy

1000 Independence Avenue, S.W.

Washington, DC 20585-0270

Additional information about the Department of Energy's Office of Illness and Injury Prevention Programs, the IISP, and annual reports for DOE sites participating in this program can be found at:

http://www.hss.energy.gov/healthsafety/WSHP/epi/surv/

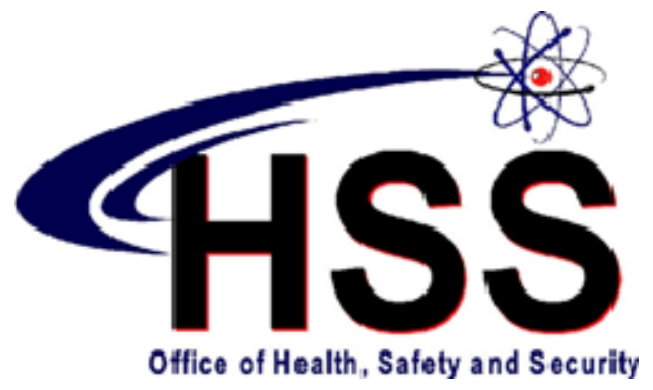

ACKNOWLEDGEMENT

LifeART images copyright 2000 Lippincott Williams \& Wilkins. All rights reserved.

This document was produced under contract number DE-AC05-06OR23100 between the

U.S. Department of Energy and Oak Ridge Associated Universities. 


\section{Kansas City Plant \\ 2010 Illness and Injury Surveillance Report}

At A Glance

NOTE: In previous years, the number of days absent or lost and the age of the work force were reported as averages. Beginning in 2010, they are being reported as medians. A median is the value in a population that half the values exceed and half fall short of it. The median is less affected than the average when the distribution contains a few very large or very small values.

A total of 2,501 Kansas City Plant employees, 612 (24 percent) women and 1,889 (76 percent) men, were included in illness and injury surveillance in 2010, a 2 percent decrease from 2009. The ratio of women to men has been constant since 2006 and is similar to the ratio seen among all DOE IISP sites from 1999 to 2008.

Crafts workers had the highest absence rate among men, and Security and Fire workers had the highest absence rate among women in 2010; male Security and Fire workers and female Administrative Support workers had the lowest rates. This is the first year since 2006 that male Crafts workers have had the highest absence rate; Service workers had the highest absence rates among men from 2006 to 2009. Women in the Security and Fire occupational group have had the highest absence rate for 4 of the 5 years since 2006 .

Women lost 1,365 calendar days due to illness and injury, about a 37 percent decrease over the calendar days lost in 2009. The median length of absence in 2010 was 16 days for women, the same as in 2009. Musculoskeletal conditions (24 percent), digestive conditions (13 percent), and injuries (10 percent) accounted for 47 percent of all reported diagnoses among women.

Men lost 4,470 calendar days due to illness and injury, 13 percent more calendar days lost than in 2009. In 2010, the median length of absence for men was 27 days, 10 days longer than in 2009. Fifty-five percent of all reported diagnoses among men were due to musculoskeletal conditions (25 percent), injuries (18 percent), and digestive conditions (12 percent). 
In 2010, the rate of all diagnoses combined decreased 30 percent for women and 40 percent for men compared with 2009 rates. The decreases appear to be due to fewer diagnoses for cancer, the respiratory system, and unspecified symptoms among women and for musculoskeletal and respiratory conditions, injuries, and unspecified symptoms among men.

Among men, Crafts workers had the highest rate for all diagnoses combined, which was twice the rate in 2009 , but the increase was not due to any particular illness or injury category. Technical Support workers had the highest rate for all illnesses and injuries combined for women. Among both men and women, the rate among Administrative Support workers was the lowest among all occupational groups in 2010.

Five sentinel occupational health event diagnoses were reported. Carpal tunnel syndrome accounted for 3 of these diagnoses, reported by 2 women and 1 man in the Service and Security and Fire occupational groups; all were aged 50 or older.

There were 5 OSHA-recordable events among men and 4 OSHA-recordable events among women at the Kansas City Plant in 2010. All 9 events were reported in workers aged 40 or older. Service workers had the highest rate of OSHA events among both men and women (almost 2 events per 100 workers and nearly 3 events per 100 workers, respectively).

Injuries (43 percent) and musculoskeletal conditions (33 percent) accounted for 76 percent of the OSHA diagnoses. Falls were the most frequent type of accident among women, while other accidents, mainly being struck by an object, were most common among men.

OsHA events were responsible for 85 days of restricted duty in 2010 , representing an 81 percent decrease from restricted days in 2009; there were no days lost due to an OSHA event in 2010. One worker each from the Crafts, Line Operators, and Service occupational groups were responsible for all 85 restricted days; all workers were aged 50 or older. 
The Kansas City Plant

Work Force - 2010

The Work Force by Gender and Age ............ 1

The Work Force by Gender and Job

Category .. 1

\section{Number and Length of Absences}

Absence Rate by Gender and Age 2

Number of Days Absent by

Gender and Age .. 2

Absence Rate by Job Category

and Gender .. 3

Median Duration of Absence by

Job Category and Gender. .. 3

\section{Diagnostic Categories}

Number of Diagnoses and Lost Calendar Days by Diagnostic Category

(Categorized by ICD-9-CM) and Gender . .4

Common Diagnoses Among Female

Workers in 2010 . . .5

Common Diagnoses Among Male

Workers in 2010 . 6

Number of Most Frequently Reported Diagnoses by Job Category and Gender 7

\section{Rates of Disease Occurrence}

Rates for All Illnesses and Injuries Combined by Job Category, Gender, and Age

Rates for Selected Diagnostic Categories by Job Category, Gender, and Age 8

\section{Time Trends}

Age-Adjusted Rates for All Diagnoses Combined Among Women and Men from 2006 to 2010
Age-Adjusted Rates for Selected Diagnostic Categories Among Women and Men from 2006 to 2010

Age-Adjusted Rates for All Diagnoses

Combined Among Women and Men by Job

Category from 2006 to $2010 \ldots \ldots \ldots \ldots \ldots \ldots \ldots \ldots . \ldots 12$

\section{Sentinel Health Events for Occupations (SHEOs)}

Characteristics of SHEOs by Gender 13

SHEO Diagnoses by Gender 13

\section{Occupational Safety and Health Administration (OSHA)-Recordable Events}

OSHA-Recordable Events by Gender and Age. 14

OSHA-Recordable Events by Job

Category and Gender 14

\section{Diagnostic and Accident Categories for OSHA-Recordable Events}

OSHA-Recordable Diagnoses by

Diagnostic Category and Gender

OSHA-Recordable Accidents by Type

and Gender

\section{Rates of OSHA-Recordable Events}

OSHA-Recordable Rates by Age and Job

Categories Among Women, All Diagnoses

Combined

OSHA-Recordable Rates by Age and Job

Categories Among Men, All Diagnoses

Combined

Time Trends for OSHA-Recordable Events

Age-Adjusted Rates for All OSHA-Recordable Diagnoses Combined Among Women and Men by Job Category from 2006 to 2010 .

\section{Appendices}

Appendices A-W 19 
The Kansas City Plant Work Force - 2010

Figure 1. The Work Force by Gender and Age

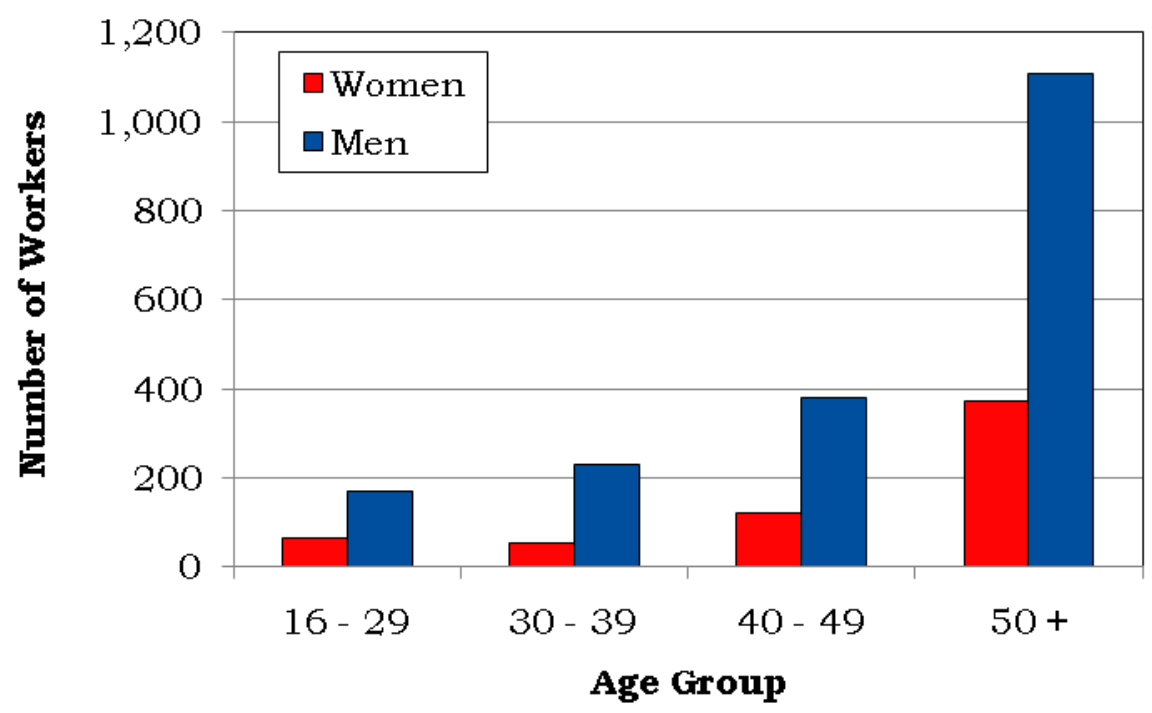

Figure 2. The Work Force by Gender and Job Category

\begin{tabular}{|l|c|c|}
\hline \multicolumn{1}{|c|}{ Job Category } & Women & M en \\
\hline Profe ssional & 155 & $\mathbf{8 3 9}$ \\
& $25 \%$ & $44 \%$ \\
\hline \multirow{2}{*}{ Administrative Support } & 245 & 237 \\
& $40 \%$ & $13 \%$ \\
\hline \multirow{2}{*}{ Technical Support } & 62 & 253 \\
& $10 \%$ & $13 \%$ \\
\hline \multirow{2}{*}{ Service } & 36 & 58 \\
& $6 \%$ & $3 \%$ \\
\hline \multirow{2}{*}{ Security and Fire } & 6 & 59 \\
& $1 \%$ & $3 \%$ \\
\hline \multirow{2}{*}{ Crafts } & 18 & 294 \\
& $3 \%$ & $16 \%$ \\
\hline \multirow{2}{*}{ Line Operators } & 90 & 149 \\
& $15 \%$ & $\mathbf{8} \%$ \\
\hline \multirow{2}{*}{ Total } & 612 & $\mathbf{1 , 8 8 9}$ \\
& $100 \%$ & $100 \%$ \\
\hline
\end{tabular}




\section{Number and Length of Absences}

Figure 3. Absence Rate by Gender and Age

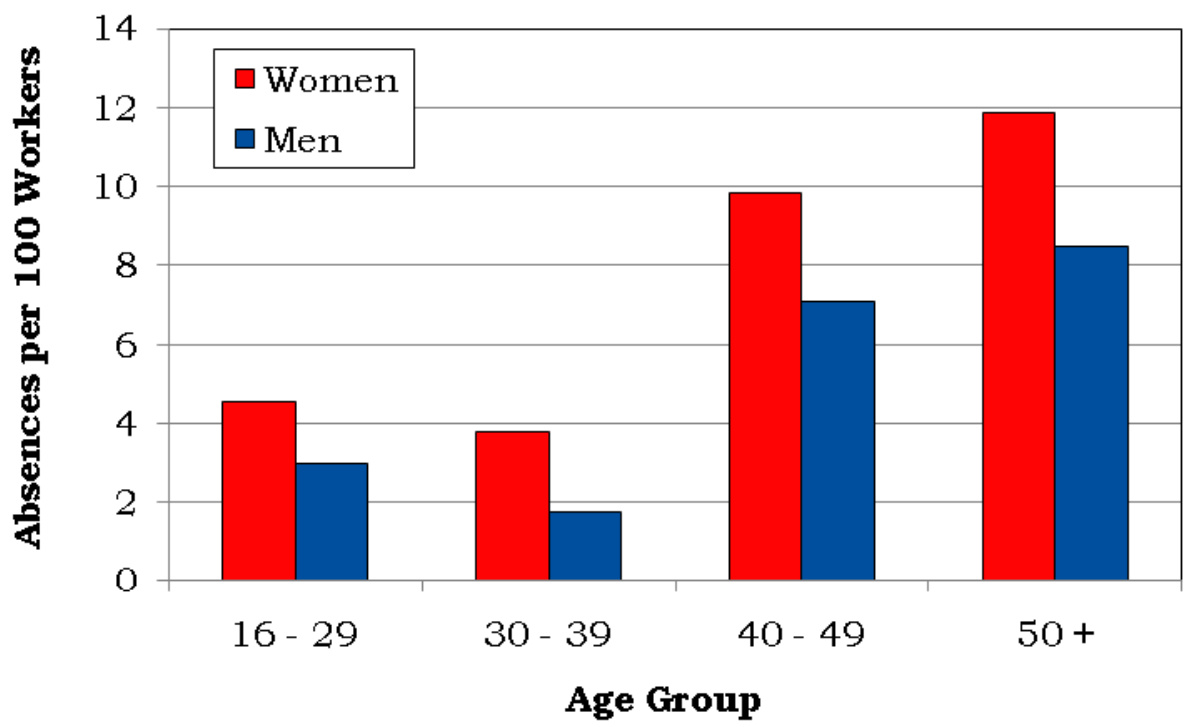

Figure 4. Number of Days Absent by Gender and Age

\begin{tabular}{|c|c|c|c|c|}
\hline \multirow{2}{*}{ Gender } & \multirow{2}{*}{ Age } & \multirow{2}{*}{$\begin{array}{c}\text { Number of } \\
\text { Absences }\end{array}$} & \multicolumn{2}{|c|}{ Number of Days Absent } \\
\hline & & & Total & Median \\
\hline \multirow{5}{*}{ Women* } & $16-29$ & 3 & 67 & 13 \\
\hline & $30-39$ & 2 & 30 & 15 \\
\hline & $40-49$ & 12 & 264 & 11 \\
\hline & $50+$ & 44 & 1,004 & 17 \\
\hline & Total & 61 & 1,365 & 16 \\
\hline \multirow{5}{*}{ Men } & $16-29$ & 5 & 158 & 24 \\
\hline & $30-39$ & 4 & 87 & 22 \\
\hline & $40-49$ & 27 & 718 & 13 \\
\hline & $50+$ & 94 & 3,507 & 29 \\
\hline & Total & 130 & 4,470 & 27 \\
\hline
\end{tabular}

*Normal pregnancies were excluded from absences for women. 
Figure 5. Absence Rate by Job Category and Gender

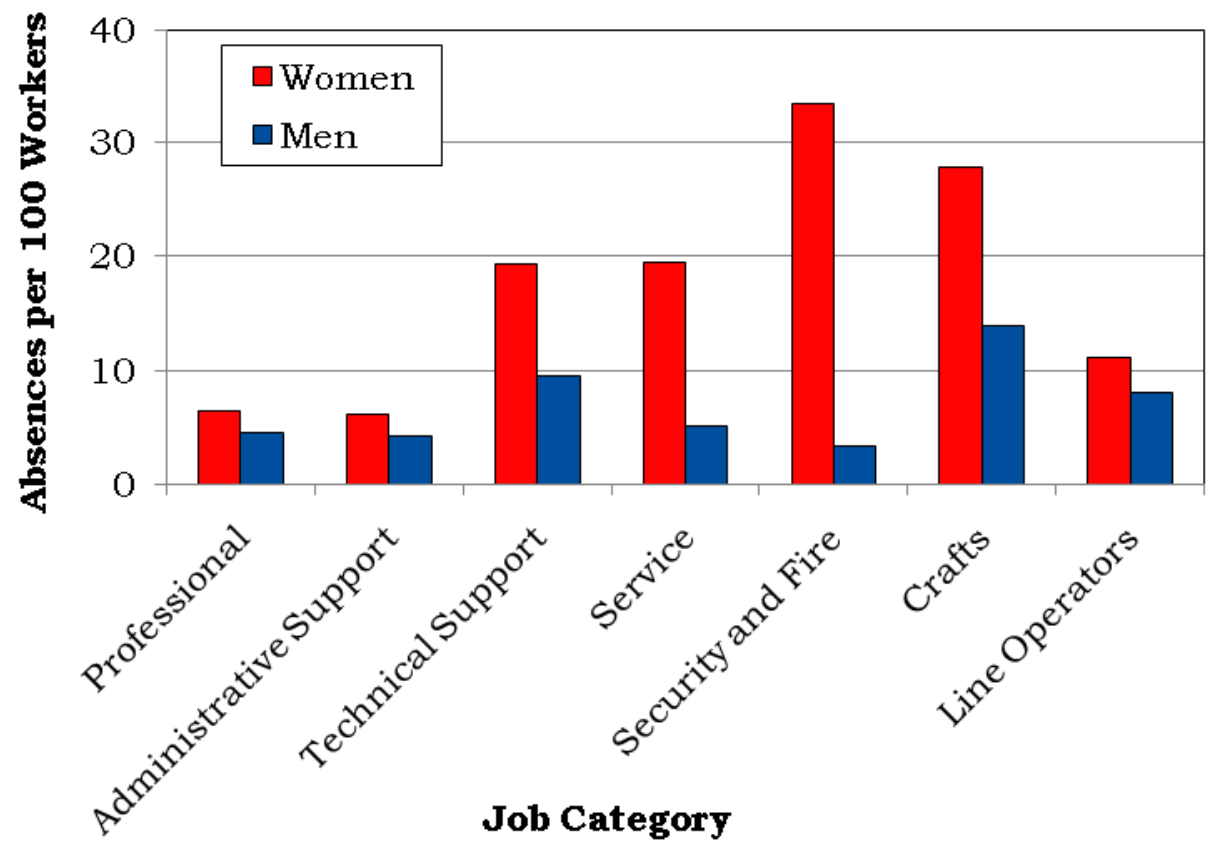

Figure 6. Median Duration of Absence by Job Category and Gender

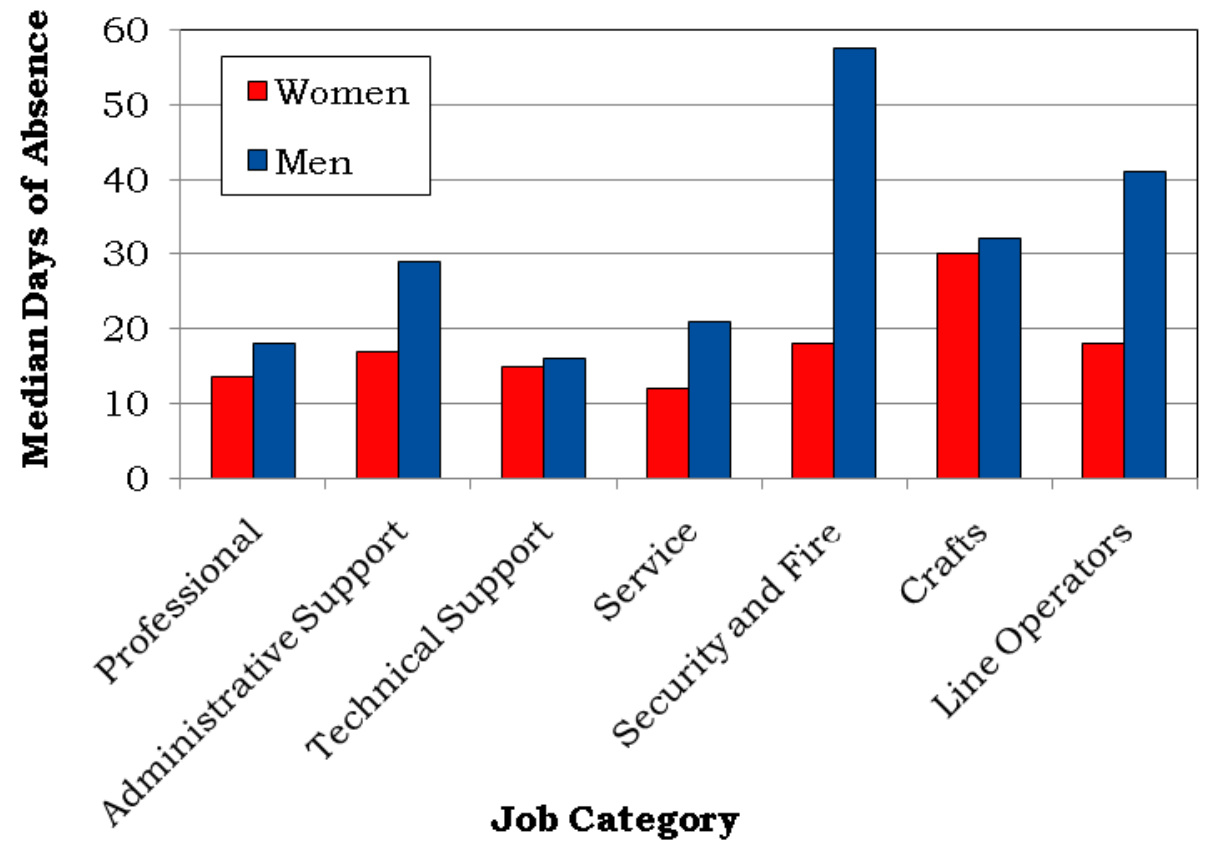




\section{Diagnostic Categories}

\section{Figure 7. Number of Diagnoses and Lost Calendar Days by Diagnostic Category (Categorized by ICD-9-CM) and Gender}

\begin{tabular}{|c|c|c|c|c|}
\hline \multirow[b]{2}{*}{ Diagnostic Category } & \multicolumn{2}{|c|}{ Women } & \multicolumn{2}{|c|}{ Men } \\
\hline & $\begin{array}{l}\text { Number of } \\
\text { Diagnoses }\end{array}$ & $\begin{array}{l}\text { Number } \\
\text { of Lost } \\
\text { Calendar } \\
\text { Days }\end{array}$ & $\begin{array}{l}\text { Number of } \\
\text { Diagnoses }\end{array}$ & $\begin{array}{c}\text { Number } \\
\text { of Lost } \\
\text { Calendar } \\
\text { Days }\end{array}$ \\
\hline Benign Growths & 2 & 23 & 0 & 0 \\
\hline Blood & 1 & 26 & 0 & 0 \\
\hline Cancer & 1 & 3 & 12 & 661 \\
\hline Digestive & 11 & 196 & 18 & 383 \\
\hline Endocrine / Me tabolic & 4 & 37 & 1 & 10 \\
\hline Existing Birth Condition & 0 & 0 & 0 & 0 \\
\hline Genitourinary & 5 & 137 & 5 & 271 \\
\hline Heart/Circulatory & 4 & 110 & 17 & 629 \\
\hline Infections/Parasites & 1 & 19 & 1 & 14 \\
\hline Injury & 9 & 246 & 28 & 812 \\
\hline Miscarriage & 0 & 0 & NA & NA \\
\hline Musculoskeletal & 21 & 539 & 38 & 1,351 \\
\hline Nervous System & 6 & 135 & 9 & 408 \\
\hline Psychological & 6 & 30 & 7 & 142 \\
\hline Respiratory & 5 & 64 & 7 & 84 \\
\hline Skin & 2 & 67 & 2 & 35 \\
\hline Unspecified Symptoms & 8 & 68 & 9 & 124 \\
\hline
\end{tabular}

Note: Lost calendar days for each absence are counted more than once when multiple diagnoses occur in different diagnostic categories for the same absence. 
Figure 8. Common Diagnoses Among Female Workers in 2010

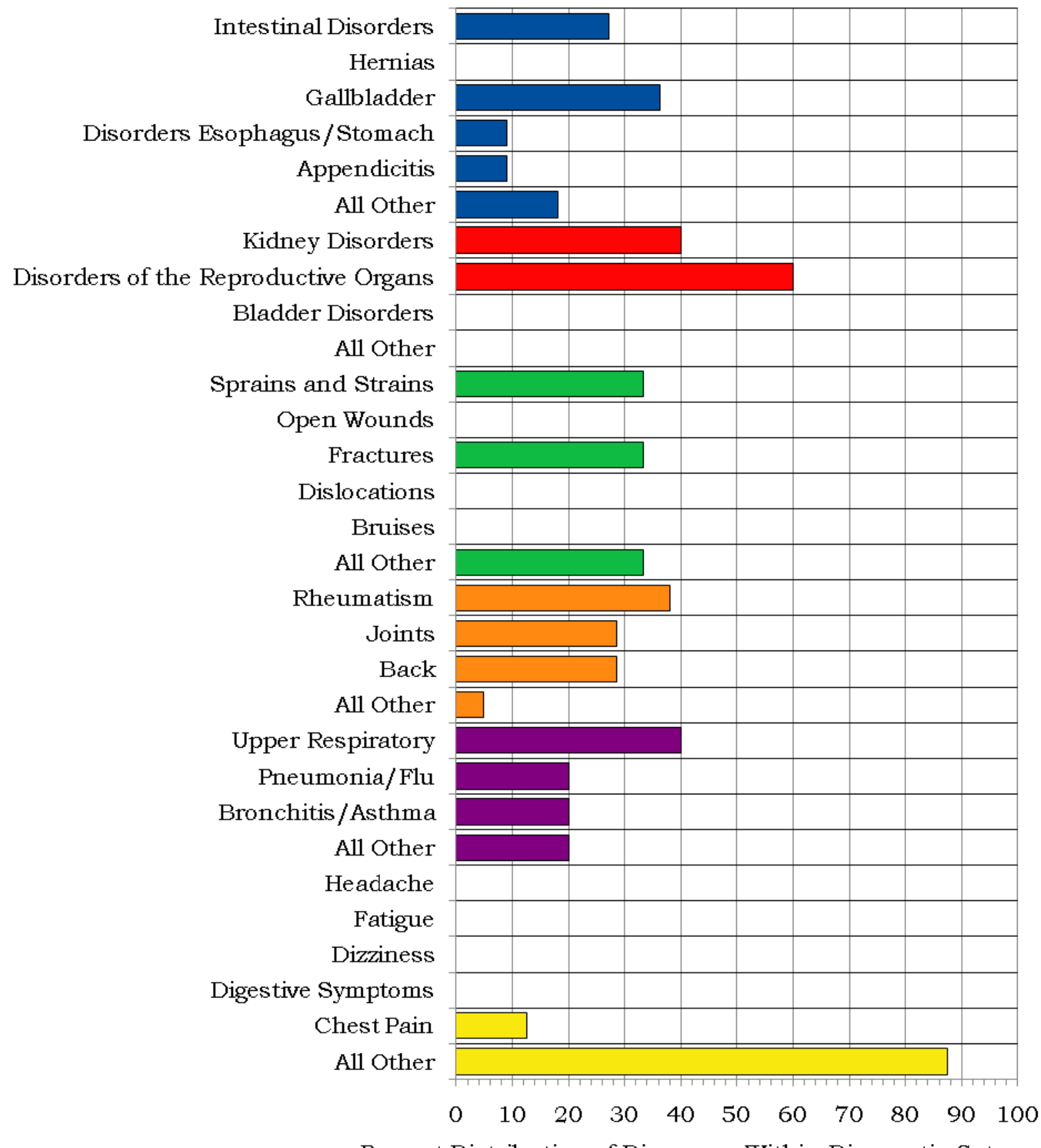

Percent Distribution of Diagnoses Within Diagnostic Category

Digestive, 11 Diagnoses

Genitourinary, 5 Diagnoses

Injury, 9 Diagnoses
Musculoskeletal, 21 Diagnoses

Respiratory, 5 Diagnoses

Unspecified Symptoms, 8 Diagnoses 


\section{Figure 9. Common Diagnoses Among Male Workers in 2010}

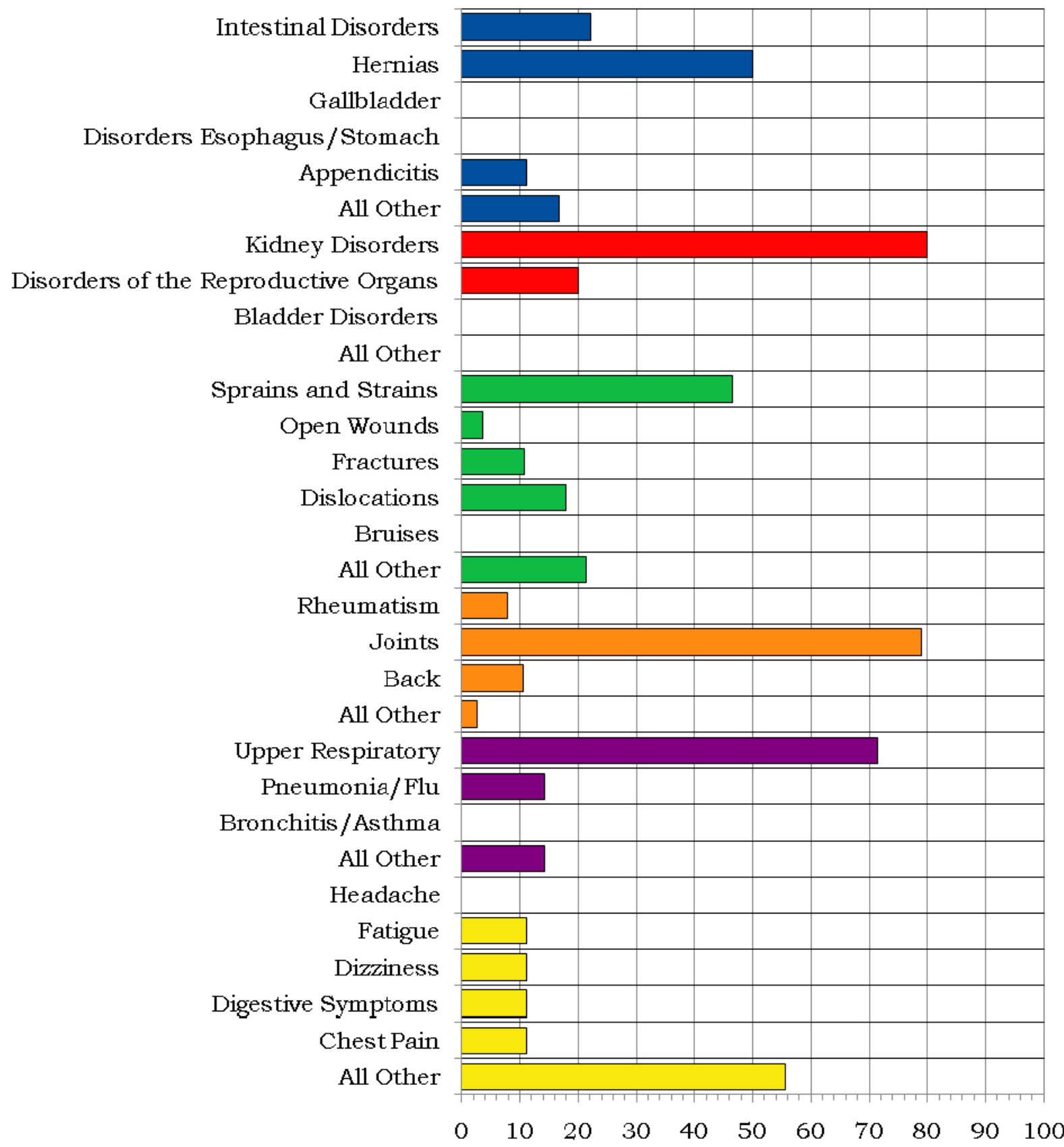

Percent Distribution of Diagnoses Within Diagnostic Category

Digestive, 18 Diagnoses

Genitourinary, 5 Diagnoses

Injury, 28 Diagnoses
Musculoskeletal, 38 Diagnoses

Respiratory, 7 Diagnoses

Unspecified Symptoms, 9 Diagnoses 


\section{Figure 10. Number of Most Frequently Reported Diagnoses by Job Category and Gender}

\begin{tabular}{|c|c|c|c|c|}
\hline Job Category & Men & & Women & \\
\hline \multirow{4}{*}{ Professional } & M usculoskeletal & 13 & Genitourinary & 3 \\
\hline & Injury & 11 & Nervous System & 2 \\
\hline & Digestive & 6 & Unspecified Symptoms & 2 \\
\hline & & & Heart/Circulatory & 2 \\
\hline \multirow{3}{*}{ Administrative Support } & M usculoskeletal & 3 & M usculoskeletal & 6 \\
\hline & Digestive & 3 & Injury & 5 \\
\hline & Cancer & 3 & Digestive & 4 \\
\hline \multirow{5}{*}{ Technical Support } & Heart/Circulatory & 4 & Unspecified Symptoms & 5 \\
\hline & Cancer & 4 & Psychological & 4 \\
\hline & M usculoskeletal & 3 & Endocrine/M etabolic & 3 \\
\hline & Digestive & 3 & & \\
\hline & Nervous System & 3 & & \\
\hline \multirow{5}{*}{ Service } & M usculoskeletal & 1 & M usculoskeletal & 3 \\
\hline & Injury & 1 & Digestive & 2 \\
\hline & Digestive & 1 & Nervous System & 1 \\
\hline & Nervous System & 1 & Genitourinary & 1 \\
\hline & skin & 1 & Respiratory & 1 \\
\hline \multirow{2}{*}{ Security and Fire } & Injury & 1 & Injury & 1 \\
\hline & Heart/Circulatory & 1 & Nervous System & 1 \\
\hline \multirow{3}{*}{ Crafts } & Musculoskeletal & 15 & M usculoskeletal & 3 \\
\hline & Injury & 8 & Respiratory & 2 \\
\hline & Heart/Circulatory & 7 & Digestive & 1 \\
\hline \multirow{3}{*}{ Line Operators } & Injury & 5 & M usculoskeletal & 6 \\
\hline & M usculoskeletal & 3 & Digestive & 3 \\
\hline & Cancer & 2 & Psychological & 2 \\
\hline
\end{tabular}




\section{Rates of Disease Occurrence}

Figure 11. Rates for All Illnesses and Injuries Combined by Job Category, Gender, and Age

\begin{tabular}{|c|c|c|c|c|}
\hline \multirow{2}{*}{$\begin{array}{c}\text { All Illnesses \& } \\
\text { Injuries Combined }\end{array}$} & \multicolumn{4}{|c|}{ Rate per 1,000} \\
\hline & Job Category & Age & Men & Women \\
\hline & \multirow{2}{*}{ Professional } & $<50$ & 45 & 58 \\
\hline & & $50+$ & 68 & 118 \\
\hline & \multirow{2}{*}{ Administrative Support } & $<50$ & 29 & 59 \\
\hline & & $50+$ & 68 & 118 \\
\hline & \multirow{2}{*}{ Technical Support } & $<50$ & 86 & 462 \\
\hline & & $50+$ & 110 & 306 \\
\hline & \multirow{2}{*}{ Service } & $<50$ & 38 & 286 \\
\hline & & $50+$ & 125 & 207 \\
\hline & \multirow{2}{*}{ Security and Fire } & $<50$ & 80 & 0 \\
\hline & & $50+$ & 0 & 500 \\
\hline & \multirow{2}{*}{ Crafts } & $<50$ & 115 & 0 \\
\hline & & $50+$ & 167 & 333 \\
\hline & \multirow{2}{*}{ Line Operators } & $<50$ & 71 & 143 \\
\hline & & $50+$ & 138 & 158 \\
\hline
\end{tabular}

Figure 12. Rates for Selected Diagnostic Categories by Job Category, Gender, and Age

\begin{tabular}{|c|c|c|c|c|}
\hline \multirow{2}{*}{ Cancer } & \multicolumn{4}{|c|}{ Rate per 1,000} \\
\hline & Job Category & Age & Men & Women \\
\hline & Profeccionol & $<50$ & 0 & 0 \\
\hline & 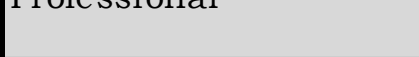 & $50+$ & 7 & 0 \\
\hline & Administrative Sunnort & $<50$ & 10 & 0 \\
\hline & A & $50+$ & 15 & 0 \\
\hline & Technicol Sunnort & $<50$ & 25 & $\mathbf{0}$ \\
\hline & 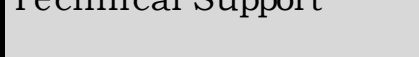 & $50+$ & 12 & 20 \\
\hline & Service & $<50$ & 0 & 0 \\
\hline & Netivice & $50+$ & 0 & 0 \\
\hline & Security and Fire & $<50$ & 0 & 0 \\
\hline & Necunity allu rile & $50+$ & 0 & 0 \\
\hline & Crafts & $<50$ & 0 & 0 \\
\hline & & $50+$ & 0 & 0 \\
\hline & Line Operators & $<50$ & 12 & 0 \\
\hline & 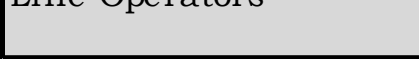 & $50+$ & 15 & 0 \\
\hline
\end{tabular}


Figure 12. Rates for Selected Diagnostic Categories by Job Category, Gender, and Age (Continued)

\begin{tabular}{|c|c|c|c|c|}
\hline \multirow{2}{*}{ Heart/Circulatory } & \multicolumn{4}{|c|}{ Rate per 1,000} \\
\hline & Job Category & Age & Men & Women \\
\hline & \multirow{2}{*}{ Professional } & $<50$ & 3 & 10 \\
\hline & & $50+$ & 5 & 20 \\
\hline & \multirow{2}{*}{ Administrative Support } & $<50$ & $\mathbf{0}$ & $\mathbf{0}$ \\
\hline & & $50+$ & 8 & 0 \\
\hline & \multirow{2}{*}{ Technical Support } & $<50$ & 0 & 0 \\
\hline & & $50+$ & 23 & 41 \\
\hline & \multirow{2}{*}{ Service } & $<50$ & 0 & 0 \\
\hline & & $50+$ & $\mathbf{0}$ & 0 \\
\hline & \multirow{2}{*}{ Security and Fire } & $<50$ & 40 & 0 \\
\hline & & $50+$ & 0 & 0 \\
\hline & \multirow{2}{*}{ Crafts } & $<50$ & 0 & 0 \\
\hline & & $50+$ & 30 & 0 \\
\hline & \multirow{2}{*}{ Line Operators } & $<50$ & 0 & 0 \\
\hline & & $50+$ & 15 & 0 \\
\hline
\end{tabular}

\begin{tabular}{|c|c|c|c|c|}
\hline \multirow{2}{*}{ Respiratory } & \multicolumn{4}{|c|}{ Rate per 1,000} \\
\hline & Job Category & Age & Men & Women \\
\hline & \multirow{2}{*}{ Professional } & $<50$ & 8 & 0 \\
\hline & & $50+$ & 0 & 0 \\
\hline & \multirow{2}{*}{ Administrative Support } & $<50$ & 0 & 0 \\
\hline & & $50+$ & 0 & 14 \\
\hline & \multirow{2}{*}{ Technical Support } & $<50$ & 12 & 0 \\
\hline & & $50+$ & 6 & 0 \\
\hline & \multirow{2}{*}{ Service } & $<50$ & 0 & 0 \\
\hline & & $50+$ & 0 & 34 \\
\hline & \multirow{2}{*}{ Security and Fire } & $<50$ & 0 & 0 \\
\hline & & $50+$ & 0 & 0 \\
\hline & \multirow{2}{*}{ Crafts } & $<50$ & 0 & 0 \\
\hline & & $50+$ & 4 & 111 \\
\hline & \multirow{2}{*}{ Line Operators } & $<50$ & 0 & 0 \\
\hline & & $50+$ & 15 & 0 \\
\hline
\end{tabular}


Figure 12. Rates for Selected Diagnostic Categories by Job Category, Gender, and Age (Continued)

\begin{tabular}{|c|c|c|c|c|}
\hline \multirow{2}{*}{ Injury } & \multicolumn{4}{|c|}{ Rate per 1,000 } \\
\hline & Job Category & Age & Men & Women \\
\hline & \multirow{2}{*}{ Professional } & $<50$ & 15 & 0 \\
\hline & & $50+$ & 11 & 20 \\
\hline & \multirow{2}{*}{ Administrative Support } & $<50$ & 0 & 10 \\
\hline & & $50+$ & 0 & 28 \\
\hline & \multirow{2}{*}{ Technical Support } & $<50$ & 0 & 77 \\
\hline & & $50+$ & 12 & 0 \\
\hline & \multirow{2}{*}{ Service } & $<50$ & 0 & 0 \\
\hline & & $50+$ & 31 & 0 \\
\hline & \multirow{2}{*}{ Security and Fire } & $<50$ & 40 & 0 \\
\hline & & $50+$ & 0 & 250 \\
\hline & \multirow{2}{*}{ Crafts } & $<50$ & 0 & 0 \\
\hline & & $50+$ & 34 & 0 \\
\hline & \multirow{2}{*}{ Line Operators } & $<50$ & 36 & 0 \\
\hline & & $50+$ & 31 & 13 \\
\hline
\end{tabular}

\section{Time Trends}

Figure 13. Age-Adjusted Rates for All Diagnoses Combined Among Women and Men from 2006 to 2010*

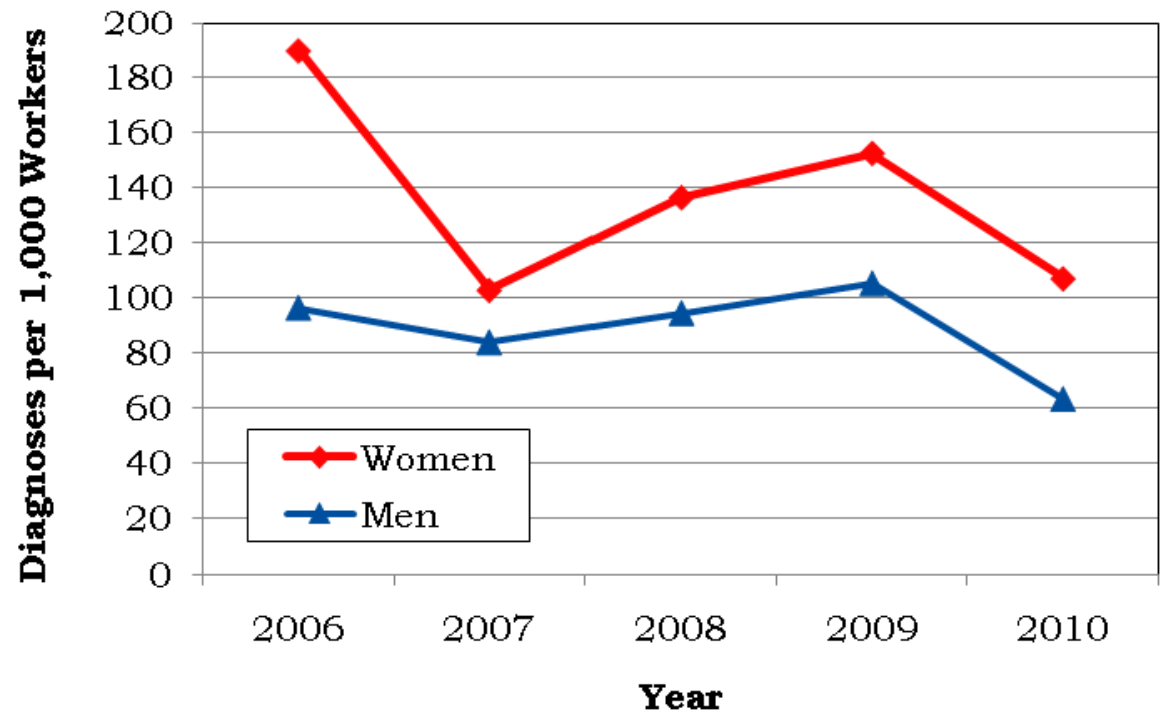

*Standardized to age distribution of 2000 U.S. population. 
Figure 14. Age-Adjusted Rates for Selected Diagnostic Categories Among Women and Men from 2006 to 2010*

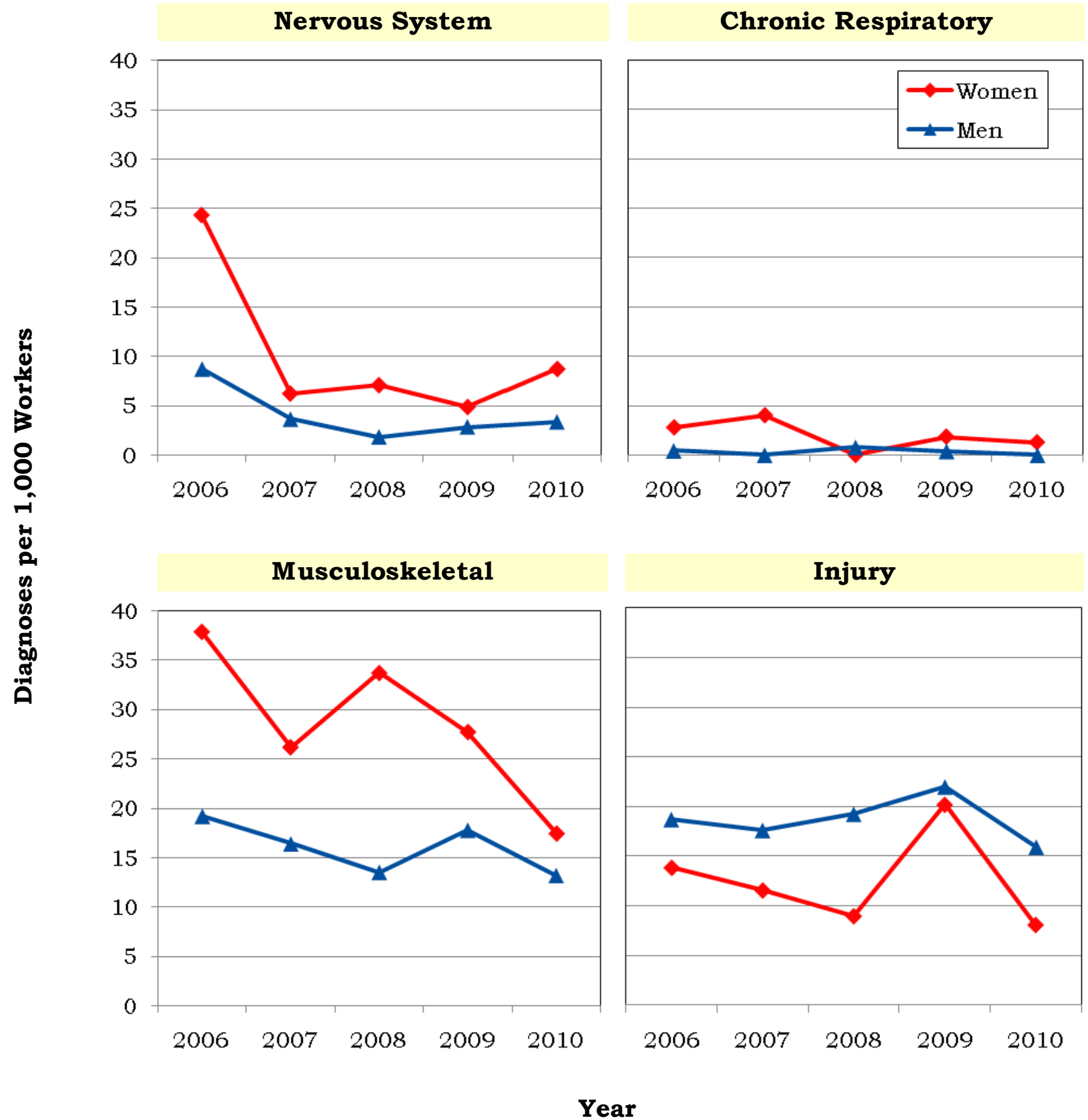

*Standardized to age distribution of 2000 U.S. population. 
Figure 15. Age-Adjusted Rates for All Diagnoses Combined Among Women and Men by Job Category from 2006 to 2010*

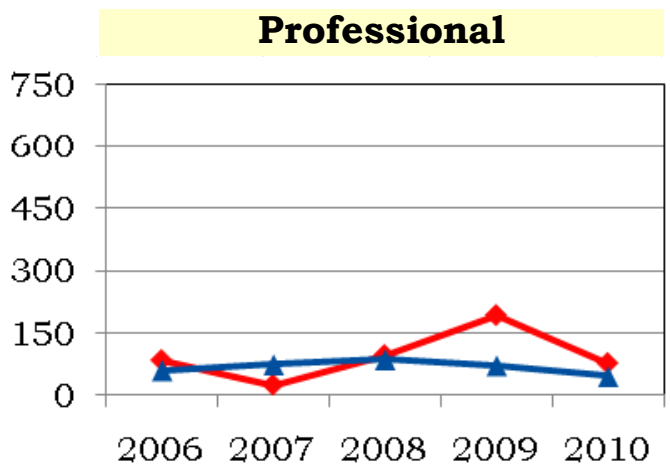

Administrative Support
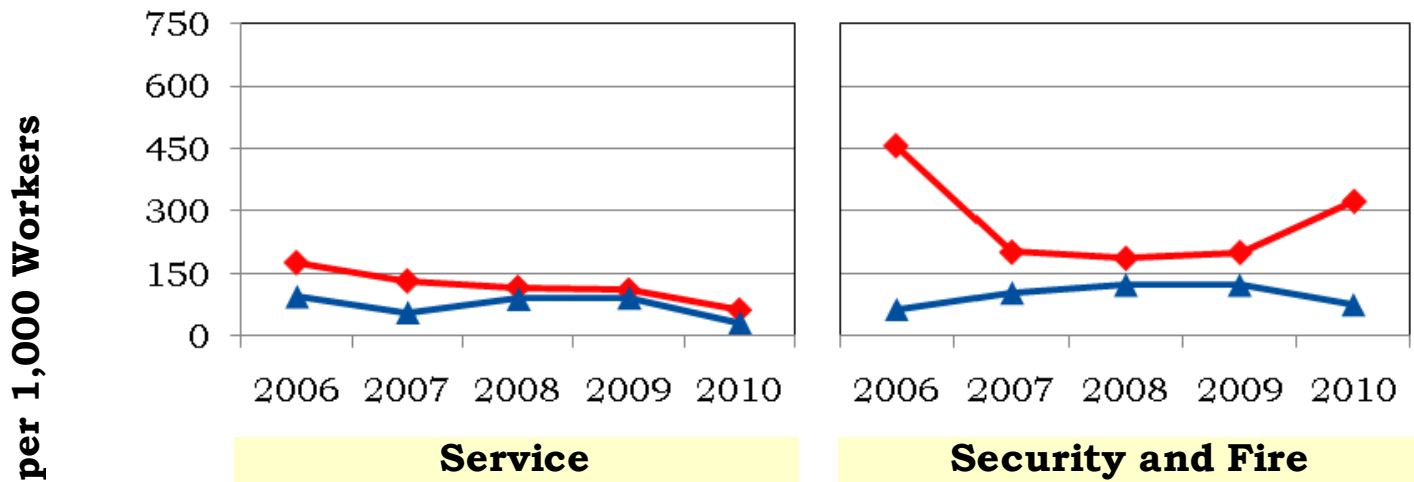

Technical Support

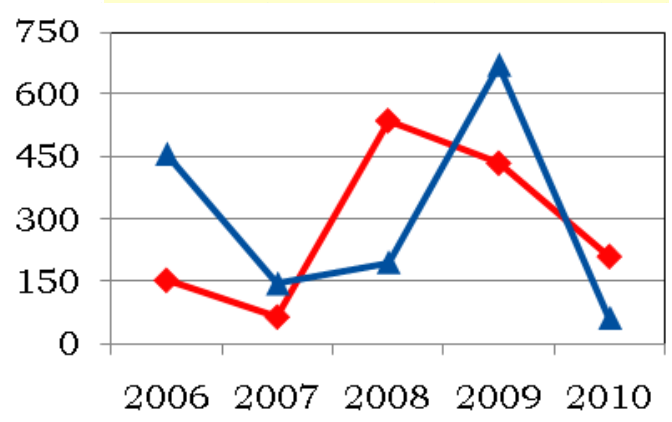

Crafts

20062007200820092010

Security and Fire

ญ

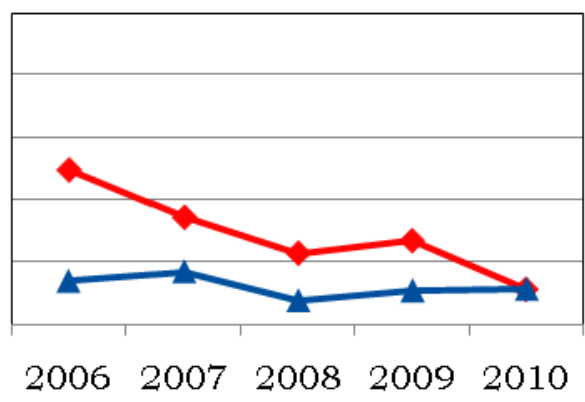

Line Operators
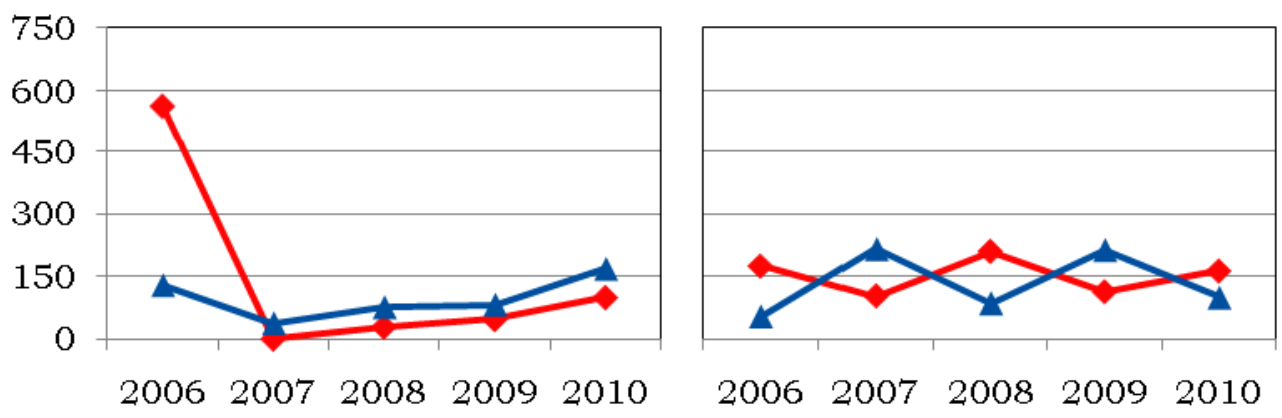

Year

*Standardized to age distribution of 2000 U.S. population. 


\section{Sentinel Health Events for Occupations (SHEOs)}

An occupational sentinel health event (SHEO) is a disease, disability, or death that is likely to be occupationally related. Although sentinel health events may indicate an occupational exposure, many may result from nonoccupational exposures. Sentinel health events are therefore assessed in two categories:

Definite Sentinel Health Events: Diseases that are unlikely to occur in the absence of an occupational exposure (e.g., asbestosis).

Possible Sentinel Health Events: Diseases that may be occupational but can also occur in the absence of an occupational exposure (e.g., lung cancer or carpal tunnel syndrome).

Figure 16. Characteristics of SHEOs by Gender

\begin{tabular}{|l|c|c|c|c|}
\hline \multirow{2}{*}{} & \multicolumn{2}{|c|}{$\begin{array}{c}\text { Total Number of } \\
\text { SHEO Diagnoses }\end{array}$} & \multicolumn{2}{c|}{$\begin{array}{c}\text { Total Number of } \\
\text { Days Absent }\end{array}$} \\
\cline { 2 - 5 } & Men & Women & Men & W omen \\
\hline Definite & 2 & 0 & 12 & 0 \\
\hline Possible & 1 & 2 & 97 & 78 \\
\hline Total & 3 & 2 & 109 & 78 \\
\hline
\end{tabular}

Figure 17. SHEO Diagnoses by Gender

\begin{tabular}{|l|c|c|}
\hline \multirow{2}{*}{ Diagnoses } & \multicolumn{2}{c|}{ Gender } \\
\cline { 2 - 3 } & W omen & M en \\
\hline Carpal Tunnel Syndrome & 2 & 1 \\
\hline Injuries & 0 & 1 \\
\hline Other Conditions & 0 & 1 \\
\hline
\end{tabular}


Occupational Safety and Health Administration (OSHA)-Recordable Events

Figure 18. OSHA-Recordable Events by Gender and Age

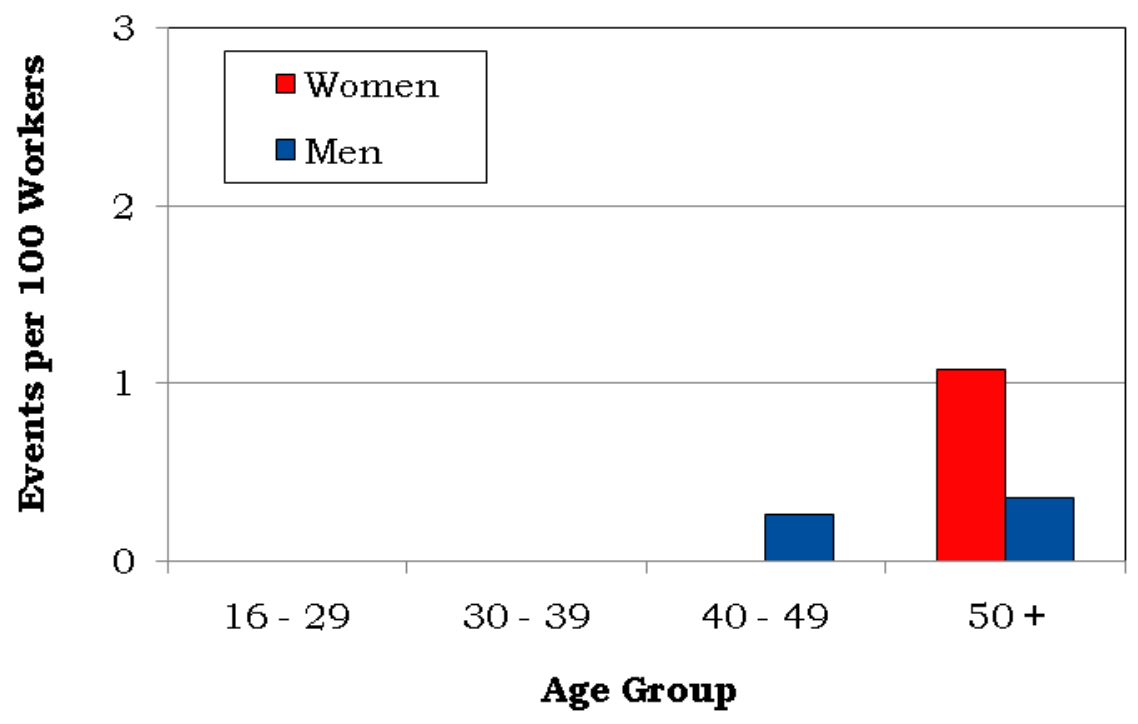

Figure 19. OSHA-Recordable Events by Job Category and Gender

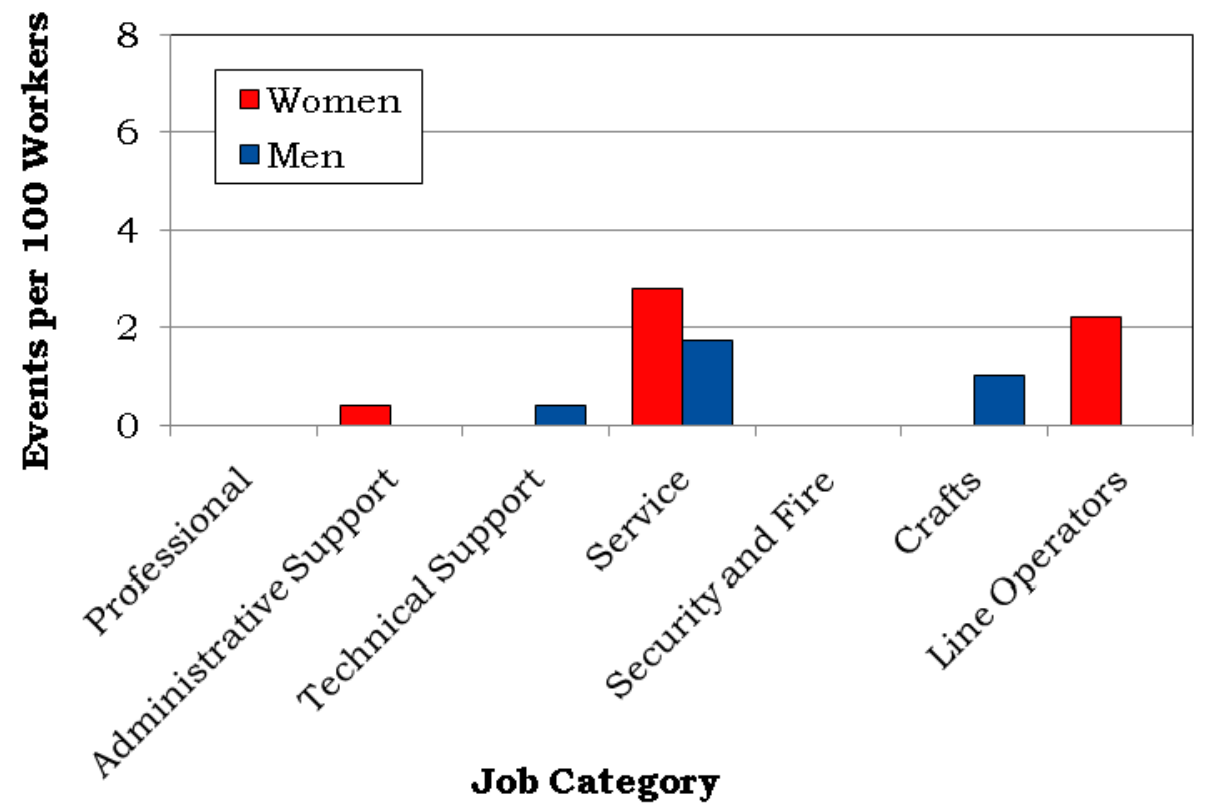




\section{Diagnostic and Accident Categories for OSHA-Recordable Events}

Figure 20. OSHA-Recordable Diagnoses by Diagnostic Category and Gender

\begin{tabular}{|l|c|c|}
\hline \multirow{2}{*}{ Diagnostic Category } & \multicolumn{2}{c|}{ Gender } \\
\cline { 2 - 3 } & Women & M en \\
\hline Genitourinary & $\mathbf{1}$ & $\mathbf{0}$ \\
\hline Musculoskeletal & $\mathbf{4}$ & $\mathbf{3}$ \\
\hline Respiratory & $\mathbf{1}$ & $\mathbf{0}$ \\
\hline Unspecified Symptoms & $\mathbf{2}$ & $\mathbf{1}$ \\
\hline Injury & $\mathbf{5}$ & $\mathbf{4}$ \\
\hline Fractures - Upper Limb & $\mathbf{1}$ & $\mathbf{0}$ \\
\hline Fractures - Lower Limb & $\mathbf{1}$ & $\mathbf{0}$ \\
\hline Other Sprains \& Strains & $\mathbf{0}$ & $\mathbf{1}$ \\
\hline Open Wounds - Head, Neck, Trunk & $\mathbf{0}$ & $\mathbf{1}$ \\
\hline Open Wounds - Upper Limb & $\mathbf{0}$ & $\mathbf{2}$ \\
\hline Superficial Injuries & $\mathbf{3}$ & $\mathbf{0}$ \\
\hline
\end{tabular}

Figure 21. OSHA-Recordable Accidents by Type and Gender

\begin{tabular}{|l|c|c|}
\hline \multirow{2}{*}{ Accident Category } & \multicolumn{2}{|c|}{ Gender } \\
\cline { 2 - 3 } & Women & Men \\
\cline { 2 - 3 } & $\begin{array}{c}\text { Number of } \\
\text { Accidents }\end{array}$ & $\begin{array}{c}\text { Number of } \\
\text { Accidents }\end{array}$ \\
\hline Falls & $\mathbf{3}$ & $\mathbf{1}$ \\
\hline Other Accidents & $\mathbf{1}$ & $\mathbf{4}$ \\
\hline Struck by an Object & $\mathbf{0}$ & $\mathbf{2}$ \\
\hline Caught Between Objects & $\mathbf{0}$ & $\mathbf{1}$ \\
\hline Machinery & $\mathbf{0}$ & $\mathbf{1}$ \\
\hline Repetitive Trauma & $\mathbf{1}$ & $\mathbf{0}$ \\
\hline Total & $\mathbf{4}$ & $\mathbf{5}$ \\
\hline
\end{tabular}




\section{Rates of OSHA-Recordable Events}

Figure 22. OSHA-Recordable Rates by Age and Job Categories Among Women, All Diagnoses Combined

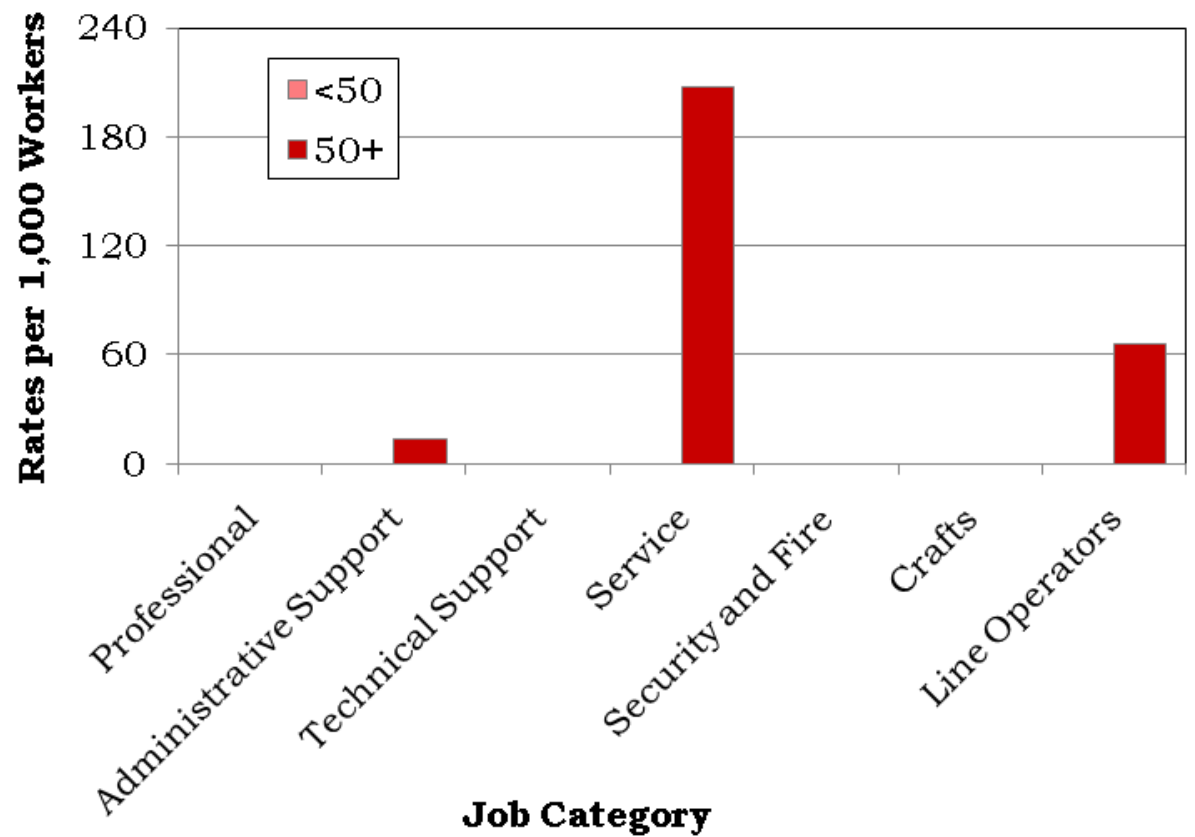

Figure 23. OSHA-Recordable Rates by Age and Job Categories Among Men, All Diagnoses Combined

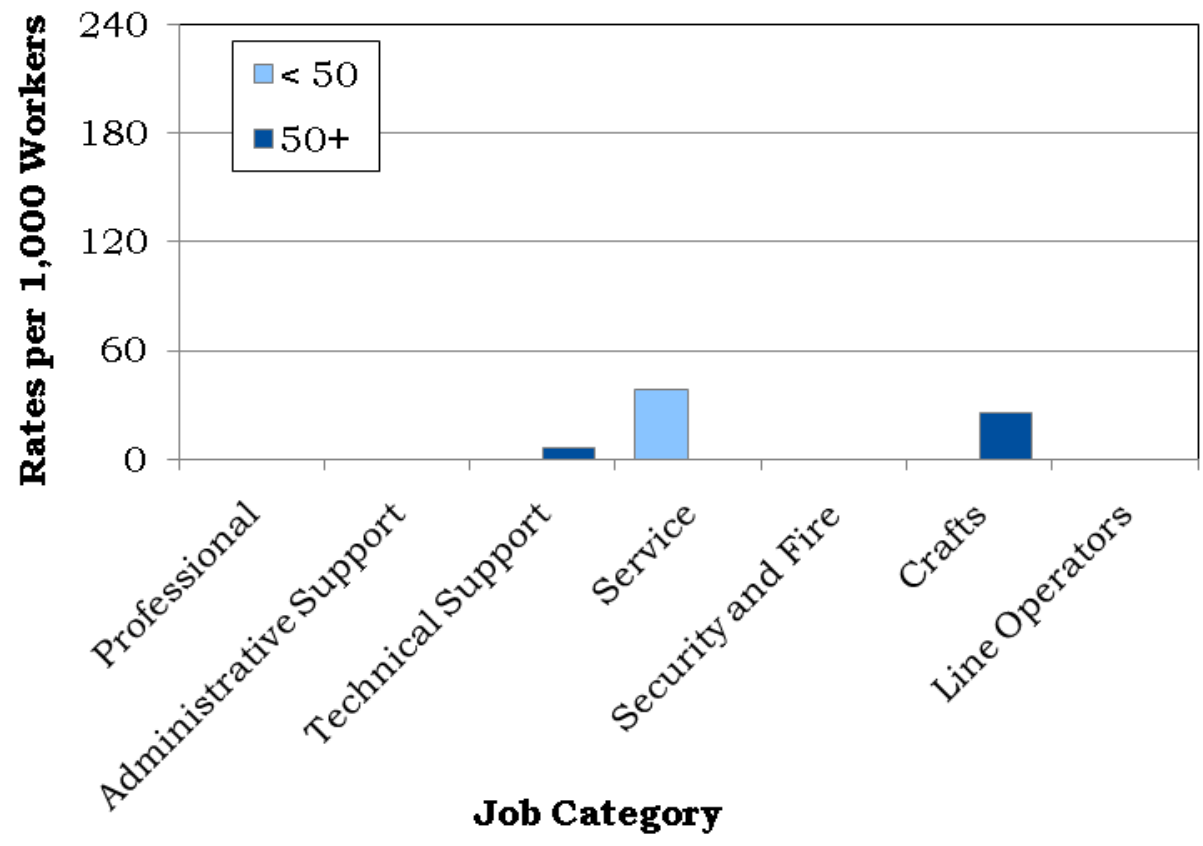




\section{Time Trends for OSHA-Recordable Events}

Figure 24. Age-Adjusted Rates for All OSHA-Recordable Diagnoses Combined Among Women and Men by Job Category from 2006 to 2010*

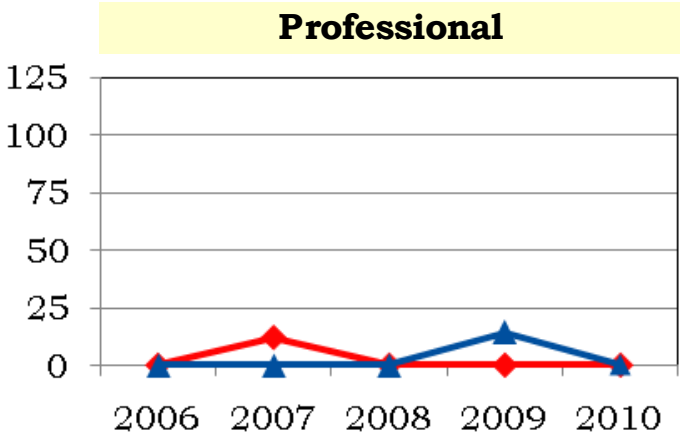

Administrative Sup port
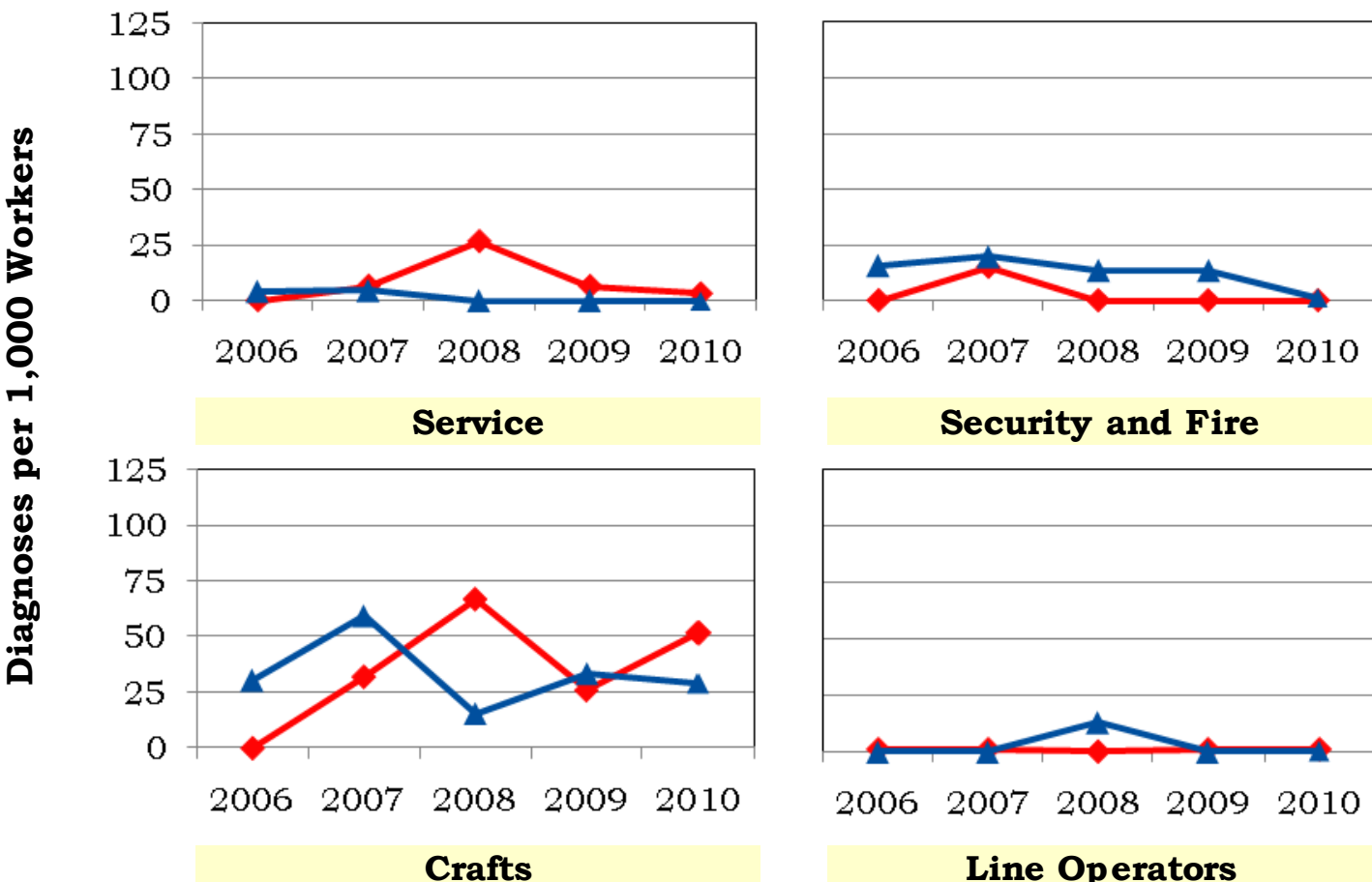

Security and Fire
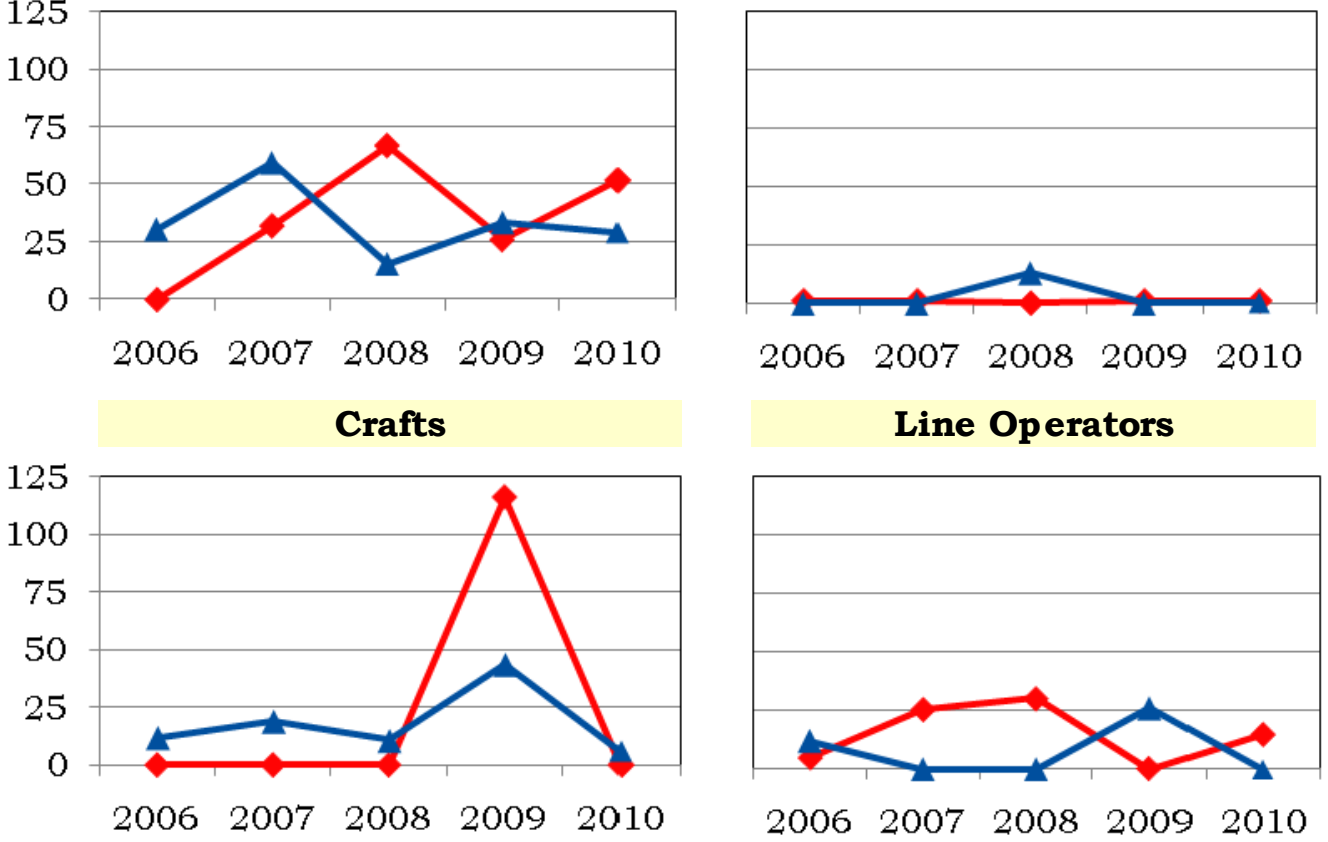

Year

*Standardized to age distribution of 2000 U.S. population. 
Appendices 
Kansas City Plant 2010

Absence Data

Appendix A. Work Force by Gender, Age, and Job Category

\begin{tabular}{|c|c|c|c|c|c|c|c|c|c|c|c|}
\hline \multirow{3}{*}{ Job Category } & \multicolumn{5}{|c|}{ Women } & \multicolumn{5}{|c|}{ Men } & \multirow[b]{3}{*}{ TOTAL } \\
\hline & \multicolumn{4}{|c|}{ Age Group } & \multirow[b]{2}{*}{ TOTAL } & \multicolumn{4}{|c|}{ Age Group } & \multirow[b]{2}{*}{ TOTAL } & \\
\hline & $16-29$ & $30-39$ & $40-49$ & $50+$ & & $16-29$ & $30-39$ & $40-49$ & $50+$ & & \\
\hline Professional & 41 & 19 & 44 & 51 & 155 & 86 & 117 & 196 & 440 & 839 & 994 \\
\hline Administrative Support & 13 & 28 & 60 & 144 & 245 & 17 & 35 & 53 & 132 & 237 & 482 \\
\hline Technical Support & 4 & 2 & 7 & 49 & 62 & 14 & 25 & 42 & 172 & 253 & 315 \\
\hline Service & 3 & 1 & 3 & 29 & 36 & 15 & 3 & 8 & 32 & 58 & 94 \\
\hline Security and Fire & 1 & 0 & 1 & 4 & 6 & 4 & 10 & 11 & 34 & 59 & 65 \\
\hline Crafts & 0 & 0 & 0 & 18 & 18 & 3 & 18 & 40 & 233 & 294 & 312 \\
\hline Line Operators & 4 & 3 & 7 & 76 & 90 & 30 & 23 & 31 & 65 & 149 & 239 \\
\hline TOTAL & 66 & 53 & 122 & 371 & 612 & 169 & 231 & 381 & 1,108 & 1,889 & 2,501 \\
\hline
\end{tabular}

Appendix B. Age Distribution of the Work Force by Gender

\begin{tabular}{|l|r|r|r|r|r|r|r|r|}
\hline \multirow{3}{*}{ Year } & \multicolumn{4}{|c|}{ Women } & \multicolumn{4}{c|}{ Men } \\
\cline { 2 - 9 } & \multicolumn{2}{|c|}{ Percent Distribution by Age Group } & \multicolumn{1}{c|}{ Percent Distribution by Age Group } \\
\cline { 2 - 9 } & $\mathbf{1 6}-\mathbf{2 9}$ & $\mathbf{3 0}-\mathbf{3 9}$ & $\mathbf{4 0}-\mathbf{4 9}$ & $\mathbf{5 0}+$ & $\mathbf{1 6}-\mathbf{2 9}$ & $\mathbf{3 0}-\mathbf{3 9}$ & $\mathbf{4 0}-\mathbf{4 9}$ & $\mathbf{5 0}+$ \\
\hline $\mathbf{2 0 0 2}$ & 9.74 & 9.62 & 40.68 & 39.95 & 8.47 & 8.60 & 42.40 & 40.53 \\
\hline $\mathbf{2 0 0 3}$ & 6.88 & 9.04 & 40.76 & 43.31 & 8.02 & 7.46 & 42.54 & 41.98 \\
\hline $\mathbf{2 0 0 4}$ & 10.52 & 7.79 & 37.98 & 43.72 & 9.04 & 7.25 & 40.96 & 42.75 \\
\hline $\mathbf{2 0 0 5}$ & 9.86 & 7.46 & 35.63 & 47.04 & 8.51 & 7.66 & 37.94 & 45.88 \\
\hline $\mathbf{2 0 0 6}$ & 9.23 & 6.85 & 33.78 & 50.15 & 8.31 & 7.81 & 34.78 & 49.10 \\
\hline $\mathbf{2 0 0 7}$ & 9.57 & 7.10 & 30.71 & 52.62 & 9.43 & 8.31 & 31.12 & 51.14 \\
\hline $\mathbf{2 0 0 8}$ & 8.90 & 8.13 & 28.37 & 54.60 & 9.56 & 9.96 & 27.59 & 52.88 \\
\hline $\mathbf{2 0 0 9}$ & 8.61 & 8.45 & 24.40 & 58.53 & 8.46 & 10.73 & 23.94 & 56.86 \\
\hline $\mathbf{2 0 1 0}$ & 10.78 & 8.66 & 19.93 & 60.62 & 8.95 & 12.23 & 20.17 & 58.66 \\
\hline
\end{tabular}


Kansas City Plant 2010

Absence Data

Appendix C. Total Number of Workers Who Reported at Least One Absence by Gender, Age, and Job Category*

\begin{tabular}{|c|c|c|c|c|c|c|c|c|c|c|c|}
\hline \multirow{3}{*}{ Job Category } & \multicolumn{5}{|c|}{ Women } & \multicolumn{5}{|c|}{ Men } & \multirow[b]{3}{*}{ TOTAL } \\
\hline & \multicolumn{4}{|c|}{ Age Group } & \multirow[b]{2}{*}{ TOTAL } & \multicolumn{4}{|c|}{ Age Group } & \multirow[b]{2}{*}{ TOTAL } & \\
\hline & $16-29$ & 30 - 39 & $40-49$ & $50+$ & & 16 - 29 & 30 - 39 & $40-49$ & $50+$ & & \\
\hline Professional & 1 & 1 & 3 & 4 & 9 & 2 & 3 & 9 & 21 & 35 & 44 \\
\hline Administrative Support & 0 & 1 & 3 & 11 & 15 & 0 & 0 & 2 & 7 & 9 & 24 \\
\hline Technical Support & 1 & 0 & 1 & 6 & 8 & 0 & 1 & 5 & 14 & 20 & 28 \\
\hline Service & 0 & 0 & 1 & 5 & 6 & 0 & 0 & 1 & 2 & 3 & 9 \\
\hline Security and Fire & 0 & 0 & 0 & 2 & 2 & 1 & 0 & 1 & 0 & 2 & 4 \\
\hline Crafts & 0 & 0 & 0 & 4 & 4 & 1 & 0 & 3 & 33 & 37 & 41 \\
\hline Line Operators & 1 & 0 & 0 & 8 & 9 & 1 & 0 & 3 & 7 & 11 & 20 \\
\hline TOTAL & 3 & 2 & 8 & 40 & 53 & 5 & 4 & 24 & 84 & 117 & 170 \\
\hline
\end{tabular}

*Only those job categories and gender/age combinations with at least one absence appear in this table.

Appendix D. Total Number of Absences by Gender, Age, and Job Category*

\begin{tabular}{|c|c|c|c|c|c|c|c|c|c|c|c|}
\hline \multirow{3}{*}{ Job Category } & \multicolumn{5}{|c|}{ Women } & \multicolumn{5}{|c|}{ Men } & \multirow[b]{3}{*}{ TOTAL } \\
\hline & \multicolumn{4}{|c|}{ Age Group } & \multirow[b]{2}{*}{ TOTAL } & \multicolumn{4}{|c|}{ Age Group } & \multirow[b]{2}{*}{ TOTAL } & \\
\hline & $16-29$ & 30 - 39 & $40-49$ & $50+$ & & $16-29$ & 30 - 39 & $40-49$ & $50+$ & & \\
\hline Professional & 1 & 1 & 3 & 5 & 10 & 2 & 3 & 10 & 23 & 38 & 48 \\
\hline Administrative Support & 0 & 1 & 3 & 11 & 15 & 0 & 0 & 2 & 8 & 10 & 25 \\
\hline Technical Support & 1 & 0 & 4 & 7 & 12 & 0 & 1 & 6 & 17 & 24 & 36 \\
\hline Service & 0 & 0 & 2 & 5 & 7 & 0 & 0 & 1 & 2 & 3 & 10 \\
\hline Security and Fire & 0 & 0 & 0 & 2 & 2 & 1 & 0 & 1 & 0 & 2 & 4 \\
\hline Crafts & 0 & 0 & 0 & 5 & 5 & 1 & 0 & 4 & 36 & 41 & 46 \\
\hline Line Operators & 1 & 0 & 0 & 9 & 10 & 1 & 0 & 3 & 8 & 12 & 22 \\
\hline TOTAL & 3 & 2 & 12 & 44 & 61 & 5 & 4 & 27 & 94 & 130 & 191 \\
\hline
\end{tabular}

*Only those job categories and gender/age combinations with at least one absence appear in this table. 
Kansas City Plant 2010

Absence Data

Appendix E. Distribution of the Number of Calendar Days Missed per Absence by Gender and Age*

\begin{tabular}{|c|c|c|c|c|c|c|c|c|c|c|c|}
\hline \multirow{3}{*}{$\begin{array}{c}\text { Number of } \\
\text { Calendar Days }\end{array}$} & \multicolumn{5}{|c|}{ Women } & \multicolumn{5}{|c|}{ Men } & \multirow[b]{3}{*}{ TOTAL } \\
\hline & \multicolumn{4}{|c|}{ Age Group } & \multirow[b]{2}{*}{ TOTAL } & \multicolumn{4}{|c|}{ Age Group } & \multirow[b]{2}{*}{ TOTAL } & \\
\hline & $16-29$ & $30-39$ & $40-49$ & $50+$ & & $16-29$ & 30 - 39 & $40-49$ & $50+$ & & \\
\hline$<15$ & 2 & 1 & 7 & 20 & 30 & 2 & 1 & 15 & 25 & 43 & 73 \\
\hline $15-28$ & 0 & 1 & 1 & 11 & 13 & 2 & 2 & 6 & 22 & 32 & 45 \\
\hline $29-42$ & 0 & 0 & 2 & 6 & 8 & 0 & 1 & 1 & 19 & 21 & 29 \\
\hline $43-56$ & 1 & 0 & 1 & 6 & 8 & 0 & 0 & 1 & 8 & 9 & 17 \\
\hline $57-91$ & 0 & 0 & 1 & 1 & 2 & 1 & 0 & 3 & 15 & 19 & 21 \\
\hline $92-182$ & 0 & 0 & 0 & 0 & 0 & 0 & 0 & 1 & 4 & 5 & 5 \\
\hline $183+$ & 0 & 0 & 0 & 0 & 0 & 0 & 0 & 0 & 1 & 1 & 1 \\
\hline TOTAL & 3 & 2 & 12 & 44 & 61 & 5 & 4 & 27 & 94 & 130 & 191 \\
\hline
\end{tabular}

*Only those gender/age combinations with at least one absence appear in this table. 
Kansas City Plant 2010

Absence Data

Appendix F. Distribution of the Number of Calendar Days Missed per Absence by Gender and Job Category*

Women

\begin{tabular}{|c|c|c|c|c|c|c|c|c|}
\hline \multirow{2}{*}{$\begin{array}{c}\text { Number of } \\
\text { Calendar Days }\end{array}$} & \multicolumn{7}{|c|}{ Job Category } & \multirow[b]{2}{*}{ TOTAL } \\
\hline & Professional & $\begin{array}{c}\text { Administrative } \\
\text { Support }\end{array}$ & Technical Support & Service & Security and Fire & Crafts & Line Operators & \\
\hline$<15$ & 6 & 7 & 6 & 4 & 1 & 1 & 5 & 30 \\
\hline $15-28$ & 2 & 4 & 4 & 0 & 1 & 1 & 1 & 13 \\
\hline $57-91$ & 1 & 0 & 0 & 1 & 0 & 0 & 0 & 2 \\
\hline $92-182$ & 0 & 0 & 0 & 0 & 0 & 0 & 0 & 0 \\
\hline $183+$ & 0 & 0 & 0 & 0 & 0 & 0 & 0 & 0 \\
\hline TOTAL & 10 & 15 & 12 & 7 & 2 & 5 & 10 & 61 \\
\hline
\end{tabular}

Men

\begin{tabular}{|c|c|c|c|c|c|c|c|c|}
\hline \multirow{2}{*}{$\begin{array}{l}\text { Number of } \\
\text { Calendar Days }\end{array}$} & \multicolumn{7}{|c|}{ Job Category } & \multirow[b]{2}{*}{ TOTAL } \\
\hline & Professional & $\begin{array}{c}\text { Administrative } \\
\text { Support }\end{array}$ & Technical Support & Service & Security and Fire & Crafts & Line Operators & \\
\hline$<15$ & 16 & 3 & 10 & 1 & 0 & 9 & 4 & 43 \\
\hline $15-28$ & 12 & 2 & 6 & 1 & 1 & 10 & 0 & 32 \\
\hline $57-91$ & 3 & 3 & 2 & 0 & 1 & 8 & 2 & 19 \\
\hline $92-182$ & 1 & 0 & 1 & 1 & 0 & 0 & 2 & 5 \\
\hline $183+$ & 0 & 0 & 0 & 0 & 0 & 1 & 0 & 1 \\
\hline TOTAL & 38 & 10 & 24 & 3 & 2 & 41 & 12 & 130 \\
\hline
\end{tabular}

*Only those gender/job category combinations with at least one absence appear in this table. 


\section{Kansas City Plant 2010}

Absence Data

Appendix G. Number of Diagnoses in Each Diagnostic Category by Gender and Age*

\begin{tabular}{|c|c|c|c|c|c|c|}
\hline & & \multicolumn{5}{|c|}{ Women } \\
\hline & & \multicolumn{4}{|c|}{ Age Group } & \multirow[b]{2}{*}{ TOTAL } \\
\hline & & $16-29$ & $30-39$ & $40-49$ & $50+$ & \\
\hline Diagnostic Category & ICD-9-CM Code & \multirow[b]{2}{*}{0} & \multirow[b]{2}{*}{1} & \multirow[b]{2}{*}{0} & \multirow[b]{2}{*}{0} & \multirow[b]{2}{*}{1} \\
\hline INFECTIOUS \& PARASITIC DISEASES (DIS) & 001-139 & & & & & \\
\hline -Other Bacterial Dis & $030-041$ & 0 & 1 & 0 & 0 & 1 \\
\hline MALIGNANT NEOPLASMS & $\begin{array}{l}140-208 \\
209.0-209.3 \\
230-234\end{array}$ & 0 & 0 & 0 & 1 & 1 \\
\hline -Lip, Oral Cavity, Pharynx & $140-149$ & 0 & 0 & 0 & 0 & 0 \\
\hline -Bone, Connective Tissue, Skin & $170-173,176$ & 0 & 0 & 0 & 0 & 0 \\
\hline -Breast & 174-175 & 0 & 0 & 0 & 1 & 1 \\
\hline -Genitourinary & 179-189 & 0 & 0 & 0 & 0 & 0 \\
\hline -Other \& Unspecified Sites & 195-199, 209.7 & 0 & 0 & 0 & 0 & 0 \\
\hline -Lymphatic \& Hematopoietic & $200-208$ & 0 & 0 & 0 & 0 & 0 \\
\hline BENIGN \& UNCERTAIN NEOPLASMS & $\begin{array}{l}209.4-209.6 \\
210-229,235-239\end{array}$ & 0 & 0 & 0 & 2 & 2 \\
\hline ENDOCRINE/METABOLIC/IMMUNITY & $240-279$ & 0 & 0 & 0 & 4 & 4 \\
\hline -Thyroid Gland Disorders & $240-246$ & 0 & 0 & 0 & 2 & 2 \\
\hline -Other Endocrine Gland Dis & $249-259$ & 0 & 0 & 0 & 1 & 1 \\
\hline -Other Metabolic \& Immunity Disorders & $270-279$ & 0 & 0 & 0 & 1 & 1 \\
\hline BLOOD \& BLOOD-FORMING ORGANS & 280-289 & 0 & 0 & 0 & 1 & 1 \\
\hline MENTAL DISORDERS & $290-319$ & 2 & 0 & 4 & 0 & 6 \\
\hline -Psychoses & 290-299 & 1 & 0 & 0 & 0 & 1 \\
\hline -Non-Psychotic Disorders & $300-302,306-316$ & 1 & 0 & 4 & 0 & 5 \\
\hline NERVOUS SYSTEM (NS) \& SENSE ORGANS & 320-389 & 0 & 1 & 1 & 4 & 6 \\
\hline -Inflammatory Dis of Central NS & $320-326$ & 0 & 0 & 1 & 0 & 1 \\
\hline -Other Disorders of Central NS & $340-349$ & 0 & 0 & 0 & 1 & 1 \\
\hline -Disorders of Peripheral NS & $350-359$ & 0 & 0 & 0 & 2 & 2 \\
\hline -Disorders of Eye & $360-379$ & 0 & 0 & 0 & 1 & 1 \\
\hline -Diseases of Ear \& Mastoid & $380-389$ & 0 & 1 & 0 & 0 & 1 \\
\hline CIRCULATORY SYSTEM & $390-459$ & 0 & 0 & 1 & 3 & 4 \\
\hline -Ischemic Heart Dis & $410-414$ & 0 & 0 & 0 & 3 & 3 \\
\hline -Other Heart Dis & $420-429$ & 0 & 0 & 0 & 0 & 0 \\
\hline -Cerebrovascular Dis & $430-438$ & 0 & 0 & 0 & 0 & 0 \\
\hline -Dis of Arteries \& Capillaries & $440-449$ & 0 & 0 & 0 & 0 & 0 \\
\hline -Dis of Veins, Lymphatics, Other & $451-459$ & 0 & 0 & 1 & 0 & 1 \\
\hline RESPIRATORY SYSTEM & $460-519$ & 0 & 0 & 0 & 5 & 5 \\
\hline -Other Dis Upper Respiratory Tract & $470-478$ & 0 & 0 & 0 & 2 & 2 \\
\hline -Pneumonia \& Influenza & $480-488$ & 0 & 0 & 0 & 1 & 1 \\
\hline -Chronic Obstructive Dis & \begin{tabular}{|l|}
$490-496$ \\
\end{tabular} & 0 & 0 & 0 & 1 & 1 \\
\hline -Other Respiratory Dis & $510-519$ & 0 & 0 & 0 & 1 & 1 \\
\hline
\end{tabular}

(Continued)

*Only those diagnostic categories and gender/age combinations with at least one occurrence appear in this table. 


\section{Kansas City Plant 2010}

Absence Data

Appendix G. Number of Diagnoses in Each Diagnostic Category by Gender and Age*

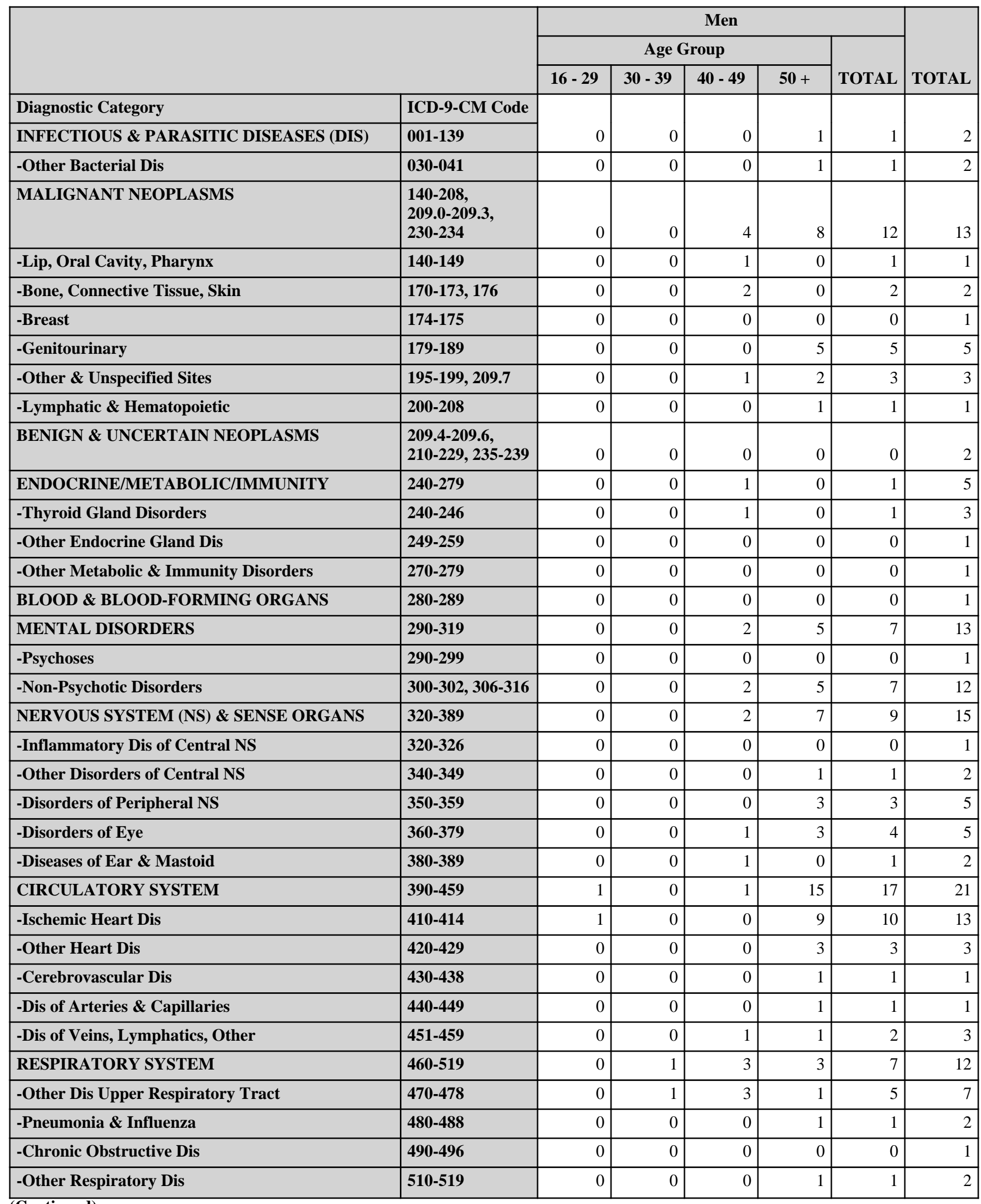

(Continued)

*Only those diagnostic categories and gender/age combinations with at least one occurrence appear in this table. 


\section{Kansas City Plant 2010}

Absence Data

Appendix G. Number of Diagnoses in Each Diagnostic Category by Gender and Age*

\begin{tabular}{|c|c|c|c|c|c|c|}
\hline & & \multicolumn{5}{|c|}{ Women } \\
\hline & & \multicolumn{4}{|c|}{ Age Group } & \multirow[b]{2}{*}{ TOTAL } \\
\hline & & $16-29$ & $30-39$ & $40-49$ & $50+$ & \\
\hline Diagnostic Category & ICD-9-CM Code & \multirow[b]{2}{*}{0} & \multirow[b]{2}{*}{0} & \multirow[b]{2}{*}{4} & \multirow[b]{2}{*}{7} & \multirow[b]{2}{*}{11} \\
\hline DIGESTIVE SYSTEM & $520-579$ & & & & & \\
\hline -Oral Cavity, Saliva Glands, Jaw & $520-529$ & 0 & 0 & 0 & 0 & 0 \\
\hline -Esophagus, Stomach, Duodenum & $530-538$ & 0 & 0 & 0 & 1 & 1 \\
\hline -Appendicitis & $540-543$ & 0 & 0 & 0 & 1 & 1 \\
\hline -Hernias & $550-553$ & 0 & 0 & 0 & 0 & 0 \\
\hline -Enteritis, Colitis & $555-558$ & 0 & 0 & 0 & 2 & 2 \\
\hline -Other Intestinal Dis & $560-569$ & 0 & 0 & 0 & 1 & 1 \\
\hline -Other Digestive Dis & $570-579$ & 0 & 0 & 4 & 2 & 6 \\
\hline GENITOURINARY SYSTEM & $580-629$ & 2 & 0 & 0 & 3 & 5 \\
\hline -Nephritis, Nephrosis & $580-589$ & 0 & 0 & 0 & 0 & 0 \\
\hline -Other Urinary Dis & $590-599$ & 0 & 0 & 0 & 2 & 2 \\
\hline -Male Genital Organ Dis & $600-608$ & 0 & 0 & 0 & 0 & 0 \\
\hline -Other Female Disorders & $617-629$ & 2 & 0 & 0 & 1 & 3 \\
\hline SKIN AND SUBCUTANEOUS TISSUE & $680-709$ & 0 & 1 & 0 & 1 & 2 \\
\hline -Infections & $680-686$ & 0 & 1 & 0 & 0 & 1 \\
\hline -Other & 700-709 & 0 & 0 & 0 & 1 & 1 \\
\hline $\begin{array}{l}\text { MUSCULOSKELETAL \& CONNECTIVE } \\
\text { TISSUE }\end{array}$ & 710-739 & 0 & 0 & 2 & 19 & 21 \\
\hline -Arthropathies & $710-719$ & 0 & 0 & 0 & 6 & 6 \\
\hline -Dorsopathies & $720-724$ & 0 & 0 & 0 & 6 & 6 \\
\hline -Rheumatism, Excluding Back & 725-729 & 0 & 0 & 2 & 6 & 8 \\
\hline -Other Dis \& Acquired Deformities & 730-739 & 0 & 0 & 0 & 1 & 1 \\
\hline $\begin{array}{l}\text { SYMPTOMS, SIGNS, \& ILL-DEFINED } \\
\text { CONDITIONS }\end{array}$ & 780-799 & 1 & 0 & 0 & 7 & 8 \\
\hline -Symptoms & $780-789$ & 1 & 0 & 0 & 5 & 6 \\
\hline -Non-Specific Abnormal Findings & $790-796$ & 0 & 0 & 0 & 2 & 2 \\
\hline INJURY \& POISONING & $800-999$ & 0 & 0 & 2 & 7 & 9 \\
\hline -Fracture - Neck, Trunk & 805-809 & 0 & 0 & 0 & 1 & 1 \\
\hline -Fracture - Upper Limb & $810-819$ & 0 & 0 & 0 & 1 & 1 \\
\hline -Fracture - Lower Limb & $820-829$ & 0 & 0 & 0 & 1 & 1 \\
\hline -Dislocation & $830-839$ & 0 & 0 & 0 & 0 & 0 \\
\hline -Sprains \& Strains - Back & 846-847 & 0 & 0 & 0 & 1 & 1 \\
\hline -Sprains \& Strains - Other & $840-845,848$ & 0 & 0 & 0 & 2 & 2 \\
\hline -Intracranial Injury & $850-854$ & 0 & 0 & 0 & 0 & 0 \\
\hline -Open Wound - Head, Neck, Trunk & $870-879$ & 0 & 0 & 0 & 0 & 0 \\
\hline -Burns & 940-949 & 0 & 0 & 0 & 0 & 0 \\
\hline -Complications \& Unspecified Injuries & 958-959 & 0 & 0 & 1 & 0 & 1 \\
\hline
\end{tabular}

(Continued)

*Only those diagnostic categories and gender/age combinations with at least one occurrence appear in this table. 


\section{Kansas City Plant 2010}

\section{Absence Data}

Appendix G. Number of Diagnoses in Each Diagnostic Category by Gender and Age*

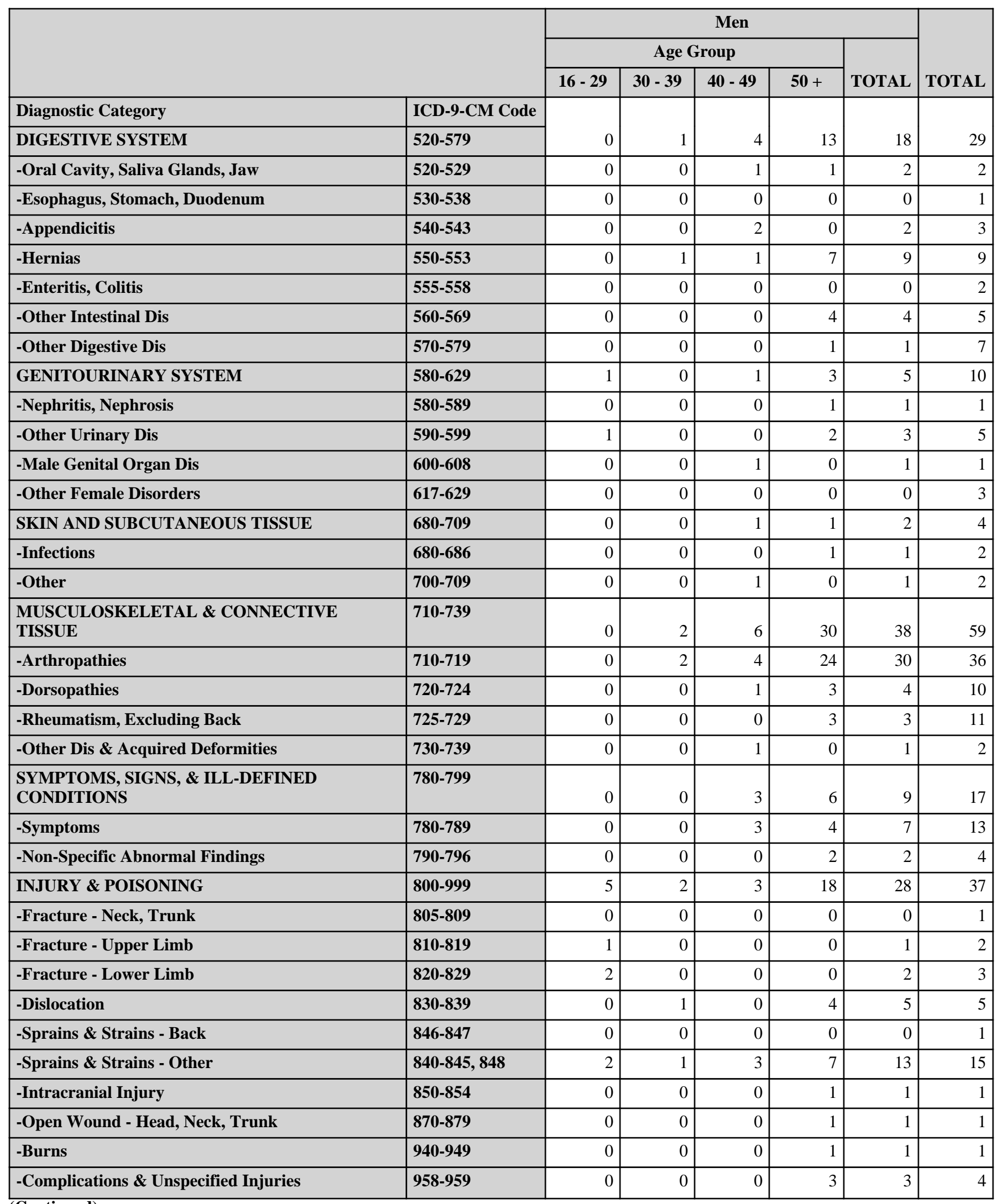

(Continued)

*Only those diagnostic categories and gender/age combinations with at least one occurrence appear in this table. 
Kansas City Plant 2010

Absence Data

Appendix G. Number of Diagnoses in Each Diagnostic Category by Gender and Age*

\begin{tabular}{|c|c|c|c|c|c|c|}
\hline & & \multicolumn{5}{|c|}{ Women } \\
\hline & & \multicolumn{4}{|c|}{ Age Group } & \multirow[b]{2}{*}{ TOTAL } \\
\hline & & $16-29$ & $30-39$ & $40-49$ & $50+$ & \\
\hline Diagnostic Category & ICD-9-CM Code & & & & & \\
\hline -Poisoning - Medicinal/Biological & $960-979$ & 0 & 0 & 0 & 0 & 0 \\
\hline -Complications of Surgical/Medical Care & 996-999 & 0 & 0 & 1 & 1 & 2 \\
\hline $\begin{array}{l}\text { HEALTH STATUS/HEALTH SERVICE } \\
\text { CONTACT }\end{array}$ & V01-V89 & 0 & 0 & 0 & 0 & 0 \\
\hline -Examination \& Investigation & V70-V82 & 0 & 0 & 0 & 0 & 0 \\
\hline
\end{tabular}

*Only those diagnostic categories and gender/age combinations with at least one occurrence appear in this table. 


\section{Kansas City Plant 2010}

Absence Data

Appendix G. Number of Diagnoses in Each Diagnostic Category by Gender and Age*

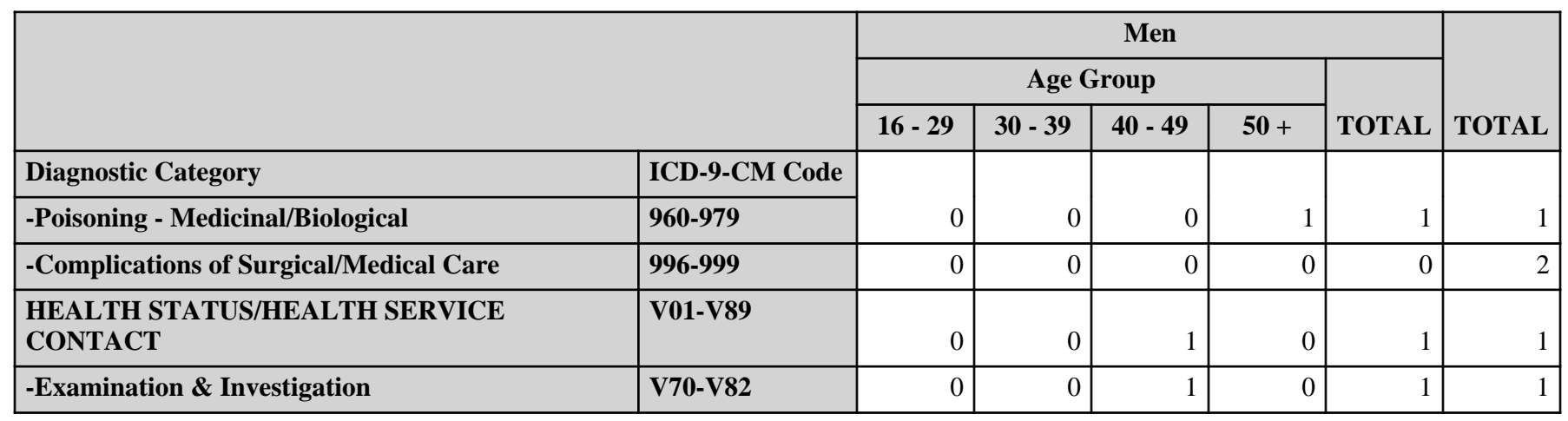

\begin{tabular}{|c|c|c|c|c|c|c|c|c|c|c|c|}
\hline & \multicolumn{5}{|c|}{ Women } & \multicolumn{5}{|c|}{ Men } & \multirow[b]{3}{*}{ TOTAI } \\
\hline & \multicolumn{4}{|c|}{ Age Group } & \multirow[b]{2}{*}{ TOTAL } & \multicolumn{4}{|c|}{ Age Group } & \multirow[b]{2}{*}{ TOTAL } & \\
\hline & $16-29$ & $30-39$ & 40 - 49 & $50+$ & & $16-29$ & $30-39$ & 40 - 49 & $50+$ & & \\
\hline Diagnostic Category & & & & & & & & & & & \\
\hline Total & 5 & 3 & 14 & 64 & 86 & 7 & 6 & 32 & 110 & 155 & 241 \\
\hline
\end{tabular}

*Only those diagnostic categories and gender/age combinations with at least one occurrence appear in this table. 


\section{Kansas City Plant 2010}

Absence Data

Appendix H. Total Number of Calendar Days Absent in Each Diagnostic Category by Gender and Age*

\begin{tabular}{|c|c|c|c|c|c|c|}
\hline & & \multicolumn{5}{|c|}{ Women } \\
\hline & & \multicolumn{4}{|c|}{ Age Group } & \multirow[b]{2}{*}{ TOTAL } \\
\hline & & $16-29$ & $30-39$ & $40-49$ & $\mathbf{5 0 +}$ & \\
\hline Diagnostic Category & ICD-9-CM Code & \multirow[b]{2}{*}{0} & \multirow[b]{2}{*}{19} & \multirow[b]{2}{*}{0} & \multirow[b]{2}{*}{0} & \multirow[b]{2}{*}{19} \\
\hline INFECTIOUS \& PARASITIC DISEASES (DIS) & 001-139 & & & & & \\
\hline MALIGNANT NEOPLASMS & \begin{tabular}{|l|}
$140-208$ \\
$209.0-209.3$ \\
$230-234$
\end{tabular} & 0 & 0 & 0 & 3 & 3 \\
\hline BENIGN \& UNCERTAIN NEOPLASMS & $\begin{array}{l}209.4-209.6 \\
210-229,235-239\end{array}$ & 0 & 0 & 0 & 23 & 23 \\
\hline ENDOCRINE/METABOLIC/IMMUNITY & $240-279$ & 0 & 0 & 0 & 37 & 37 \\
\hline BLOOD \& BLOOD-FORMING ORGANS & $280-289$ & 0 & 0 & 0 & 26 & 26 \\
\hline MENTAL DISORDERS & 290-319 & 9 & 0 & 21 & 0 & 30 \\
\hline NERVOUS SYSTEM (NS) \& SENSE ORGANS & $320-389$ & 0 & 11 & 21 & 103 & 135 \\
\hline CIRCULATORY SYSTEM & $390-459$ & 0 & 0 & 10 & 100 & 110 \\
\hline RESPIRATORY SYSTEM & $460-519$ & 0 & 0 & 0 & 64 & 64 \\
\hline DIGESTIVE SYSTEM & $520-579$ & 0 & 0 & 89 & 107 & 196 \\
\hline GENITOURINARY SYSTEM & $580-629$ & 58 & 0 & 0 & 79 & 137 \\
\hline SKIN AND SUBCUTANEOUS TISSUE & 680-709 & 0 & 19 & 0 & 48 & 67 \\
\hline $\begin{array}{l}\text { MUSCULOSKELETAL \& CONNECTIVE } \\
\text { TISSUE }\end{array}$ & $710-739$ & 0 & 0 & 90 & 449 & 539 \\
\hline $\begin{array}{l}\text { SYMPTOMS, SIGNS, \& ILL-DEFINED } \\
\text { CONDITIONS }\end{array}$ & 780-799 & 13 & 0 & 0 & 55 & 68 \\
\hline INJURY \& POISONING & $800-999$ & 0 & 0 & 81 & 165 & 246 \\
\hline
\end{tabular}

\footnotetext{
*Absences with >1 ICD-9-CM code in the same diagnostic category were counted only once. Only those diagnostic categories and gender/age combinations with at least one occurrence appear in this table.
} 


\section{Kansas City Plant 2010}

Absence Data

Appendix H. Total Number of Calendar Days Absent in Each Diagnostic Category by Gender and Age*

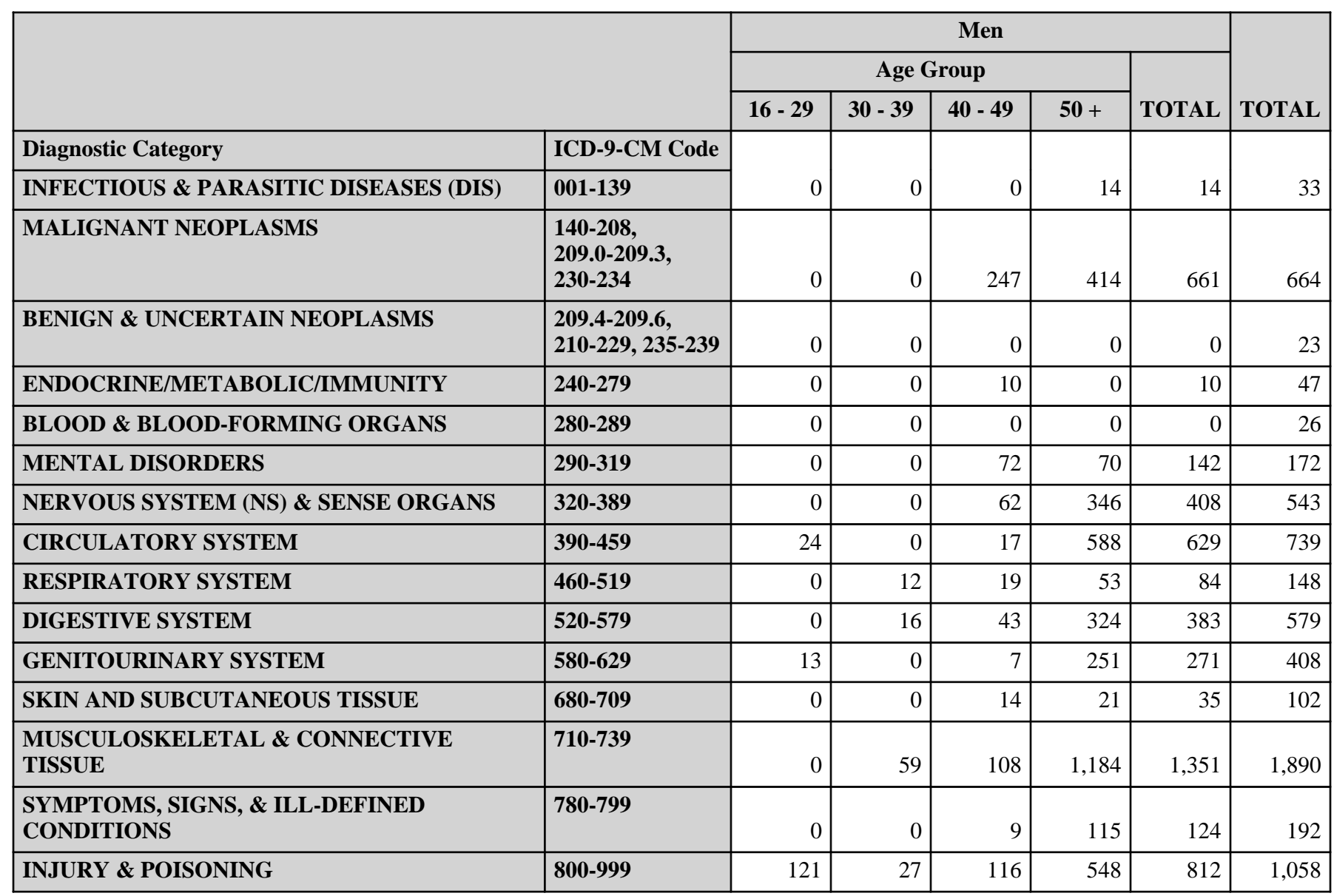

\footnotetext{
*Absences with >1 ICD-9-CM code in the same diagnostic category were counted only once. Only those diagnostic categories and gender/age combinations with at least one occurrence appear in this table.
} 


\section{Kansas City Plant 2010}

Absence Data

Appendix I. Number of Diagnoses in Each Diagnostic Category by Gender and Job Category*

\begin{tabular}{|c|c|c|c|c|c|}
\hline & \multicolumn{4}{|c|}{ Women } \\
\hline & & \multicolumn{4}{|c|}{ Job Category } \\
\hline & & Professional & $\begin{array}{c}\text { Administrative } \\
\text { Support }\end{array}$ & Technical Support & Service \\
\hline Diagnostic Category & ICD-9-CM Code & \multirow[b]{2}{*}{0} & \multirow[b]{2}{*}{1} & \multirow[b]{2}{*}{0} & \multirow[b]{2}{*}{0} \\
\hline INFECTIOUS \& PARASITIC DISEASES (DIS) & 001-139 & & & & \\
\hline MALIGNANT NEOPLASMS & $\begin{array}{l}140-208, \\
209.0-209.3 \\
230-234\end{array}$ & 0 & 0 & 1 & 0 \\
\hline -Breast & $174-175$ & 0 & 0 & 1 & 0 \\
\hline BENIGN \& UNCERTAIN NEOPLASMS & $\begin{array}{l}209.4-209.6 \\
210-229,235-239\end{array}$ & 0 & 0 & 1 & 0 \\
\hline -Other Endocrine Gland Dis & $249-259$ & 0 & 0 & 1 & 0 \\
\hline -Other Metabolic \& Immunity Disorders & 270-279 & 0 & 0 & 1 & 0 \\
\hline BLOOD \& BLOOD-FORMING ORGANS & $280-289$ & 0 & 0 & 1 & 0 \\
\hline MENTAL DISORDERS & $290-319$ & 0 & 0 & 4 & 0 \\
\hline -Psychoses & 290-299 & 0 & 0 & 0 & 0 \\
\hline -Non-Psychotic Disorders & $300-302,306-316$ & 0 & 0 & 4 & 0 \\
\hline NERVOUS SYSTEM (NS) \& SENSE ORGANS & $320-389$ & 2 & 2 & 0 & 1 \\
\hline -Inflammatory Dis of Central NS & $320-326$ & 0 & 1 & 0 & 0 \\
\hline -Dis of Veins, Lymphatics, Other & $451-459$ & 1 & 0 & 0 & 0 \\
\hline RESPIRATORY SYSTEM & $460-519$ & 0 & 2 & 0 & 1 \\
\hline -Other Dis Upper Respiratory Tract & $470-478$ & 0 & 1 & 0 & 0 \\
\hline -Pneumonia \& Influenza & $480-488$ & 0 & 1 & 0 & 0 \\
\hline -Chronic Obstructive Dis & $490-496$ & 0 & 0 & 0 & 0 \\
\hline -Other Respiratory Dis & $510-519$ & 0 & 0 & 0 & 1 \\
\hline DIGESTIVE SYSTEM & $520-579$ & 1 & 4 & 0 & 2 \\
\hline -Esophagus, Stomach, Duodenum & \begin{tabular}{|c|}
$530-538$ \\
\end{tabular} & 0 & 0 & 0 & 0 \\
\hline -Appendicitis & $540-543$ & 0 & 1 & 0 & 0 \\
\hline -Enteritis, Colitis & $555-558$ & 0 & 0 & 0 & 0 \\
\hline -Other Intestinal Dis & $560-569$ & 0 & 1 & 0 & 0 \\
\hline -Other Digestive Dis & \begin{tabular}{|l|}
$570-579$ \\
\end{tabular} & 1 & 2 & 0 & 2 \\
\hline GENITOURINARY SYSTEM & $580-629$ & 3 & 0 & 1 & 1 \\
\hline
\end{tabular}

(Continued)

*Only those diagnostic categories and gender/job category combinations with at least one occurrence appear in this table. 


\section{Kansas City Plant 2010}

Absence Data

Appendix I. Number of Diagnoses in Each Diagnostic Category by Gender and Job Category*

\begin{tabular}{|c|c|c|c|c|c|}
\hline & & \multicolumn{4}{|c|}{ Women } \\
\hline & & \multicolumn{3}{|c|}{ Job Category } & \multirow[b]{2}{*}{ TOTAL } \\
\hline & & Security and Fire & Crafts & Line Operators & \\
\hline Diagnostic Category & ICD-9-CM Code & \multirow[b]{2}{*}{0} & \multirow[b]{2}{*}{0} & \multirow[b]{2}{*}{0} & \multirow[b]{2}{*}{1} \\
\hline INFECTIOUS \& PARASITIC DISEASES (DIS) & 001-139 & & & & \\
\hline -Other Bacterial Dis & $030-041$ & 0 & 0 & 0 & 1 \\
\hline MALIGNANT NEOPLASMS & $\begin{array}{l}140-208 \\
209.0-209.3 \\
230-234\end{array}$ & 0 & 0 & 0 & 1 \\
\hline -Breast & 174-175 & 0 & 0 & 0 & 1 \\
\hline BENIGN \& UNCERTAIN NEOPLASMS & \begin{tabular}{|l|}
$209.4-209.6$ \\
$210-229,235-239$
\end{tabular} & 0 & 0 & 1 & 2 \\
\hline ENDOCRINE/METABOLIC/IMMUNITY & $240-279$ & 0 & 0 & 1 & 4 \\
\hline -Thyroid Gland Disorders & $240-246$ & 0 & 0 & 1 & 2 \\
\hline -Other Endocrine Gland Dis & $249-259$ & 0 & 0 & 0 & 1 \\
\hline -Other Metabolic \& Immunity Disorders & $270-279$ & 0 & 0 & 0 & 1 \\
\hline BLOOD \& BLOOD-FORMING ORGANS & $280-289$ & 0 & 0 & 0 & 1 \\
\hline MENTAL DISORDERS & $290-319$ & 0 & 0 & 2 & 6 \\
\hline -Psychoses & $290-299$ & 0 & 0 & 1 & 1 \\
\hline -Non-Psychotic Disorders & $300-302,306-316$ & 0 & 0 & 1 & 5 \\
\hline NERVOUS SYSTEM (NS) \& SENSE ORGANS & $320-389$ & 1 & 0 & 0 & 6 \\
\hline -Inflammatory Dis of Central NS & $320-326$ & 0 & 0 & 0 & 1 \\
\hline -Other Disorders of Central NS & $340-349$ & 0 & 0 & 0 & 1 \\
\hline -Disorders of Peripheral NS & $350-359$ & 1 & 0 & 0 & 2 \\
\hline -Disorders of Eye & $360-379$ & 0 & 0 & 0 & 1 \\
\hline -Diseases of Ear \& Mastoid & 380-389 & 0 & 0 & 0 & 1 \\
\hline CIRCULATORY SYSTEM & $390-459$ & 0 & 0 & 0 & 4 \\
\hline -Ischemic Heart Dis & $410-414$ & 0 & 0 & 0 & 3 \\
\hline -Dis of Veins, Lymphatics, Other & 451-459 & 0 & 0 & 0 & 1 \\
\hline RESPIRATORY SYSTEM & $460-519$ & 0 & 2 & 0 & 5 \\
\hline -Other Dis Upper Respiratory Tract & $470-478$ & 0 & 1 & 0 & 2 \\
\hline -Pneumonia \& Influenza & $480-488$ & 0 & 0 & 0 & 1 \\
\hline -Chronic Obstructive Dis & $490-496$ & 0 & 1 & 0 & 1 \\
\hline -Other Respiratory Dis & $510-519$ & 0 & 0 & 0 & 1 \\
\hline DIGESTIVE SYSTEM & $520-579$ & 0 & 1 & 3 & 11 \\
\hline -Esophagus, Stomach, Duodenum & $530-538$ & 0 & 0 & 1 & 1 \\
\hline -Appendicitis & $540-543$ & 0 & 0 & 0 & 1 \\
\hline -Enteritis, Colitis & $555-558$ & 0 & 0 & 2 & 2 \\
\hline -Other Intestinal Dis & $560-569$ & 0 & 0 & 0 & 1 \\
\hline -Other Digestive Dis & $570-579$ & 0 & 1 & 0 & 6 \\
\hline GENITOURINARY SYSTEM & $580-629$ & 0 & 0 & 0 & 5 \\
\hline
\end{tabular}

(Continued)

*Only those diagnostic categories and gender/job category combinations with at least one occurrence appear in this table. 


\section{Kansas City Plant 2010}

Absence Data

Appendix I. Number of Diagnoses in Each Diagnostic Category by Gender and Job Category*

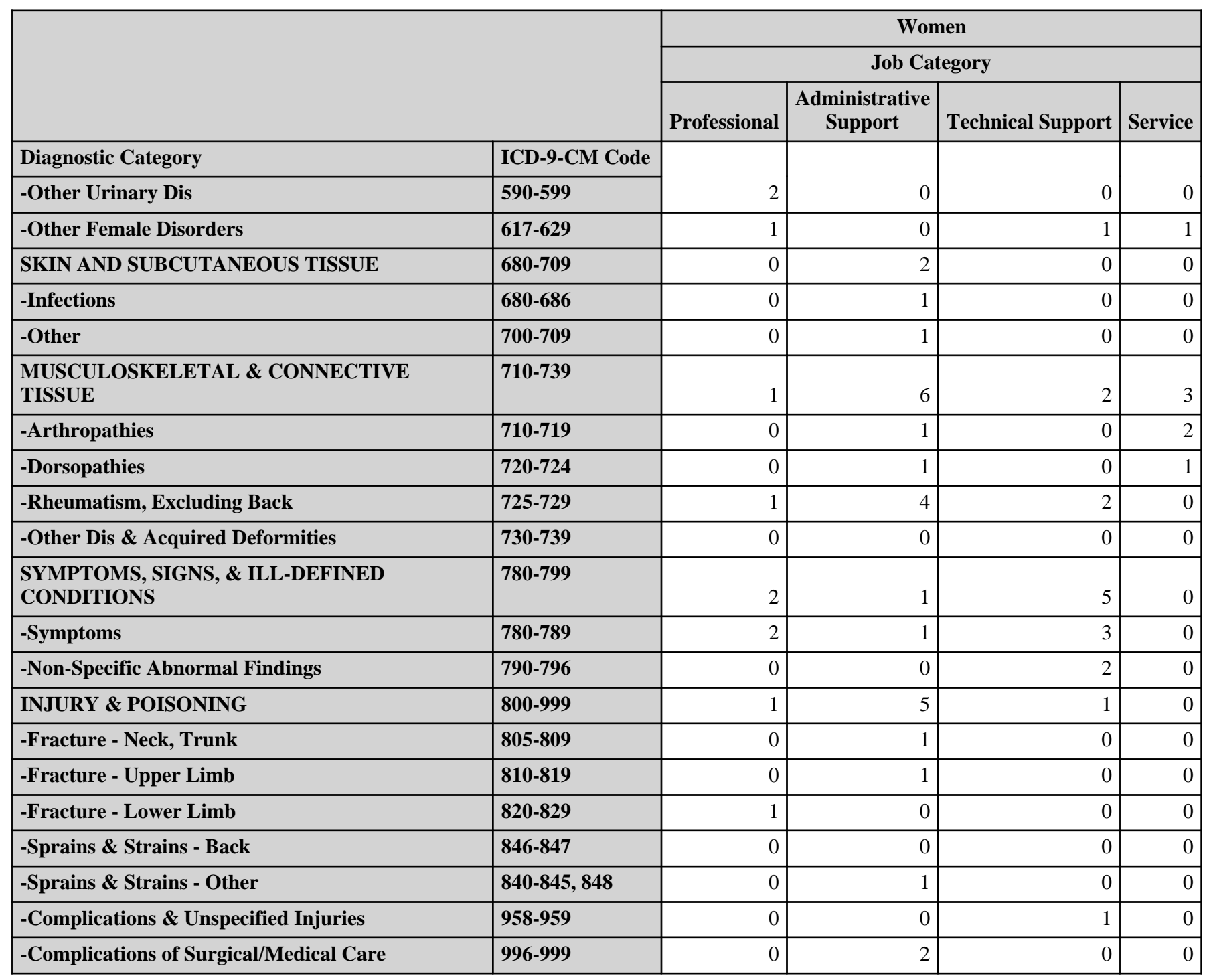

*Only those diagnostic categories and gender/job category combinations with at least one occurrence appear in this table. 


\section{Kansas City Plant 2010}

Absence Data

Appendix I. Number of Diagnoses in Each Diagnostic Category by Gender and Job Category*

\begin{tabular}{|c|c|c|c|c|c|}
\hline & & \multicolumn{4}{|c|}{ Women } \\
\hline & & \multicolumn{3}{|c|}{ Job Category } & \multirow[b]{2}{*}{ TOTAL } \\
\hline & & Security and Fire & Crafts & Line Operators & \\
\hline Diagnostic Category & ICD-9-CM Code & \multirow[b]{2}{*}{0} & \multirow[b]{2}{*}{0} & \multirow[b]{2}{*}{0} & \multirow[b]{2}{*}{2} \\
\hline -Other Urinary Dis & $590-599$ & & & & \\
\hline -Other Female Disorders & $617-629$ & 0 & 0 & 0 & 3 \\
\hline SKIN AND SUBCUTANEOUS TISSUE & 680-709 & 0 & 0 & 0 & 2 \\
\hline -Infections & $680-686$ & 0 & 0 & 0 & 1 \\
\hline -Other & $700-709$ & 0 & 0 & 0 & 1 \\
\hline $\begin{array}{l}\text { MUSCULOSKELETAL \& CONNECTIVE } \\
\text { TISSUE }\end{array}$ & $710-739$ & 0 & 3 & 6 & 21 \\
\hline -Arthropathies & $710-719$ & 0 & 1 & 2 & 6 \\
\hline -Dorsopathies & $720-724$ & 0 & 1 & 3 & 6 \\
\hline -Rheumatism, Excluding Back & 725-729 & 0 & 0 & 1 & 8 \\
\hline -Other Dis \& Acquired Deformities & $730-739$ & 0 & 1 & 0 & 1 \\
\hline $\begin{array}{l}\text { SYMPTOMS, SIGNS, \& ILL-DEFINED } \\
\text { CONDITIONS }\end{array}$ & 780-799 & 0 & 0 & 0 & 8 \\
\hline -Symptoms & 780-789 & 0 & 0 & 0 & 6 \\
\hline -Non-Specific Abnormal Findings & $790-796$ & 0 & 0 & 0 & 2 \\
\hline INJURY \& POISONING & $800-999$ & 1 & 0 & 1 & 9 \\
\hline -Fracture - Neck, Trunk & $805-809$ & 0 & 0 & 0 & 1 \\
\hline -Fracture - Upper Limb & $810-819$ & 0 & 0 & 0 & 1 \\
\hline -Fracture - Lower Limb & $820-829$ & 0 & 0 & 0 & 1 \\
\hline -Sprains \& Strains - Back & 846-847 & 0 & 0 & 1 & 1 \\
\hline -Sprains \& Strains - Other & $840-845,848$ & 1 & 0 & 0 & 2 \\
\hline -Complications \& Unspecified Injuries & 958-959 & 0 & 0 & 0 & 1 \\
\hline -Complications of Surgical/Medical Care & 996-999 & 0 & 0 & 0 & 2 \\
\hline
\end{tabular}

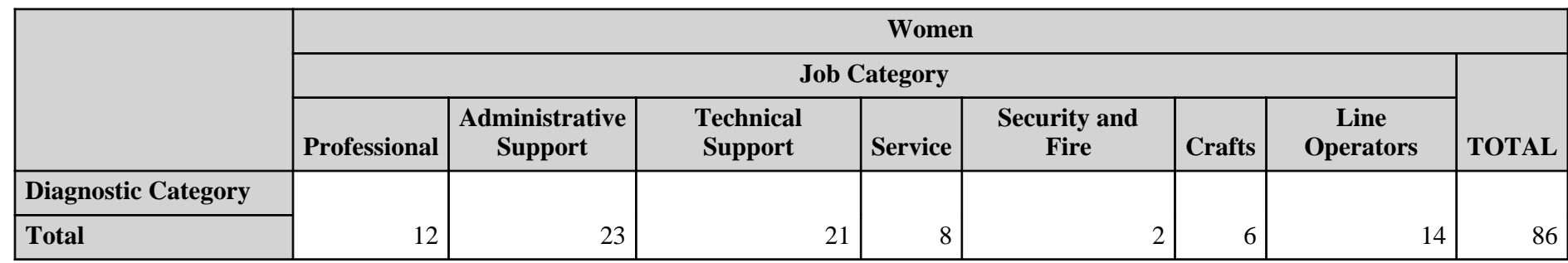

*Only those diagnostic categories and gender/job category combinations with at least one occurrence appear in this table. 


\section{Kansas City Plant 2010}

Absence Data

Appendix I. Number of Diagnoses in Each Diagnostic Category by Gender and Job Category*

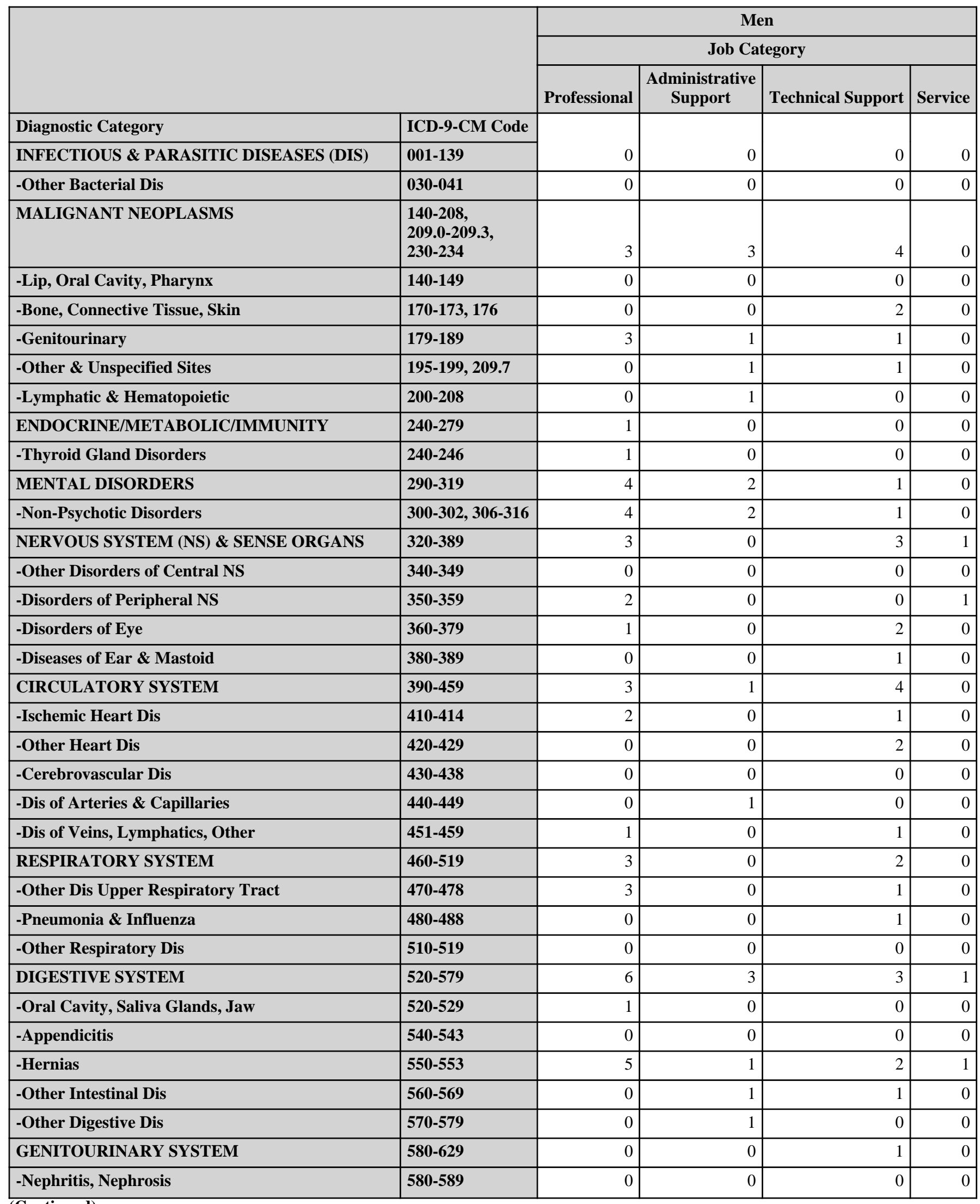

(Continued)

*Only those diagnostic categories and gender/job category combinations with at least one occurrence appear in this table. 


\section{Kansas City Plant 2010}

Absence Data

Appendix I. Number of Diagnoses in Each Diagnostic Category by Gender and Job Category*

\begin{tabular}{|c|c|c|c|c|c|}
\hline & & \multicolumn{4}{|c|}{ Men } \\
\hline & & \multicolumn{3}{|c|}{ Job Category } & \multirow[b]{2}{*}{ TOTAL } \\
\hline & & Security and Fire & Crafts & Line Operators & \\
\hline Diagnostic Category & ICD-9-CM Code & \multirow[b]{2}{*}{0} & \multirow[b]{2}{*}{1} & \multirow[b]{2}{*}{0} & \multirow[b]{2}{*}{1} \\
\hline INFECTIOUS \& PARASITIC DISEASES (DIS) & 001-139 & & & & \\
\hline -Other Bacterial Dis & $030-041$ & 0 & 1 & 0 & 1 \\
\hline MALIGNANT NEOPLASMS & $\begin{array}{l}140-208 \\
209.0-209.3 \\
230-234\end{array}$ & 0 & 0 & 2 & 12 \\
\hline -Lip, Oral Cavity, Pharynx & $140-149$ & 0 & 0 & 1 & 1 \\
\hline -Bone, Connective Tissue, Skin & $170-173,176$ & 0 & 0 & 0 & 2 \\
\hline -Genitourinary & 179-189 & 0 & 0 & 0 & 5 \\
\hline -Other \& Unspecified Sites & 195-199, 209.7 & 0 & 0 & 1 & 3 \\
\hline -Lymphatic \& Hematopoietic & $200-208$ & 0 & 0 & 0 & 1 \\
\hline ENDOCRINE/METABOLIC/IMMUNITY & $240-279$ & 0 & 0 & 0 & 1 \\
\hline -Thyroid Gland Disorders & $240-246$ & 0 & 0 & 0 & 1 \\
\hline MENTAL DISORDERS & $290-319$ & 0 & 0 & 0 & 7 \\
\hline -Non-Psychotic Disorders & $300-302,306-316$ & 0 & 0 & 0 & 7 \\
\hline NERVOUS SYSTEM (NS) \& SENSE ORGANS & 320-389 & 0 & 2 & 0 & 9 \\
\hline -Other Disorders of Central NS & 340-349 & 0 & 1 & 0 & 1 \\
\hline -Disorders of Peripheral NS & $350-359$ & 0 & 0 & 0 & 3 \\
\hline -Disorders of Eye & $360-379$ & 0 & 1 & 0 & 4 \\
\hline -Diseases of Ear \& Mastoid & $380-389$ & 0 & 0 & 0 & 1 \\
\hline CIRCULATORY SYSTEM & $390-459$ & 1 & 7 & 1 & 17 \\
\hline -Ischemic Heart Dis & $410-414$ & 1 & 5 & 1 & 10 \\
\hline -Other Heart Dis & $420-429$ & 0 & 1 & 0 & 3 \\
\hline -Cerebrovascular Dis & $430-438$ & 0 & 1 & 0 & 1 \\
\hline -Dis of Arteries \& Capillaries & $440-449$ & 0 & 0 & 0 & 1 \\
\hline -Dis of Veins, Lymphatics, Other & 451-459 & 0 & 0 & 0 & 2 \\
\hline RESPIRATORY SYSTEM & $460-519$ & 0 & 1 & 1 & 7 \\
\hline -Other Dis Upper Respiratory Tract & $470-478$ & 0 & 0 & 1 & 5 \\
\hline -Pneumonia \& Influenza & $480-488$ & 0 & 0 & 0 & 1 \\
\hline -Other Respiratory Dis & $510-519$ & 0 & 1 & 0 & 1 \\
\hline DIGESTIVE SYSTEM & $520-579$ & 0 & 4 & 1 & 18 \\
\hline -Oral Cavity, Saliva Glands, Jaw & $520-529$ & 0 & 0 & 1 & 2 \\
\hline -Appendicitis & $540-543$ & 0 & 2 & 0 & 2 \\
\hline -Hernias & $550-553$ & 0 & 0 & 0 & 9 \\
\hline -Other Intestinal Dis & $560-569$ & 0 & 2 & 0 & 4 \\
\hline -Other Digestive Dis & $570-579$ & 0 & 0 & 0 & 1 \\
\hline GENITOURINARY SYSTEM & $580-629$ & 0 & 3 & 1 & 5 \\
\hline -Nephritis, Nephrosis & $580-589$ & 0 & 1 & 0 & 1 \\
\hline
\end{tabular}

(Continued)

*Only those diagnostic categories and gender/job category combinations with at least one occurrence appear in this table. 


\section{Kansas City Plant 2010}

Absence Data

Appendix I. Number of Diagnoses in Each Diagnostic Category by Gender and Job Category*

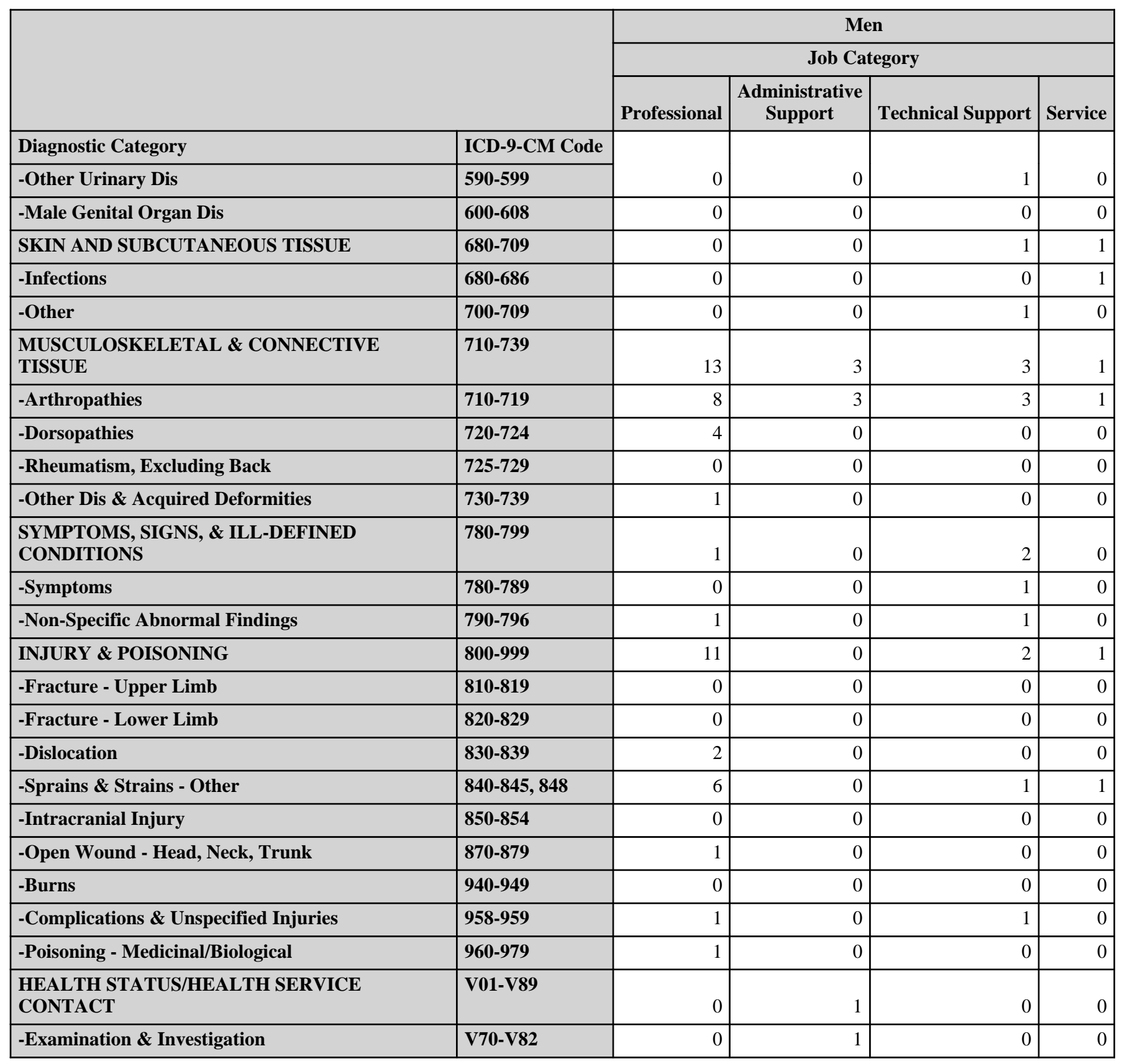

*Only those diagnostic categories and gender/job category combinations with at least one occurrence appear in this table. 


\section{Kansas City Plant 2010}

Absence Data

Appendix I. Number of Diagnoses in Each Diagnostic Category by Gender and Job Category*

\begin{tabular}{|c|c|c|c|c|c|}
\hline & & \multicolumn{4}{|c|}{ Men } \\
\hline & & \multicolumn{3}{|c|}{ Job Category } & \multirow[b]{2}{*}{ TOTAL } \\
\hline & & Security and Fire & Crafts & Line Operators & \\
\hline Diagnostic Category & ICD-9-CM Code & \multirow[b]{2}{*}{0} & \multirow[b]{2}{*}{2} & \multirow[b]{2}{*}{0} & \multirow[b]{2}{*}{3} \\
\hline -Other Urinary Dis & $590-599$ & & & & \\
\hline -Male Genital Organ Dis & $600-608$ & 0 & 0 & 1 & 1 \\
\hline SKIN AND SUBCUTANEOUS TISSUE & $680-709$ & 0 & 0 & 0 & 2 \\
\hline -Infections & $680-686$ & 0 & 0 & 0 & 1 \\
\hline -Other & 700-709 & 0 & 0 & 0 & 1 \\
\hline $\begin{array}{l}\text { MUSCULOSKELETAL \& CONNECTIVE } \\
\text { TISSUE }\end{array}$ & $710-739$ & 0 & 15 & 3 & 38 \\
\hline -Arthropathies & $710-719$ & 0 & 13 & 2 & 30 \\
\hline -Dorsopathies & $720-724$ & 0 & 0 & 0 & 4 \\
\hline -Rheumatism, Excluding Back & 725-729 & 0 & 2 & 1 & 3 \\
\hline -Other Dis \& Acquired Deformities & $730-739$ & 0 & 0 & 0 & 1 \\
\hline $\begin{array}{l}\text { SYMPTOMS, SIGNS, \& ILL-DEFINED } \\
\text { CONDITIONS }\end{array}$ & $780-799$ & 0 & 5 & 1 & 9 \\
\hline -Symptoms & 780-789 & 0 & 5 & 1 & 7 \\
\hline -Non-Specific Abnormal Findings & $790-796$ & 0 & 0 & 0 & 2 \\
\hline INJURY \& POISONING & $800-999$ & 1 & 8 & 5 & 28 \\
\hline -Fracture - Upper Limb & $810-819$ & 0 & 0 & 1 & 1 \\
\hline -Fracture - Lower Limb & $820-829$ & 0 & 0 & 2 & 2 \\
\hline -Dislocation & $830-839$ & 0 & 3 & 0 & 5 \\
\hline -Sprains \& Strains - Other & $840-845,848$ & 1 & 4 & 0 & 13 \\
\hline -Intracranial Injury & $850-854$ & 0 & 0 & 1 & 1 \\
\hline -Open Wound - Head, Neck, Trunk & $870-879$ & 0 & 0 & 0 & 1 \\
\hline -Burns & $940-949$ & 0 & 1 & 0 & 1 \\
\hline -Complications \& Unspecified Injuries & 958-959 & 0 & 0 & 1 & 3 \\
\hline -Poisoning - Medicinal/Biological & $960-979$ & 0 & 0 & 0 & 1 \\
\hline $\begin{array}{l}\text { HEALTH STATUS/HEALTH SERVICE } \\
\text { CONTACT }\end{array}$ & V01-V89 & 0 & 0 & 0 & 1 \\
\hline -Examination \& Investigation & V70-V82 & 0 & 0 & 0 & 1 \\
\hline
\end{tabular}

\begin{tabular}{|c|c|c|c|c|c|c|c|c|}
\hline & \multicolumn{8}{|c|}{ Men } \\
\hline & \multicolumn{7}{|c|}{ Job Category } & \multirow[b]{2}{*}{ TOTAL } \\
\hline & Professional & $\begin{array}{c}\text { Administrative } \\
\text { Support }\end{array}$ & $\begin{array}{c}\text { Technical } \\
\text { Support }\end{array}$ & Service & $\begin{array}{l}\text { Security and } \\
\text { Fire }\end{array}$ & Crafts & $\begin{array}{c}\text { Line } \\
\text { Operators }\end{array}$ & \\
\hline \begin{tabular}{|l|} 
Diagnostic Category \\
\end{tabular} & \multirow[b]{2}{*}{48} & \multirow[b]{2}{*}{13} & \multirow[b]{2}{*}{26} & \multirow[b]{2}{*}{5} & \multirow[b]{2}{*}{2} & \multirow[b]{2}{*}{46} & \multirow[b]{2}{*}{15} & \multirow[b]{2}{*}{155} \\
\hline Total & & & & & & & & \\
\hline
\end{tabular}

*Only those diagnostic categories and gender/job category combinations with at least one occurrence appear in this table. 


\section{Kansas City Plant 2010}

Absence Data

Appendix J. Total Number of Calendar Days Absent in Each Diagnostic Category by Gender and Job Category*

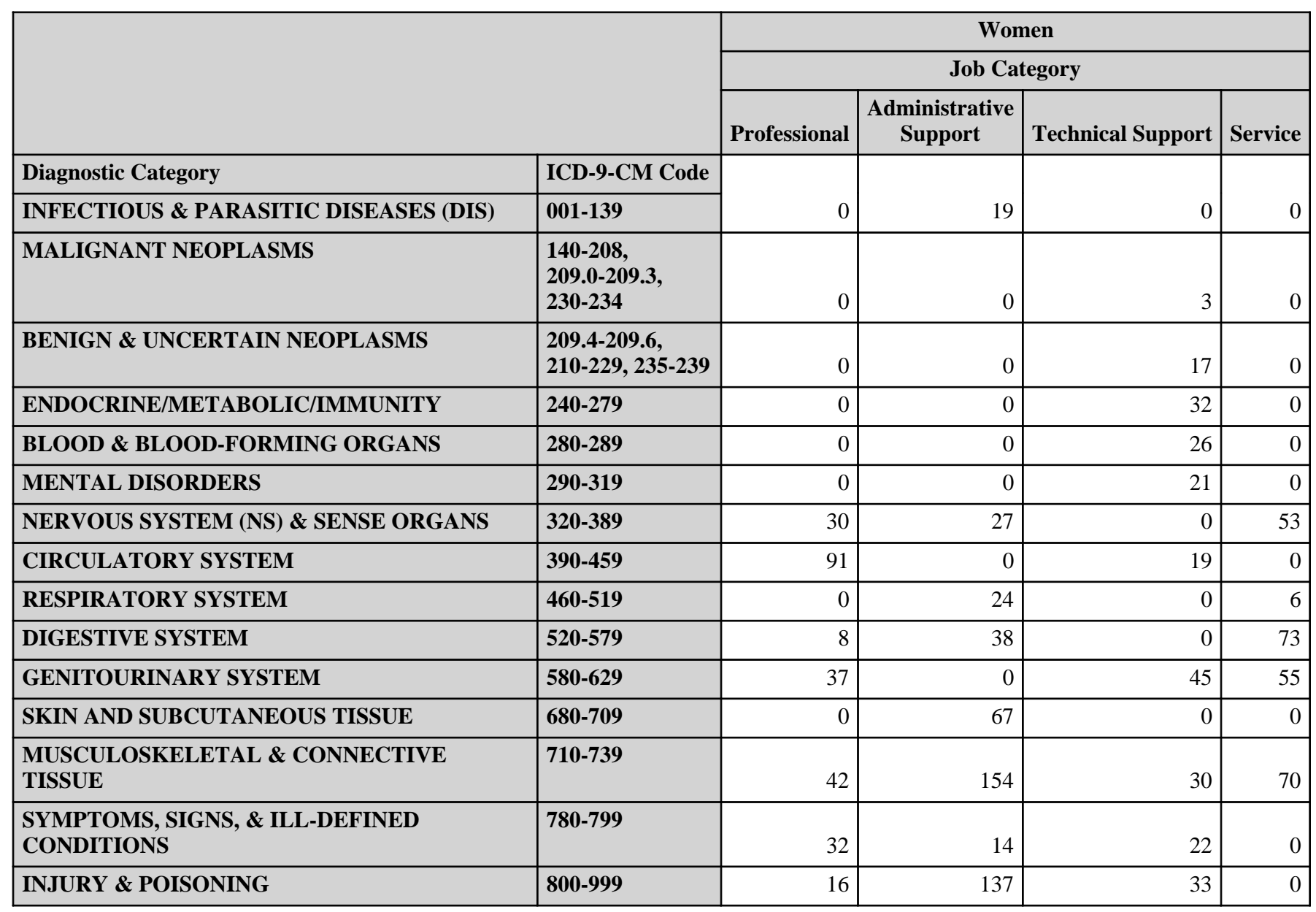

\footnotetext{
*Absences with >1 ICD-9-CM code in the same diagnostic category were counted only once. Only those diagnostic categories and gender/job category combinations with at least one occurrence appear in this table.
} 


\section{Kansas City Plant 2010}

Absence Data

Appendix J. Total Number of Calendar Days Absent in Each Diagnostic Category by Gender and Job Category*

\begin{tabular}{|c|c|c|c|c|c|}
\hline & & \multicolumn{4}{|c|}{ Women } \\
\hline & & \multicolumn{3}{|c|}{ Job Category } & \multirow[b]{2}{*}{ TOTAL } \\
\hline & & Security and Fire & Crafts & Line Operators & \\
\hline Diagnostic Category & ICD-9-CM Code & \multirow[b]{2}{*}{0} & \multirow[b]{2}{*}{0} & \multirow[b]{2}{*}{0} & \multirow[b]{2}{*}{19} \\
\hline INFECTIOUS \& PARASITIC DISEASES (DIS) & 001-139 & & & & \\
\hline MALIGNANT NEOPLASMS & $\begin{array}{l}140-208 \\
209.0-209.3 \\
230-234\end{array}$ & 0 & 0 & 0 & 3 \\
\hline BENIGN \& UNCERTAIN NEOPLASMS & $\begin{array}{l}\text { 209.4-209.6, } \\
210-229,235-239\end{array}$ & 0 & 0 & 6 & 23 \\
\hline ENDOCRINE/METABOLIC/IMMUNITY & 240-279 & 0 & 0 & 5 & 37 \\
\hline BLOOD \& BLOOD-FORMING ORGANS & 280-289 & 0 & 0 & 0 & 26 \\
\hline MENTAL DISORDERS & 290-319 & 0 & 0 & 9 & 30 \\
\hline NERVOUS SYSTEM (NS) \& SENSE ORGANS & 320-389 & 25 & 0 & 0 & 135 \\
\hline CIRCULATORY SYSTEM & $390-459$ & 0 & 0 & 0 & 110 \\
\hline RESPIRATORY SYSTEM & $460-519$ & 0 & 34 & 0 & 64 \\
\hline DIGESTIVE SYSTEM & $520-579$ & 0 & 32 & 45 & 196 \\
\hline GENITOURINARY SYSTEM & $580-629$ & 0 & 0 & 0 & 137 \\
\hline SKIN AND SUBCUTANEOUS TISSUE & 680-709 & 0 & 0 & 0 & 67 \\
\hline $\begin{array}{l}\text { MUSCULOSKELETAL \& CONNECTIVE } \\
\text { TISSUE }\end{array}$ & 710-739 & 0 & 82 & 161 & 539 \\
\hline $\begin{array}{l}\text { SYMPTOMS, SIGNS, \& ILL-DEFINED } \\
\text { CONDITIONS }\end{array}$ & $780-799$ & 0 & 0 & 0 & 68 \\
\hline INJURY \& POISONING & 800-999 & 11 & 0 & 49 & 246 \\
\hline
\end{tabular}

\footnotetext{
*Absences with >1 ICD-9-CM code in the same diagnostic category were counted only once. Only those diagnostic categories and gender/job category combinations with at least one occurrence appear in this table.
} 


\section{Kansas City Plant 2010}

Absence Data

Appendix J. Total Number of Calendar Days Absent in Each Diagnostic Category by Gender and Job Category*

\begin{tabular}{|c|c|c|c|c|c|}
\hline & \multicolumn{4}{|c|}{ Men } \\
\hline & & \multicolumn{4}{|c|}{ Job Category } \\
\hline & & Professional & $\begin{array}{c}\text { Administrative } \\
\text { Support }\end{array}$ & Technical Support & Service \\
\hline Diagnostic Category & ICD-9-CM Code & \multirow[b]{2}{*}{0} & \multirow[b]{2}{*}{0} & \multirow[b]{2}{*}{0} & \multirow[b]{2}{*}{0} \\
\hline INFECTIOUS \& PARASITIC DISEASES (DIS) & 001-139 & & & & \\
\hline MALIGNANT NEOPLASMS & \begin{tabular}{|l|}
$140-208$, \\
$209.0-209.3$ \\
$230-234$
\end{tabular} & 118 & 146 & 200 & 0 \\
\hline ENDOCRINE/METABOLIC/IMMUNITY & 240-279 & 10 & 0 & 0 & 0 \\
\hline MENTAL DISORDERS & 290-319 & 60 & 72 & 10 & 0 \\
\hline NERVOUS SYSTEM (NS) \& SENSE ORGANS & 320-389 & 206 & 0 & 73 & 97 \\
\hline CIRCULATORY SYSTEM & 390-459 & 36 & 28 & 78 & 0 \\
\hline RESPIRATORY SYSTEM & $460-519$ & 25 & 0 & 16 & 0 \\
\hline DIGESTIVE SYSTEM & $520-579$ & 64 & 46 & 92 & 97 \\
\hline GENITOURINARY SYSTEM & 580-629 & 0 & 0 & 2 & 0 \\
\hline SKIN AND SUBCUTANEOUS TISSUE & 680-709 & 0 & 0 & 14 & 21 \\
\hline $\begin{array}{l}\text { MUSCULOSKELETAL \& CONNECTIVE } \\
\text { TISSUE }\end{array}$ & 710-739 & 377 & 82 & 118 & 8 \\
\hline $\begin{array}{l}\text { SYMPTOMS, SIGNS, \& ILL-DEFINED } \\
\text { CONDITIONS }\end{array}$ & 780-799 & 28 & 0 & 43 & 0 \\
\hline INJURY \& POISONING & $800-999$ & 191 & 0 & 84 & 97 \\
\hline
\end{tabular}

\footnotetext{
*Absences with >1 ICD-9-CM code in the same diagnostic category were counted only once. Only those diagnostic categories and gender/job category combinations with at least one occurrence appear in this table.
} 


\section{Kansas City Plant 2010}

Absence Data

Appendix J. Total Number of Calendar Days Absent in Each Diagnostic Category by Gender and Job Category*

\begin{tabular}{|c|c|c|c|c|c|}
\hline & & \multicolumn{4}{|c|}{ Men } \\
\hline & & \multicolumn{3}{|c|}{ Job Category } & \multirow[b]{2}{*}{ TOTAL } \\
\hline & & Security and Fire & Crafts & Line Operators & \\
\hline Diagnostic Category & ICD-9-CM Code & \multirow[b]{2}{*}{0} & \multirow[b]{2}{*}{14} & \multirow[b]{2}{*}{0} & \multirow[b]{2}{*}{14} \\
\hline INFECTIOUS \& PARASITIC DISEASES (DIS) & 001-139 & & & & \\
\hline MALIGNANT NEOPLASMS & $\begin{array}{l}140-208, \\
209.0-209.3 \\
230-234\end{array}$ & 0 & 0 & 197 & 661 \\
\hline ENDOCRINE/METABOLIC/IMMUNITY & 240-279 & 0 & 0 & 0 & 10 \\
\hline MENTAL DISORDERS & $290-319$ & 0 & 0 & 0 & 142 \\
\hline NERVOUS SYSTEM (NS) \& SENSE ORGANS & $320-389$ & 0 & 32 & 0 & 408 \\
\hline CIRCULATORY SYSTEM & $390-459$ & 24 & 363 & 100 & 629 \\
\hline RESPIRATORY SYSTEM & $460-519$ & 0 & 38 & 5 & 84 \\
\hline DIGESTIVE SYSTEM & $520-579$ & 0 & 81 & 3 & 383 \\
\hline GENITOURINARY SYSTEM & $580-629$ & 0 & 262 & 7 & 271 \\
\hline SKIN AND SUBCUTANEOUS TISSUE & $680-709$ & 0 & 0 & 0 & 35 \\
\hline $\begin{array}{l}\text { MUSCULOSKELETAL \& CONNECTIVE } \\
\text { TISSUE }\end{array}$ & 710-739 & 0 & 623 & 143 & 1,351 \\
\hline $\begin{array}{l}\text { SYMPTOMS, SIGNS, \& ILL-DEFINED } \\
\text { CONDITIONS }\end{array}$ & 780-799 & 0 & 41 & 12 & 124 \\
\hline INJURY \& POISONING & 800-999 & 91 & 216 & 133 & 812 \\
\hline
\end{tabular}

\footnotetext{
*Absences with >1 ICD-9-CM code in the same diagnostic category were counted only once. Only those diagnostic categories and gender/job category combinations with at least one occurrence appear in this table.
} 


\section{Kansas City Plant 2010}

Absence Data

Appendix K. Age-Adjusted Illness and Injury Rates by Diagnostic Category*

Part 1. Men

\begin{tabular}{|c|c|c|c|c|c|}
\hline & & $\begin{array}{l}\text { Number of } \\
\text { Diagnoses }\end{array}$ & $\begin{array}{c}\text { Age-Adjusted } \\
\text { Rate per } 1,000 * *\end{array}$ & $\begin{array}{c}\text { Lower } 95 \% \\
\text { Confidence } \\
\text { Limit per 1,000 }\end{array}$ & $\begin{array}{c}\text { Upper } 95 \% \\
\text { Confidence } \\
\text { Limit per } 1,000\end{array}$ \\
\hline Diagnostic Category & ICD-9-CM Code & & & & \\
\hline INFECTIOUS \& PARASITIC DISEASES (DIS) & 001-139 & 1 & 0.2 & 0.0 & 1.4 \\
\hline -Other Bacterial Dis & $030-041$ & 1 & 0.2 & 0.0 & 1.4 \\
\hline MALIGNANT NEOPLASMS & $\begin{array}{l}140-208 \\
209.0-209.3 \\
230-234\end{array}$ & 12 & 4.8 & 2.6 & 8.9 \\
\hline -Lip, Oral Cavity, Pharynx & 140-149 & 1 & 0.6 & 0.1 & 4.3 \\
\hline -Bone, Connective Tissue, Skin & $170-173,176$ & 2 & 1.2 & 0.3 & 4.8 \\
\hline -Genitourinary & 179-189 & 5 & 1.6 & 0.6 & 4.0 \\
\hline -Other \& Unspecified Sites & $195-199,209.7$ & 3 & 1.2 & 0.4 & 4.1 \\
\hline -Lymphatic \& Hematopoietic & $200-208$ & 1 & 0.2 & 0.0 & 1.4 \\
\hline ENDOCRINE/METABOLIC/IMMUNITY & $240-279$ & 1 & 0.6 & 0.1 & 4.3 \\
\hline -Thyroid Gland Disorders & $240-246$ & 1 & 0.6 & 0.1 & 4.3 \\
\hline MENTAL DISORDERS & $290-319$ & 7 & 2.2 & 0.9 & 5.2 \\
\hline -Non-Psychotic Disorders & $300-302,306-316$ & 7 & 2.2 & 0.9 & 5.2 \\
\hline NERVOUS SYSTEM (NS) \& SENSE ORGANS & $320-389$ & 9 & 3.4 & 1.7 & 6.9 \\
\hline -Other Disorders of Central NS & $340-349$ & 1 & 0.4 & 0.1 & 2.8 \\
\hline -Disorders of Peripheral NS & $350-359$ & 3 & 0.8 & 0.2 & 2.6 \\
\hline -Disorders of Eye & $360-379$ & 4 & 1.6 & 0.6 & 4.5 \\
\hline -Diseases of Ear \& Mastoid & $380-389$ & 1 & 0.6 & 0.1 & 4.3 \\
\hline CIRCULATORY SYSTEM & $390-459$ & 17 & 6.5 & 3.5 & 12.0 \\
\hline -Ischemic Heart Dis & $410-414$ & 10 & 3.9 & 1.6 & 9.4 \\
\hline -Other Heart Dis & $420-429$ & 3 & 1.0 & 0.3 & 3.2 \\
\hline -Cerebrovascular Dis & $430-438$ & 1 & 0.4 & 0.1 & 2.8 \\
\hline -Dis of Arteries \& Capillaries & $440-449$ & 1 & 0.2 & 0.0 & 1.4 \\
\hline -Dis of Veins, Lymphatics, Other & $451-459$ & 2 & 1.0 & 0.2 & 4.1 \\
\hline RESPIRATORY SYSTEM & $460-519$ & 7 & 3.4 & 1.5 & 8.1 \\
\hline -Other Dis Upper Respiratory Tract & $470-478$ & 5 & 3.0 & 1.2 & 7.9 \\
\hline -Pneumonia \& Influenza & $480-488$ & 1 & 0.2 & 0.0 & 1.4 \\
\hline -Other Respiratory Dis & $510-519$ & 1 & 0.2 & 0.0 & 1.4 \\
\hline DIGESTIVE SYSTEM & $520-579$ & 18 & 6.4 & 3.7 & 11.2 \\
\hline -Oral Cavity, Saliva Glands, Jaw & $520-529$ & 2 & 0.8 & 0.2 & 3.8 \\
\hline -Appendicitis & $540-543$ & 2 & 1.2 & 0.3 & 4.8 \\
\hline -Hernias & $550-553$ & 9 & 3.2 & 1.4 & 7.3 \\
\hline -Other Intestinal Dis & $560-569$ & 4 & 0.8 & 0.3 & 2.1 \\
\hline -Other Digestive Dis & $570-579$ & 1 & 0.4 & 0.1 & 2.8 \\
\hline GENITOURINARY SYSTEM & $580-629$ & 5 & 2.7 & 0.8 & 9.2 \\
\hline
\end{tabular}

(Continued)

*Only those diagnostic categories with at least one occurrence appear in this table.

**Standardized to age distribution of 2000 U.S. population. 


\section{Kansas City Plant 2010}

Absence Data

Appendix K. Age-Adjusted IIIness and Injury Rates by Diagnostic Category*

Part 1. Men

\begin{tabular}{|c|c|c|c|c|c|}
\hline & & $\begin{array}{l}\text { Number of } \\
\text { Diagnoses }\end{array}$ & $\begin{array}{c}\text { Age-Adjusted } \\
\text { Rate per } 1,000 * *\end{array}$ & $\begin{array}{c}\text { Lower } 95 \% \\
\text { Confidence } \\
\text { Limit per 1,000 }\end{array}$ & $\begin{array}{c}\text { Upper } 95 \% \\
\text { Confidence } \\
\text { Limit per } 1,000\end{array}$ \\
\hline Diagnostic Category & ICD-9-CM Code & \multirow[b]{2}{*}{1} & \multirow[b]{2}{*}{0.2} & \multirow[b]{2}{*}{0.0} & \multirow[b]{2}{*}{1.4} \\
\hline -Nephritis, Nephrosis & $580-589$ & & & & \\
\hline -Other Urinary Dis & $590-599$ & 3 & 1.9 & 0.4 & 9.4 \\
\hline -Male Genital Organ Dis & $600-608$ & 1 & 0.6 & 0.1 & 4.3 \\
\hline SKIN AND SUBCUTANEOUS TISSUE & $680-709$ & 2 & 0.8 & 0.2 & 3.8 \\
\hline -Infections & $680-686$ & 1 & 0.2 & 0.0 & 1.4 \\
\hline -Other & 700-709 & 1 & 0.6 & 0.1 & 4.3 \\
\hline $\begin{array}{l}\text { MUSCULOSKELETAL \& CONNECTIVE } \\
\text { TISSUE }\end{array}$ & $710-739$ & 38 & 13.2 & 9.1 & 19.3 \\
\hline -Arthropathies & $710-719$ & 30 & 10.8 & 7.1 & 16.5 \\
\hline -Dorsopathies & $720-724$ & 4 & 1.2 & 0.4 & 3.7 \\
\hline -Rheumatism, Excluding Back & $725-729$ & 3 & 0.6 & 0.2 & 1.8 \\
\hline -Other Dis \& Acquired Deformities & $730-739$ & 1 & 0.6 & 0.1 & 4.3 \\
\hline $\begin{array}{l}\text { SYMPTOMS, SIGNS, \& ILL-DEFINED } \\
\text { CONDITIONS }\end{array}$ & 780-799 & 9 & 3.4 & 1.7 & 7.0 \\
\hline -Symptoms & $780-789$ & 7 & 2.8 & 1.2 & 6.4 \\
\hline -Non-Specific Abnormal Findings & $790-796$ & 2 & 0.6 & 0.1 & 2.6 \\
\hline INJURY \& POISONING & $800-999$ & 28 & 15.9 & 9.7 & 26.2 \\
\hline -Fracture - Upper Limb & $810-819$ & 1 & 1.5 & 0.2 & 10.9 \\
\hline -Fracture - Lower Limb & $820-829$ & 2 & 3.1 & 0.8 & 12.3 \\
\hline -Dislocation & $830-839$ & 5 & 1.8 & 0.6 & 6.0 \\
\hline -Sprains \& Strains - Other & $840-845,848$ & 13 & 7.7 & 3.9 & 15.4 \\
\hline -Intracranial Injury & $850-854$ & 1 & 0.4 & 0.1 & 2.8 \\
\hline -Open Wound - Head, Neck, Trunk & $870-879$ & 1 & 0.2 & 0.0 & 1.4 \\
\hline -Burns & $940-949$ & 1 & 0.4 & 0.1 & 2.8 \\
\hline -Complications \& Unspecified Injuries & 958-959 & 3 & 0.6 & 0.2 & 1.8 \\
\hline -Poisoning - Medicinal/Biological & $960-979$ & 1 & 0.2 & 0.0 & 1.4 \\
\hline Total & & 154 & 63.7 & 52.1 & 77.8 \\
\hline
\end{tabular}

* Only those diagnostic categories with at least one occurrence appear in this table.

**Standardized to age distribution of 2000 U.S. population. 


\section{Kansas City Plant 2010}

Absence Data

Appendix K. Age-Adjusted Illness and Injury Rates by Diagnostic Category*

Part 2. Women

\begin{tabular}{|c|c|c|c|c|c|}
\hline & & $\begin{array}{l}\text { Number of } \\
\text { Diagnoses }\end{array}$ & $\begin{array}{c}\text { Age-Adjusted } \\
\text { Rate per } 1,000 * *\end{array}$ & $\begin{array}{c}\text { Lower } 95 \% \\
\text { Confidence } \\
\text { Limit per 1,000 }\end{array}$ & $\begin{array}{c}\text { Upper } 95 \% \\
\text { Confidence } \\
\text { Limit per 1,000 }\end{array}$ \\
\hline Diagnostic Category & ICD-9-CM Code & & & & \\
\hline INFECTIOUS \& PARASITIC DISEASES (DIS) & 001-139 & 1 & 4.4 & 0.6 & 31.2 \\
\hline -Other Bacterial Dis & 030-041 & 1 & 4.4 & 0.6 & 31.2 \\
\hline MALIGNANT NEOPLASMS & $\begin{array}{l}140-208, \\
209.0-209.3 \\
230-234\end{array}$ & 1 & 0.6 & 0.1 & 4.3 \\
\hline -Breast & 174-175 & 1 & 0.6 & 0.1 & 4.3 \\
\hline BENIGN \& UNCERTAIN NEOPLASMS & \begin{tabular}{|l|}
$209.4-209.6$ \\
$210-229,235-239$
\end{tabular} & 2 & 1.9 & 0.4 & 8.3 \\
\hline ENDOCRINE/METABOLIC/IMMUNITY & $240-279$ & 4 & 3.8 & 1.3 & 10.8 \\
\hline -Thyroid Gland Disorders & $240-246$ & 2 & 2.6 & 0.6 & 10.4 \\
\hline -Other Endocrine Gland Dis & $249-259$ & 1 & 0.6 & 0.1 & 4.3 \\
\hline -Other Metabolic \& Immunity Disorders & $270-279$ & 1 & 0.6 & 0.1 & 4.3 \\
\hline BLOOD \& BLOOD-FORMING ORGANS & $280-289$ & 1 & 0.6 & 0.1 & 4.3 \\
\hline MENTAL DISORDERS & $290-319$ & 6 & 15.1 & 6.5 & 35.2 \\
\hline -Psychoses & 290-299 & 1 & 3.7 & 0.5 & 26.5 \\
\hline -Non-Psychotic Disorders & $300-302,306-316$ & 5 & 11.3 & 4.5 & 28.5 \\
\hline NERVOUS SYSTEM (NS) \& SENSE ORGANS & $320-389$ & 6 & 8.7 & 2.9 & 26.5 \\
\hline -Inflammatory Dis of Central NS & $320-326$ & 1 & 1.9 & 0.3 & 13.5 \\
\hline -Other Disorders of Central NS & $340-349$ & 1 & 0.6 & 0.1 & 4.3 \\
\hline -Disorders of Peripheral NS & $350-359$ & 2 & 1.2 & 0.3 & 4.9 \\
\hline -Disorders of Eye & $360-379$ & 1 & 0.6 & 0.1 & 4.3 \\
\hline -Diseases of Ear \& Mastoid & $380-389$ & 1 & 4.4 & 0.6 & 31.2 \\
\hline CIRCULATORY SYSTEM & $390-459$ & 4 & 3.7 & 1.2 & 11.7 \\
\hline -Ischemic Heart Dis & $410-414$ & 3 & 1.8 & 0.6 & 5.7 \\
\hline -Dis of Veins, Lymphatics, Other & 451-459 & 1 & 1.9 & 0.3 & 13.5 \\
\hline RESPIRATORY SYSTEM & $460-519$ & 5 & 5.1 & 2.0 & 12.8 \\
\hline -Other Dis Upper Respiratory Tract & $470-478$ & 2 & 1.9 & 0.4 & 8.3 \\
\hline -Pneumonia \& Influenza & $480-488$ & 1 & 0.6 & 0.1 & 4.3 \\
\hline -Chronic Obstructive Dis & $490-496$ & 1 & 1.3 & 0.2 & 9.2 \\
\hline -Other Respiratory Dis & $510-519$ & 1 & 1.3 & 0.2 & 9.2 \\
\hline DIGESTIVE SYSTEM & $520-579$ & 11 & 11.9 & 6.0 & 23.5 \\
\hline -Esophagus, Stomach, Duodenum & $530-538$ & 1 & 0.6 & 0.1 & 4.3 \\
\hline -Appendicitis & $540-543$ & 1 & 0.6 & 0.1 & 4.3 \\
\hline -Enteritis, Colitis & $555-558$ & 2 & 1.2 & 0.3 & 4.9 \\
\hline -Other Intestinal Dis & $560-569$ & 1 & 0.6 & 0.1 & 4.3 \\
\hline -Other Digestive Dis & $570-579$ & 6 & 8.8 & 3.7 & 21.0 \\
\hline
\end{tabular}

(Continued)

* Only those diagnostic categories with at least one occurrence appear in this table.

**Standardized to age distribution of 2000 U.S. population. 


\section{Kansas City Plant 2010}

Absence Data

Appendix K. Age-Adjusted IIIness and Injury Rates by Diagnostic Category*

Part 2. Women

\begin{tabular}{|c|c|c|c|c|c|}
\hline & & $\begin{array}{l}\text { Number of } \\
\text { Diagnoses }\end{array}$ & $\begin{array}{c}\text { Age-Adjusted } \\
\text { Rate per } 1,000 * *\end{array}$ & \begin{tabular}{|c|} 
Lower $95 \%$ \\
Confidence \\
Limit per 1,000
\end{tabular} & $\begin{array}{c}\text { Upper } 95 \% \\
\text { Confidence } \\
\text { Limit per } 1,000\end{array}$ \\
\hline Diagnostic Category & ICD-9-CM Code & & & & \\
\hline GENITOURINARY SYSTEM & $580-629$ & 5 & 10.7 & 3.8 & 30.0 \\
\hline -Other Urinary Dis & $590-599$ & 2 & 2.6 & 0.6 & 10.4 \\
\hline -Other Female Disorders & $617-629$ & 3 & 8.1 & 2.2 & 29.4 \\
\hline SKIN AND SUBCUTANEOUS TISSUE & $680-709$ & 2 & 5.0 & 0.9 & 28.4 \\
\hline -Infections & $680-686$ & 1 & 4.4 & 0.6 & 31.2 \\
\hline -Other & 700-709 & 1 & 0.6 & 0.1 & 4.3 \\
\hline $\begin{array}{l}\text { MUSCULOSKELETAL \& CONNECTIVE } \\
\text { TISSUE }\end{array}$ & $710-739$ & 21 & 17.5 & 10.8 & 28.2 \\
\hline -Arthropathies & $710-719$ & 6 & 3.7 & 1.6 & 8.2 \\
\hline -Dorsopathies & $720-724$ & 6 & 3.7 & 1.6 & 8.2 \\
\hline -Rheumatism, Excluding Back & $725-729$ & 8 & 9.5 & 4.5 & 20.2 \\
\hline -Other Dis \& Acquired Deformities & $730-739$ & 1 & 0.6 & 0.1 & 4.3 \\
\hline $\begin{array}{l}\text { SYMPTOMS, SIGNS, \& ILL-DEFINED } \\
\text { CONDITIONS }\end{array}$ & $780-799$ & 8 & 10.1 & 4.2 & 24.3 \\
\hline -Symptoms & 780-789 & 6 & 8.2 & 2.9 & 22.9 \\
\hline -Non-Specific Abnormal Findings & $790-796$ & 2 & 1.9 & 0.4 & 8.3 \\
\hline INJURY \& POISONING & $800-999$ & 9 & 8.1 & 3.8 & 17.3 \\
\hline -Fracture - Skull & $800-804$ & 1 & 0.6 & 0.1 & 4.3 \\
\hline -Fracture - Upper Limb & 810-819 & 1 & 0.6 & 0.1 & 4.3 \\
\hline -Fracture - Lower Limb & 820-829 & 1 & 0.6 & 0.1 & 4.3 \\
\hline -Sprains \& Strains - Back & 846-847 & 1 & 0.6 & 0.1 & 4.3 \\
\hline -Sprains \& Strains - Other & $840-845,848$ & 2 & 1.2 & 0.3 & 4.9 \\
\hline -Complications \& Unspecified Injuries & 958-959 & 1 & 1.9 & 0.3 & 13.5 \\
\hline -Complications of Surgical/Medical Care & 996-999 & 2 & 2.5 & 0.5 & $\overline{11.9}$ \\
\hline Total & & 86 & 107.2 & 81.7 & 140.7 \\
\hline
\end{tabular}

* Only those diagnostic categories with at least one occurrence appear in this table.

**Standardized to age distribution of 2000 U.S. population. 


\section{Kansas City Plant 2010}

\section{Absence Data}

Appendix K. Age-Adjusted IIIness and Injury Rates by Diagnostic Category*

Part 3. Men and Women

\begin{tabular}{|c|c|c|c|c|c|}
\hline & & $\begin{array}{l}\text { Number of } \\
\text { Diagnoses }\end{array}$ & $\begin{array}{c}\text { Age-Adjusted } \\
\text { Rate per } 1,000 * *\end{array}$ & $\begin{array}{c}\text { Lower } 95 \% \\
\text { Confidence } \\
\text { Limit per 1,000 }\end{array}$ & $\begin{array}{c}\text { Upper } 95 \% \\
\text { Confidence } \\
\text { Limit per 1,000 }\end{array}$ \\
\hline Diagnostic Category & ICD-9-CM Code & & & & \\
\hline INFECTIOUS \& PARASITIC DISEASES (DIS) & 001-139 & 2 & 1.0 & 0.2 & 5.3 \\
\hline -Other Bacterial Dis & 030-041 & 2 & 1.0 & 0.2 & 5.3 \\
\hline MALIGNANT NEOPLASMS & $\begin{array}{l}140-208, \\
209.0-209.3 \\
230-234\end{array}$ & 13 & 3.9 & 2.1 & 7.0 \\
\hline -Lip, Oral Cavity, Pharynx & $140-149$ & 1 & 0.5 & 0.1 & 3.3 \\
\hline -Bone, Connective Tissue, Skin & $170-173,176$ & 2 & 0.9 & 0.2 & 3.7 \\
\hline -Breast & 174-175 & 1 & 0.2 & 0.0 & 1.1 \\
\hline -Genitourinary & $179-189$ & 5 & 1.2 & 0.5 & 3.1 \\
\hline -Other \& Unspecified Sites & 195-199, 209.7 & 3 & 0.9 & 0.3 & 3.1 \\
\hline -Lymphatic \& Hematopoietic & $200-208$ & 1 & 0.2 & 0.0 & 1.1 \\
\hline BENIGN \& UNCERTAIN NEOPLASMS & \begin{tabular}{|l|}
$209.4-209.6$ \\
$210-229$, \\
$235-239$
\end{tabular} & 2 & 0.5 & 0.1 & 2.0 \\
\hline ENDOCRINE/METABOLIC/IMMUNITY & $240-279$ & 5 & 1.4 & 0.5 & 3.6 \\
\hline -Thyroid Gland Disorders & $240-246$ & 3 & 1.1 & 0.3 & 3.4 \\
\hline -Other Endocrine Gland Dis & $249-259$ & 1 & 0.2 & 0.0 & 1.1 \\
\hline -Other Metabolic \& Immunity Disorders & $270-279$ & 1 & 0.2 & 0.0 & 1.1 \\
\hline BLOOD \& BLOOD-FORMING ORGANS & $280-289$ & 1 & 0.2 & 0.0 & 1.1 \\
\hline MENTAL DISORDERS & 290-319 & 13 & 5.7 & 2.9 & 11.0 \\
\hline -Psychoses & 290-299 & 1 & 1.1 & 0.2 & 7.7 \\
\hline -Non-Psychotic Disorders & $300-302,306-316$ & 12 & 4.6 & 2.3 & 9.1 \\
\hline NERVOUS SYSTEM (NS) \& SENSE ORGANS & 320-389 & 15 & 4.5 & 2.5 & 8.2 \\
\hline -Inflammatory Dis of Central NS & $320-326$ & 1 & 0.5 & 0.1 & 3.3 \\
\hline -Other Disorders of Central NS & 340-349 & 2 & 0.5 & 0.1 & 2.0 \\
\hline -Disorders of Peripheral NS & $350-359$ & 5 & 0.9 & 0.4 & 2.3 \\
\hline -Disorders of Eye & $360-379$ & 5 & 1.4 & 0.5 & 3.6 \\
\hline -Diseases of Ear \& Mastoid & $380-389$ & 2 & 1.3 & 0.3 & 5.4 \\
\hline CIRCULATORY SYSTEM & $390-459$ & 21 & 5.9 & 3.4 & 10.0 \\
\hline -Ischemic Heart Dis & $410-414$ & 13 & 3.4 & 1.6 & 7.1 \\
\hline -Other Heart Dis & $420-429$ & 3 & 0.8 & 0.2 & 2.5 \\
\hline -Cerebrovascular Dis & $430-438$ & 1 & 0.3 & 0.0 & 2.2 \\
\hline -Dis of Arteries \& Capillaries & $440-449$ & 1 & 0.2 & 0.0 & 1.1 \\
\hline -Dis of Veins, Lymphatics, Other & 451-459 & 3 & 1.2 & 0.4 & 3.9 \\
\hline RESPIRATORY SYSTEM & $460-519$ & 12 & 3.9 & 2.0 & 7.6 \\
\hline -Other Dis Upper Respiratory Tract & $470-478$ & 7 & 2.8 & 1.2 & 6.5 \\
\hline -Pneumonia \& Influenza & $480-488$ & 2 & 0.3 & 0.1 & 1.2 \\
\hline
\end{tabular}

(Continued)

*Only those diagnostic categories with at least one occurrence appear in this table.

**Standardized to age distribution of 2000 U.S. population. 


\section{Kansas City Plant 2010}

\section{Absence Data}

Appendix K. Age-Adjusted IIIness and Injury Rates by Diagnostic Category*

Part 3. Men and Women

\begin{tabular}{|c|c|c|c|c|c|}
\hline & & $\begin{array}{l}\text { Number of } \\
\text { Diagnoses }\end{array}$ & $\begin{array}{l}\text { Age-Adjusted } \\
\text { Rate per } 1,000 * *\end{array}$ & $\begin{array}{c}\text { Lower } 95 \% \\
\text { Confidence } \\
\text { Limit per 1,000 }\end{array}$ & $\begin{array}{c}\text { Upper } 95 \% \\
\text { Confidence } \\
\text { Limit per 1,000 }\end{array}$ \\
\hline Diagnostic Category & ICD-9-CM Code & \multirow[b]{2}{*}{1} & \multirow[b]{2}{*}{0.3} & \multirow[b]{2}{*}{0.0} & \multirow[b]{2}{*}{2.2} \\
\hline -Chronic Obstructive Dis & $490-496$ & & & & \\
\hline -Other Respiratory Dis & 510-519 & 2 & 0.5 & 0.1 & 2.0 \\
\hline DIGESTIVE SYSTEM & $520-579$ & 29 & 7.8 & 5.1 & 12.1 \\
\hline -Oral Cavity, Saliva Glands, Jaw & $520-529$ & 2 & 0.6 & 0.1 & 2.9 \\
\hline -Esophagus, Stomach, Duodenum & 530-538 & 1 & 0.2 & 0.0 & 1.1 \\
\hline -Appendicitis & $540-543$ & 3 & 1.1 & 0.3 & 3.6 \\
\hline -Hernias & $550-553$ & 9 & 2.5 & 1.1 & 5.8 \\
\hline -Enteritis, Colitis & $555-558$ & 2 & 0.3 & 0.1 & 1.2 \\
\hline -Other Intestinal Dis & $560-569$ & 5 & 0.8 & 0.3 & 1.8 \\
\hline -Other Digestive Dis & $570-579$ & 7 & 2.5 & 1.1 & 5.4 \\
\hline GENITOURINARY SYSTEM & $580-629$ & 10 & 4.9 & 2.2 & 10.9 \\
\hline -Nephritis, Nephrosis & 580-589 & 1 & 0.2 & 0.0 & 1.1 \\
\hline -Other Urinary Dis & $590-599$ & 5 & 2.0 & 0.6 & 6.4 \\
\hline -Male Genital Organ Dis & $600-608$ & 1 & 0.5 & 0.1 & 3.3 \\
\hline -Other Female Disorders & 617-629 & 3 & 2.3 & 0.6 & 8.5 \\
\hline SKIN AND SUBCUTANEOUS TISSUE & $680-709$ & 4 & 1.6 & 0.5 & 5.3 \\
\hline -Infections & $680-686$ & 2 & 1.0 & 0.2 & 5.3 \\
\hline -Other & 700-709 & 2 & 0.6 & 0.1 & 2.9 \\
\hline $\begin{array}{l}\text { MUSCULOSKELETAL \& CONNECTIVE } \\
\text { TISSUE }\end{array}$ & $710-739$ & 59 & 14.5 & 10.7 & 19.6 \\
\hline -Arthropathies & 710-719 & 36 & 9.3 & 6.3 & 13.8 \\
\hline -Dorsopathies & 720-724 & 10 & 1.8 & 0.9 & 3.6 \\
\hline -Rheumatism, Excluding Back & 725-729 & 11 & 2.8 & 1.4 & 5.3 \\
\hline -Other Dis \& Acquired Deformities & 730-739 & 2 & 0.6 & 0.1 & 2.9 \\
\hline $\begin{array}{l}\text { SYMPTOMS, SIGNS, \& ILL-DEFINED } \\
\text { CONDITIONS }\end{array}$ & 780-799 & 17 & 5.2 & 2.9 & 9.4 \\
\hline -Symptoms & 780-789 & 13 & 4.3 & 2.2 & 8.5 \\
\hline -Non-Specific Abnormal Findings & $790-796$ & 4 & 0.9 & 0.3 & 2.6 \\
\hline INJURY \& POISONING & 800-999 & 37 & 13.8 & 8.9 & 21.1 \\
\hline -Fracture - Skull & $800-804$ & 1 & 0.2 & 0.0 & 1.1 \\
\hline -Fracture - Upper Limb & 810-819 & 2 & 1.2 & 0.2 & 7.0 \\
\hline -Fracture - Lower Limb & 820-829 & 3 & 2.3 & 0.6 & 8.5 \\
\hline -Dislocation & $830-839$ & 5 & 1.4 & 0.4 & 4.8 \\
\hline -Sprains \& Strains - Back & 846-847 & 1 & 0.2 & 0.0 & 1.1 \\
\hline -Sprains \& Strains - Other & $840-845,848$ & 15 & 6.0 & 3.2 & 11.6 \\
\hline -Intracranial Injury & $850-854$ & 1 & 0.3 & 0.0 & 2.2 \\
\hline
\end{tabular}

(Continued)

*Only those diagnostic categories with at least one occurrence appear in this table.

**Standardized to age distribution of 2000 U.S. population. 
Kansas City Plant 2010

Absence Data

Appendix K. Age-Adjusted Illness and Injury Rates by Diagnostic Category*

Part 3. Men and Women

\begin{tabular}{|c|c|c|c|c|c|}
\hline & & $\begin{array}{l}\text { Number of } \\
\text { Diagnoses }\end{array}$ & $\begin{array}{c}\text { Age-Adjusted } \\
\text { Rate per } 1,000 * *\end{array}$ & $\begin{array}{c}\text { Lower } 95 \% \\
\text { Confidence } \\
\text { Limit per } 1,000\end{array}$ & $\begin{array}{c}\text { Upper } 95 \% \\
\text { Confidence } \\
\text { Limit per 1,000 }\end{array}$ \\
\hline Diagnostic Category & ICD-9-CM Code & \multirow[b]{2}{*}{1} & \multirow[b]{2}{*}{0.2} & \multirow[b]{2}{*}{0.0} & \multirow[b]{2}{*}{1.1} \\
\hline -Open Wound - Head, Neck, Trunk & $870-879$ & & & & \\
\hline -Burns & $940-949$ & 1 & 0.3 & 0.0 & 2.2 \\
\hline -Complications \& Unspecified Injuries & 958-959 & 4 & 0.9 & 0.3 & 2.8 \\
\hline -Poisoning - Medicinal/Biological & $960-979$ & 1 & 0.2 & 0.0 & 1.1 \\
\hline -Complications of Surgical/Medical Care & 996-999 & 2 & 0.6 & 0.1 & 2.9 \\
\hline Total & & 240 & 74.6 & 63.6 & 87.5 \\
\hline
\end{tabular}

*Only those diagnostic categories with at least one occurrence appear in this table.

**Standardized to age distribution of 2000 U.S. population. 
Kansas City Plant 2010

OSHA Data

Appendix L. Number of Workers with at Least One OSHA Event by Gender, Age, and Job Category*

\begin{tabular}{|c|c|c|c|c|c|c|}
\hline \multirow{3}{*}{ Job Category } & \multicolumn{2}{|c|}{ Women } & \multicolumn{3}{|c|}{ Men } & \multirow[b]{3}{*}{ TOTAL } \\
\hline & \multirow{2}{*}{$\begin{array}{c}\begin{array}{c}\text { Age } \\
\text { Group }\end{array} \\
\mathbf{5 0 +}\end{array}$} & \multirow[b]{2}{*}{ TOTAL } & \multicolumn{2}{|c|}{ Age Group } & \multirow[b]{2}{*}{ TOTAL } & \\
\hline & & & $40-49$ & $50+$ & & \\
\hline Administrative Support & 1 & 1 & 0 & 0 & 0 & 1 \\
\hline Technical Support & 0 & 0 & 0 & 1 & 1 & 1 \\
\hline Service & 1 & 1 & 1 & 0 & 1 & 2 \\
\hline Crafts & 0 & 0 & 0 & 3 & 3 & 3 \\
\hline Line Operators & 1 & 1 & 0 & 0 & 0 & 1 \\
\hline TOTAL & 3 & 3 & 1 & 4 & 5 & 8 \\
\hline
\end{tabular}

*Only those job categories and gender/age combinations with at least one OSHA event appear in this table.

Appendix M. Total Number of Workdays Lost or with Restricted Activity from OSHA Events by Gender and Age

\begin{tabular}{|c|c|c|c|c|c|c|c|c|c|}
\hline \multirow[b]{2}{*}{ Age Group } & \multicolumn{3}{|c|}{ Women } & \multicolumn{3}{|c|}{ Men } & \multicolumn{3}{|c|}{ TOTAL } \\
\hline & $\begin{array}{l}\text { Number of } \\
\text { Events }\end{array}$ & $\begin{array}{c}\text { Days } \\
\text { Restricted }\end{array}$ & Days Lost & $\begin{array}{l}\text { Number of } \\
\text { Events }\end{array}$ & $\begin{array}{c}\text { Days } \\
\text { Restricted }\end{array}$ & Days Lost & $\begin{array}{l}\text { Number of } \\
\text { Events }\end{array}$ & $\begin{array}{c}\text { Days } \\
\text { Restricted }\end{array}$ & Days Lost \\
\hline $40-49$ & 0 & 0 & 0 & 1 & 0 & 0 & 1 & 0 & 0 \\
\hline $50+$ & 4 & 50 & 0 & 4 & 35 & 0 & 8 & 85 & 0 \\
\hline TOTAL & 4 & 50 & 0 & 5 & 35 & 0 & 9 & 85 & 0 \\
\hline
\end{tabular}

Appendix N. Total Number of Workdays Lost or with Restricted Activity from OSHA Events by Gender and Job Category*

\begin{tabular}{|c|c|c|c|c|c|c|c|c|c|}
\hline \multirow[b]{2}{*}{ Job Category } & \multicolumn{3}{|c|}{ Women } & \multicolumn{3}{|c|}{ Men } & \multicolumn{3}{|c|}{ TOTAL } \\
\hline & $\begin{array}{l}\text { Number of } \\
\text { Events }\end{array}$ & $\begin{array}{c}\text { Days } \\
\text { Restricted }\end{array}$ & Days Lost & $\begin{array}{l}\text { Number of } \\
\text { Events }\end{array}$ & $\begin{array}{c}\text { Days } \\
\text { Restricted }\end{array}$ & Days Lost & $\begin{array}{l}\text { Number of } \\
\text { Events }\end{array}$ & $\begin{array}{c}\text { Days } \\
\text { Restricted }\end{array}$ & Days Lost \\
\hline Technical Support & 0 & 0 & 0 & 1 & 0 & 0 & 1 & 0 & 0 \\
\hline Service & 1 & 7 & 0 & 1 & 0 & 0 & 2 & 7 & 0 \\
\hline Line Operators & 2 & 43 & 0 & 0 & 0 & 0 & 2 & 43 & 0 \\
\hline TOTAL & 4 & 50 & 0 & 5 & 35 & 0 & 9 & 85 & 0 \\
\hline
\end{tabular}

*Only those job categories with at least one OSHA event appear in this table. 


\section{Kansas City Plant 2010}

OSHA Data

Appendix O. Number of Diagnoses in Each Diagnostic Category by Gender and Age*

\begin{tabular}{|c|c|c|c|c|c|c|c|}
\hline & & \multicolumn{2}{|c|}{ Women } & \multicolumn{3}{|c|}{ Men } & \multirow[b]{3}{*}{ TOTAL } \\
\hline & & \multirow{2}{*}{\begin{tabular}{|c|} 
Age Group \\
$50+$ \\
\end{tabular}} & \multirow[b]{2}{*}{ TOTAL } & \multicolumn{2}{|c|}{ Age Group } & \multirow[b]{2}{*}{ TOTAL } & \\
\hline & & & & $40-49$ & $50+$ & & \\
\hline Diagnostic Category & ICD-9-CM code & & & & & & \\
\hline RESPIRATORY SYSTEM & $460-519$ & 1 & 1 & 0 & 0 & 0 & 1 \\
\hline -Other Respiratory Dis & $510-519$ & 1 & 1 & 0 & 0 & 0 & 1 \\
\hline GENITOURINARY SYSTEM & $580-629$ & 1 & 1 & 0 & 0 & 0 & 1 \\
\hline -Breast Disorders & $610-612$ & 1 & 1 & 0 & 0 & 0 & 1 \\
\hline $\begin{array}{l}\text { MUSCULOSKELETAL \& CONNECTIVE } \\
\text { TISSUE }\end{array}$ & $710-739$ & 4 & 4 & 0 & 3 & 3 & 7 \\
\hline -Arthropathies & $710-719$ & 4 & 4 & 0 & 1 & 1 & 5 \\
\hline -Dorsopathies & $720-724$ & 0 & 0 & 0 & 1 & 1 & 1 \\
\hline -Rheumatism, Excluding Back & $725-729$ & 0 & 0 & 0 & 1 & 1 & 1 \\
\hline $\begin{array}{l}\text { SYMPTOMS, SIGNS, \& ILL-DEFINED } \\
\text { CONDITIONS }\end{array}$ & $780-799$ & 2 & 2 & 0 & 1 & 1 & 3 \\
\hline -Symptoms & $780-789$ & 2 & 2 & 0 & 1 & 1 & 3 \\
\hline INJURY \& POISONING & $800-999$ & 5 & 5 & 1 & 3 & 4 & 9 \\
\hline -Fracture - Upper Limb & $810-819$ & 1 & 1 & 0 & 0 & 0 & 1 \\
\hline -Fracture - Lower Limb & $820-829$ & 1 & 1 & 0 & 0 & 0 & 1 \\
\hline -Sprains \& Strains - Other & $840-845,848$ & 0 & 0 & 1 & 0 & 1 & 1 \\
\hline -Open Wound - Head, Neck, Trunk & $870-879$ & 0 & 0 & 0 & 1 & 1 & 1 \\
\hline -Open Wound - Upper Limb & $880-887$ & 0 & 0 & 0 & 2 & 2 & 2 \\
\hline -Superficial Injury & 910-919 & 3 & 3 & 0 & 0 & 0 & 3 \\
\hline
\end{tabular}

\begin{tabular}{|c|c|c|c|c|c|c|}
\hline & \multicolumn{2}{|c|}{ Women } & \multicolumn{3}{|c|}{ Men } & \multirow[b]{3}{*}{ TOTAL } \\
\hline & Age Group & \multirow[b]{2}{*}{ TOTAL } & \multicolumn{2}{|c|}{ Age Group } & \multirow[b]{2}{*}{ TOTAL } & \\
\hline & $50+$ & & $40-49$ & $50+$ & & \\
\hline Diagnostic Category & \multirow[b]{2}{*}{13} & \multirow[b]{2}{*}{13} & \multirow[b]{2}{*}{1} & \multirow[b]{2}{*}{7} & \multirow[b]{2}{*}{8} & \multirow[b]{2}{*}{21} \\
\hline Total & & & & & & \\
\hline
\end{tabular}

*Only those diagnostic categories and gender/age combinations with at least one OSHA event appear in this table. 


\section{Kansas City Plant 2010}

OSHA Data

Appendix P. Number of Workdays Lost or with Restricted Activity in Each Diagnostic Category by Gender and Age*

\begin{tabular}{|c|c|c|c|}
\hline & & \multicolumn{2}{|c|}{ Women } \\
\hline & & \multicolumn{2}{|c|}{ Age Group } \\
\hline & & \multicolumn{2}{|c|}{$50+$} \\
\hline & & $\begin{array}{c}\text { Days } \\
\text { Restricted }\end{array}$ & Days Lost \\
\hline Diagnostic Category & ICD-9-CM Codes & \multirow[b]{2}{*}{7} & \multirow[b]{2}{*}{0} \\
\hline -Other Respiratory Dis & $510-519$ & & \\
\hline -Breast Disorders & 610-612 & 7 & 0 \\
\hline -Arthropathies & 710-719 & 21 & 0 \\
\hline -Symptoms & $780-789$ & 21 & 0 \\
\hline -Fracture - Upper Limb & $810-819$ & 29 & 0 \\
\hline -Fracture - Lower Limb & $820-829$ & 0 & 0 \\
\hline -Superficial Injury & 910-919 & 36 & $\overline{0}$ \\
\hline
\end{tabular}

\begin{tabular}{|c|c|c|c|c|c|}
\hline & & \multicolumn{4}{|c|}{ Men } \\
\hline & & \multicolumn{4}{|c|}{ Age Group } \\
\hline & & \multicolumn{2}{|c|}{$40-49$} & \multicolumn{2}{|c|}{$50+$} \\
\hline & & $\begin{array}{c}\text { Days } \\
\text { Restricted }\end{array}$ & Days Lost & $\begin{array}{c}\text { Days } \\
\text { Restricted }\end{array}$ & Days Lost \\
\hline Diagnostic Category & ICD-9-CM Codes & \multirow[b]{2}{*}{0} & \multirow[b]{2}{*}{0} & \multirow[b]{2}{*}{35} & \multirow[b]{2}{*}{0} \\
\hline -Arthropathies & 710-719 & & & & \\
\hline -Dorsopathies & $720-724$ & 0 & 0 & 35 & 0 \\
\hline -Rheumatism, Excluding Back & 725-729 & 0 & 0 & 35 & 0 \\
\hline -Symptoms & 780-789 & 0 & 0 & 35 & 0 \\
\hline -Sprains \& Strains - Other & $840-845,848$ & 0 & 0 & 0 & 0 \\
\hline -Open Wound - Head, Neck, Trunk & 870-879 & 0 & 0 & 0 & 0 \\
\hline -Open Wound - Upper Limb & 880-887 & 0 & 0 & 0 & 0 \\
\hline
\end{tabular}

*OSHA events with >1 ICD-9-CM code in the same diagnostic category were counted only once. Only those diagnostic categories and gender/age combinations with at least one occurrence appear in this table. 
Kansas City Plant 2010

OSHA Data

Appendix Q. Number of Occurrences in Each Accident Category by Gender and Age*

\begin{tabular}{|c|c|c|c|c|c|c|c|}
\hline & & \multicolumn{2}{|c|}{ Women } & \multicolumn{3}{|c|}{ Men } & \multirow[b]{3}{*}{ TOTAL } \\
\hline & & \multirow{2}{*}{\begin{tabular}{|c} 
Age Group \\
$50+$
\end{tabular}} & \multirow[b]{2}{*}{ TOTAL } & \multicolumn{2}{|c|}{ Age Group } & \multirow[b]{2}{*}{ TOTAL } & \\
\hline & & & & $40-49$ & $50+$ & & \\
\hline Type of Accident & E Codes & & & & & & \\
\hline Falls & E880-E888 & 3 & 3 & 0 & 1 & 1 & 4 \\
\hline Other Accidents & E916-E928 & 1 & 1 & 1 & 3 & 4 & 5 \\
\hline
\end{tabular}

*Only those accident types and gender/age combinations with at least one occurrence appear in this table. 
Kansas City Plant 2010

OSHA Data

Appendix R. Number of Workdays Lost or with Restricted Activity in Each Accident Category by Gender and Age*

\begin{tabular}{|c|c|c|c|}
\hline & & \multicolumn{2}{|c|}{ Women } \\
\hline & & \multicolumn{2}{|c|}{ Age Group } \\
\hline & & \multicolumn{2}{|c|}{$50+$} \\
\hline & & $\begin{array}{c}\text { Days } \\
\text { Restricted }\end{array}$ & Days Lost \\
\hline Type of Accident & E Codes & \multirow[b]{2}{*}{36} & \multirow[b]{2}{*}{0} \\
\hline Falls & E880-E888 & & \\
\hline Other Accidents & E916-E928 & 14 & 0 \\
\hline
\end{tabular}

\begin{tabular}{|c|c|c|c|c|c|}
\hline & & \multicolumn{4}{|c|}{ Men } \\
\hline & & \multicolumn{4}{|c|}{ Age Group } \\
\hline & & \multicolumn{2}{|c|}{$40-49$} & \multicolumn{2}{|c|}{$50+$} \\
\hline & & $\begin{array}{c}\text { Days } \\
\text { Restricted }\end{array}$ & Days Lost & $\begin{array}{c}\text { Days } \\
\text { Restricted }\end{array}$ & Days Lost \\
\hline Type of Accident & E Codes & \multirow[b]{2}{*}{0} & \multirow[b]{2}{*}{0} & \multirow[b]{2}{*}{35} & \multirow[b]{2}{*}{0} \\
\hline Falls & E880-E888 & & & & \\
\hline Other Accidents & E916-E928 & 0 & 0 & 0 & 0 \\
\hline
\end{tabular}

*OSHA events with $>1 \mathrm{E}$ code in the same accident type were counted only once. Only those accident types and gender/age combinations with at least one occurrence appear in this table. 


\section{Kansas City Plant 2010}

OSHA Data

Appendix S. Number of Diagnoses in Each Diagnostic Category by Gender and Job Category*

\begin{tabular}{|c|c|c|c|c|c|}
\hline & & \multicolumn{4}{|c|}{ Women } \\
\hline & & \multicolumn{3}{|c|}{ Job Category } & \multirow[b]{2}{*}{ TOTAL } \\
\hline & & $\begin{array}{l}\text { Administrative } \\
\text { Support }\end{array}$ & Service & Line Operators & \\
\hline Diagnostic Category & ICD-9-CM Code & & & & \\
\hline $\begin{array}{l}\text { RESPIRATORY } \\
\text { SYSTEM }\end{array}$ & $460-519$ & 0 & 1 & 0 & 1 \\
\hline -Other Respiratory Dis & $510-519$ & 0 & 1 & 0 & 1 \\
\hline $\begin{array}{l}\text { GENITOURINARY } \\
\text { SYSTEM }\end{array}$ & $580-629$ & 0 & 1 & 0 & 1 \\
\hline -Breast Disorders & $610-612$ & 0 & 1 & 0 & 1 \\
\hline $\begin{array}{l}\text { MUSCULOSKELETAL } \\
\text { \& CONNECTIVE } \\
\text { TISSUE }\end{array}$ & $710-739$ & 1 & 2 & 1 & 4 \\
\hline -Arthropathies & $710-719$ & 1 & 2 & 1 & 4 \\
\hline $\begin{array}{l}\text { SYMPTOMS, } \\
\text { SIGNS, \& ILL-DEFINED } \\
\text { CONDITIONS } \\
\end{array}$ & $780-799$ & 0 & 1 & 1 & 2 \\
\hline -Symptoms & \begin{tabular}{|l|}
$780-789$ \\
\end{tabular} & 0 & 1 & 1 & 2 \\
\hline INJURY \& POISONING & $800-999$ & 1 & 1 & 3 & 5 \\
\hline -Fracture - Upper Limb & $810-819$ & 0 & 0 & 1 & 1 \\
\hline -Fracture - Lower Limb & $820-829$ & 1 & 0 & 0 & 1 \\
\hline -Superficial Injury & 910-919 & 0 & 1 & 2 & 3 \\
\hline
\end{tabular}

\begin{tabular}{|c|c|c|c|c|}
\hline & \multicolumn{4}{|c|}{ Women } \\
\hline & \multicolumn{3}{|c|}{ Job Category } & \multirow[b]{2}{*}{ TOTAL } \\
\hline & $\begin{array}{l}\text { Administrative } \\
\text { Support }\end{array}$ & Service & Line Operators & \\
\hline Diagnostic Category & \multirow[b]{2}{*}{2} & \multirow[b]{2}{*}{6} & \multirow[b]{2}{*}{5} & \multirow[b]{2}{*}{13} \\
\hline Total & & & & \\
\hline
\end{tabular}

\begin{tabular}{|c|c|c|c|c|c|}
\hline & & \multicolumn{4}{|c|}{ Men } \\
\hline & & \multicolumn{3}{|c|}{ Job Category } & \multirow[b]{2}{*}{ TOTAL } \\
\hline & & Technical Support & Service & Crafts & \\
\hline Diagnostic Category & ICD-9-CM Code & & & & \\
\hline $\begin{array}{l}\text { MUSCULOSKELETAL } \\
\text { \& CONNECTIVE } \\
\text { TISSUE }\end{array}$ & 710-739 & 0 & 0 & 3 & 3 \\
\hline -Arthropathies & $710-719$ & 0 & 0 & 1 & 1 \\
\hline -Dorsopathies & $720-724$ & 0 & 0 & 1 & 1 \\
\hline $\begin{array}{l}\text {-Rheumatism, Excluding } \\
\text { Back }\end{array}$ & 725-729 & 0 & 0 & 1 & 1 \\
\hline $\begin{array}{l}\text { SYMPTOMS, } \\
\text { SIGNS, \& ILL-DEFINED } \\
\text { CONDITIONS }\end{array}$ & $780-799$ & 0 & 0 & 1 & 1 \\
\hline -Symptoms & $780-789$ & 0 & 0 & 1 & 1 \\
\hline
\end{tabular}

(Continued)

*Only those diagnostic categories and gender/job category combinations with at least one occurrence appear in this table. 
Kansas City Plant 2010

OSHA Data

Appendix S. Number of Diagnoses in Each Diagnostic Category by Gender and Job Category*

\begin{tabular}{|c|c|c|c|c|c|}
\hline & & \multicolumn{4}{|c|}{ Men } \\
\hline & & \multicolumn{3}{|c|}{ Job Category } & \multirow[b]{2}{*}{ TOTAL } \\
\hline & & Technical Support & Service & Crafts & \\
\hline Diagnostic Category & ICD-9-CM Code & \multirow[b]{2}{*}{1} & \multirow[b]{2}{*}{1} & \multirow[b]{2}{*}{2} & \multirow[b]{2}{*}{4} \\
\hline INJURY \& POISONING & $800-999$ & & & & \\
\hline $\begin{array}{l}\text {-Sprains \& Strains - } \\
\text { Other }\end{array}$ & $840-845,848$ & 0 & 1 & 0 & 1 \\
\hline $\begin{array}{l}\text {-Open Wound - Head, } \\
\text { Neck, Trunk }\end{array}$ & 870-879 & 0 & 0 & 1 & 1 \\
\hline $\begin{array}{l}\text {-Open Wound - Upper } \\
\text { Limb }\end{array}$ & $880-887$ & 1 & 0 & 1 & 2 \\
\hline
\end{tabular}

\begin{tabular}{|c|c|c|c|c|}
\hline & \multicolumn{4}{|c|}{ Men } \\
\hline & \multicolumn{3}{|c|}{ Job Category } & \multirow[b]{2}{*}{ TOTAL } \\
\hline & Technical Support & Service & Crafts & \\
\hline Diagnostic Category & \multirow[b]{2}{*}{1} & \multirow[b]{2}{*}{1} & \multirow[b]{2}{*}{6} & \multirow[b]{2}{*}{8} \\
\hline Total & & & & \\
\hline
\end{tabular}

*Only those diagnostic categories and gender/job category combinations with at least one occurrence appear in this table. 


\section{Kansas City Plant 2010}

OSHA Data

Appendix T. Number of Workdays Lost or with Restricted Activity in Each Diagnostic Category by Gender and Job Category*

\begin{tabular}{|c|c|c|c|c|c|c|c|}
\hline & & \multicolumn{6}{|c|}{ Women } \\
\hline & & \multicolumn{6}{|c|}{ Job Category } \\
\hline & & \multicolumn{2}{|c|}{$\begin{array}{c}\text { Administrative } \\
\text { Support }\end{array}$} & \multicolumn{2}{|c|}{ Service } & \multicolumn{2}{|c|}{ Line Operators } \\
\hline & & $\begin{array}{c}\text { Days } \\
\text { Restricted }\end{array}$ & Days Lost & $\begin{array}{c}\text { Days } \\
\text { Restricted }\end{array}$ & Days Lost & \begin{tabular}{|c|} 
Days \\
Restricted
\end{tabular} & Days Lost \\
\hline Diagnostic Category & ICD-9-CM Codes & \multirow[b]{2}{*}{0} & \multirow[b]{2}{*}{0} & \multirow[b]{2}{*}{7} & \multirow[b]{2}{*}{0} & \multirow[b]{2}{*}{0} & \multirow[b]{2}{*}{0} \\
\hline -Other Respiratory Dis & $510-519$ & & & & & & \\
\hline -Breast Disorders & $610-612$ & 0 & 0 & 7 & 0 & 0 & 0 \\
\hline -Arthropathies & $710-719$ & 0 & 0 & 7 & 0 & 14 & $\overline{0}$ \\
\hline -Symptoms & $780-789$ & 0 & 0 & 7 & 0 & 14 & 0 \\
\hline -Fracture - Upper Limb & 810-819 & 0 & 0 & 0 & 0 & 29 & 0 \\
\hline -Fracture - Lower Limb & $820-829$ & 0 & 0 & 0 & 0 & 0 & 0 \\
\hline -Superficial Injury & $910-919$ & 0 & 0 & 7 & 0 & 29 & 0 \\
\hline
\end{tabular}

\begin{tabular}{|c|c|c|c|c|c|c|c|}
\hline & \multicolumn{6}{|c|}{ Men } \\
\hline & & \multicolumn{6}{|c|}{ Job Category } \\
\hline & & \multicolumn{2}{|c|}{ Technical Support } & \multicolumn{2}{|c|}{ Service } & \multicolumn{2}{|c|}{ Crafts } \\
\hline & & $\begin{array}{c}\text { Days } \\
\text { Restricted }\end{array}$ & Days Lost & \begin{tabular}{|c|} 
Days \\
Restricted
\end{tabular} & Days Lost & \begin{tabular}{c|} 
Days \\
Restricted
\end{tabular} & Days Lost \\
\hline Diagnostic Category & ICD-9-CM Codes & & & & & & \\
\hline -Arthropathies & $710-719$ & 0 & 0 & 0 & 0 & 35 & 0 \\
\hline -Dorsopathies & $720-724$ & 0 & 0 & 0 & 0 & 35 & 0 \\
\hline -Rheumatism, Excluding Back & $725-729$ & 0 & 0 & 0 & 0 & 35 & 0 \\
\hline -Symptoms & $780-789$ & 0 & 0 & 0 & 0 & 35 & 0 \\
\hline -Sprains \& Strains - Other & $840-845,848$ & 0 & 0 & 0 & 0 & 0 & 0 \\
\hline -Open Wound - Head, Neck, Trunk & $870-879$ & 0 & 0 & 0 & 0 & 0 & 0 \\
\hline -Open Wound - Upper Limb & $880-887$ & 0 & 0 & 0 & 0 & 0 & 0 \\
\hline
\end{tabular}

*OSHA events with >1 ICD-9-CM code in the same diagnostic category were counted only once. Only those diagnostic categories and gender/job category combinations with at least one occurrence appear in this table. 
Kansas City Plant 2010

OSHA Data

Appendix U. Number of Occurrences in Each Accident Category by Gender and Job Category*

\begin{tabular}{|c|c|c|c|c|c|}
\hline & & \multicolumn{4}{|c|}{ Women } \\
\hline & & \multicolumn{3}{|c|}{ Job Category } & \multirow[b]{2}{*}{ TOTAL } \\
\hline & & $\begin{array}{c}\text { Administrative } \\
\text { Support }\end{array}$ & Service & $\begin{array}{c}\text { Line } \\
\text { Operators }\end{array}$ & \\
\hline Type of Accident & E CODES & \multirow[b]{2}{*}{1} & \multirow[b]{2}{*}{1} & \multirow[b]{2}{*}{1} & \multirow[b]{2}{*}{3} \\
\hline Falls & \begin{tabular}{|l|} 
E880-E888 \\
\end{tabular} & & & & \\
\hline Other Accidents & E916-E928 & 0 & 0 & 1 & 1 \\
\hline
\end{tabular}

\begin{tabular}{|c|c|c|c|c|c|}
\hline & & \multicolumn{4}{|c|}{ Men } \\
\hline & & \multicolumn{3}{|c|}{ Job Category } & \multirow[b]{2}{*}{ TOTAL } \\
\hline & & $\begin{array}{l}\text { Technical } \\
\text { Support }\end{array}$ & Service & Crafts & \\
\hline Type of Accident & E CODES & \multirow[b]{2}{*}{0} & \multirow[b]{2}{*}{0} & \multirow[b]{2}{*}{1} & \multirow[b]{2}{*}{1} \\
\hline Falls & E880-E888 & & & & \\
\hline Other Accidents & E916-E928 & 1 & 1 & 2 & 4 \\
\hline
\end{tabular}

*Only those accident types and gender/job category combinations with at least one occurrence appear in this table. 
Kansas City Plant 2010

OSHA Data

Appendix V. Number of Workdays Lost or with Restricted Activity in Each Accident Category by Gender and Job Category*

\begin{tabular}{|c|c|c|c|c|c|c|c|}
\hline & & \multicolumn{6}{|c|}{ Women } \\
\hline & & \multicolumn{6}{|c|}{ Job Category } \\
\hline & & \multicolumn{2}{|c|}{$\begin{array}{l}\text { Administrative } \\
\text { Support }\end{array}$} & \multicolumn{2}{|c|}{ Service } & \multicolumn{2}{|c|}{ Line Operators } \\
\hline & & $\begin{array}{c}\text { Days } \\
\text { Restricted }\end{array}$ & Days Lost & $\begin{array}{c}\text { Days } \\
\text { Restricted }\end{array}$ & Days Lost & $\begin{array}{c}\text { Days } \\
\text { Restricted }\end{array}$ & Days Lost \\
\hline Type of Accident & E Codes & \multirow[b]{2}{*}{0} & \multirow[b]{2}{*}{0} & \multirow[b]{2}{*}{7} & \multirow[b]{2}{*}{0} & \multirow[b]{2}{*}{29} & \multirow[b]{2}{*}{0} \\
\hline Falls & E880-E888 & & & & & & \\
\hline Other Accidents & E916-E928 & 0 & 0 & 0 & 0 & 14 & 0 \\
\hline
\end{tabular}

\begin{tabular}{|c|c|c|c|c|c|c|c|}
\hline & \multicolumn{6}{|c|}{ Men } \\
\hline & & \multicolumn{6}{|c|}{ Job Category } \\
\hline & & \multicolumn{2}{|c|}{ Technical Support } & \multicolumn{2}{|c|}{ Service } & \multicolumn{2}{|c|}{ Crafts } \\
\hline & & \begin{tabular}{c|} 
Days \\
Restricted
\end{tabular} & Days Lost & \begin{tabular}{|c|} 
Days \\
Restricted
\end{tabular} & Days Lost & \begin{tabular}{|c|} 
Days \\
Restricted
\end{tabular} & Days Lost \\
\hline Type of Accident & E Codes & \multirow[b]{2}{*}{0} & \multirow[b]{2}{*}{0} & \multirow[b]{2}{*}{0} & \multirow[b]{2}{*}{0} & \multirow[b]{2}{*}{35} & \multirow[b]{2}{*}{0} \\
\hline Falls & E880-E888 & & & & & & \\
\hline Other Accidents & E916-E928 & 0 & 0 & 0 & 0 & 0 & 0 \\
\hline
\end{tabular}

*OSHA events with >1 E code in the same accident type were counted only once. Only those accident types and gender/job category combinations with at least one occurrence appear in this table. 
Kansas City Plant 2010

OSHA Data

Appendix W. Age-Adjusted OSHA Illness and Injury Rates by Diagnostic Category*

Part 1. Men

\begin{tabular}{|c|c|c|c|c|c|}
\hline & & $\begin{array}{l}\text { Number of } \\
\text { Diagnoses }\end{array}$ & $\begin{array}{c}\text { Age-Adjusted } \\
\text { Rate per } 1,000 * *\end{array}$ & $\begin{array}{c}\text { Lower } 95 \% \\
\text { Confidence } \\
\text { Limit per 1,000 }\end{array}$ & $\begin{array}{c}\text { Upper } 95 \% \\
\text { Confidence } \\
\text { Limit per } 1,000\end{array}$ \\
\hline Diagnostic Category & ICD-9-CM Code & & & & \\
\hline $\begin{array}{l}\text { MUSCULOSKELETAL \& CONNECTIVE } \\
\text { TISSUE }\end{array}$ & 710-739 & 3 & 0.6 & 0.2 & 1.8 \\
\hline -Arthropathies & $710-719$ & 1 & 0.2 & 0.0 & 1.4 \\
\hline -Dorsopathies & $720-724$ & 1 & 0.2 & 0.0 & 1.4 \\
\hline -Rheumatism, Excluding Back & $725-729$ & 1 & 0.2 & 0.0 & 1.4 \\
\hline $\begin{array}{l}\text { SYMPTOMS, SIGNS, \& ILL-DEFINED } \\
\text { CONDITIONS }\end{array}$ & $780-799$ & 1 & 0.2 & 0.0 & 1.4 \\
\hline -Symptoms & 780-789 & 1 & 0.2 & 0.0 & 1.4 \\
\hline INJURY \& POISONING & $800-999$ & 4 & 1.2 & 0.4 & 3.7 \\
\hline -Sprains \& Strains - Other & $840-845,848$ & 1 & 0.6 & 0.1 & 4.3 \\
\hline -Open Wound - Head, Neck, Trunk & $870-879$ & 1 & 0.2 & 0.0 & 1.4 \\
\hline -Open Wound - Upper Limb & $880-887$ & 2 & 0.4 & 0.1 & 1.6 \\
\hline Total & & 8 & 2.0 & 0.9 & 4.4 \\
\hline
\end{tabular}

* Only those diagnostic categories with at least one occurrence appear in this table.

**Standardized to age distribution of 2000 U.S. population. 
Kansas City Plant 2010

OSHA Data

Appendix W. Age-Adjusted OSHA Illness and Injury Rates by Diagnostic Category*

Part 2. Women

\begin{tabular}{|c|c|c|c|c|c|}
\hline & & $\begin{array}{l}\text { Number of } \\
\text { Diagnoses }\end{array}$ & $\begin{array}{c}\text { Age-Adjusted } \\
\text { Rate per } 1,000 * *\end{array}$ & $\begin{array}{c}\text { Lower } 95 \% \\
\text { Confidence } \\
\text { Limit per 1,000 }\end{array}$ & $\begin{array}{c}\text { Upper } 95 \% \\
\text { Confidence } \\
\text { Limit per } 1,000\end{array}$ \\
\hline Diagnostic Category & ICD-9-CM Code & \multirow[b]{2}{*}{1} & \multirow[b]{2}{*}{0.6} & \multirow[b]{2}{*}{0.1} & \multirow[b]{2}{*}{4.3} \\
\hline RESPIRATORY SYSTEM & $460-519$ & & & & \\
\hline -Other Respiratory Dis & $510-519$ & 1 & 0.6 & 0.1 & 4.3 \\
\hline GENITOURINARY SYSTEM & $580-629$ & 1 & 0.6 & 0.1 & 4.3 \\
\hline -Breast Disorders & $610-612$ & 1 & 0.6 & 0.1 & 4.3 \\
\hline $\begin{array}{l}\text { MUSCULOSKELETAL \& CONNECTIVE } \\
\text { TISSUE }\end{array}$ & $710-739$ & 4 & 2.4 & 0.9 & 6.5 \\
\hline -Arthropathies & $710-719$ & 4 & 2.4 & 0.9 & 6.5 \\
\hline $\begin{array}{l}\text { SYMPTOMS, SIGNS, \& ILL-DEFINED } \\
\text { CONDITIONS }\end{array}$ & 780-799 & 2 & 1.2 & 0.3 & 4.9 \\
\hline -Symptoms & $780-789$ & 2 & 1.2 & 0.3 & 4.9 \\
\hline INJURY \& POISONING & $800-999$ & 5 & 3.1 & 1.3 & 7.4 \\
\hline -Fracture - Upper Limb & $810-819$ & 1 & 0.6 & 0.1 & 4.3 \\
\hline -Fracture - Lower Limb & \begin{tabular}{|l|}
$820-829$ \\
\end{tabular} & 1 & 0.6 & 0.1 & 4.3 \\
\hline -Superficial Injury & 910-919 & 3 & 1.8 & 0.6 & 5.7 \\
\hline Total & & 13 & 8.0 & 4.6 & 13.7 \\
\hline
\end{tabular}

* Only those diagnostic categories with at least one occurrence appear in this table.

**Standardized to age distribution of 2000 U.S. population. 
Kansas City Plant 2010

OSHA Data

Appendix W. Age-Adjusted OSHA Illness and Injury Rates by Diagnostic Category*

Part 3. Men and Women

\begin{tabular}{|c|c|c|c|c|c|}
\hline & & $\begin{array}{l}\text { Number of } \\
\text { Diagnoses }\end{array}$ & $\begin{array}{c}\text { Age-Adjusted } \\
\text { Rate per } 1,000 * *\end{array}$ & $\begin{array}{c}\text { Lower } 95 \% \\
\text { Confidence } \\
\text { Limit per } 1,000\end{array}$ & $\begin{array}{c}\text { Upper } 95 \% \\
\text { Confidence } \\
\text { Limit per 1,000 }\end{array}$ \\
\hline Diagnostic Category & ICD-9-CM Code & \multirow[b]{2}{*}{1} & \multirow[b]{2}{*}{0.2} & \multirow[b]{2}{*}{0.0} & \multirow[b]{2}{*}{1.1} \\
\hline RESPIRATORY SYSTEM & $460-519$ & & & & \\
\hline -Other Respiratory Dis & $510-519$ & 1 & 0.2 & 0.0 & 1.1 \\
\hline GENITOURINARY SYSTEM & $580-629$ & 1 & 0.2 & 0.0 & 1.1 \\
\hline -Breast Disorders & $610-612$ & 1 & 0.2 & 0.0 & 1.1 \\
\hline $\begin{array}{l}\text { MUSCULOSKELETAL \& CONNECTIVE } \\
\text { TISSUE }\end{array}$ & $710-739$ & 7 & 1.1 & 0.5 & 2.2 \\
\hline -Arthropathies & $710-719$ & 5 & 0.8 & 0.3 & 1.8 \\
\hline -Dorsopathies & $720-724$ & 1 & 0.2 & 0.0 & 1.1 \\
\hline -Rheumatism, Excluding Back & 725-729 & 1 & 0.2 & 0.0 & 1.1 \\
\hline $\begin{array}{l}\text { SYMPTOMS, SIGNS, \& ILL-DEFINED } \\
\text { CONDITIONS }\end{array}$ & 780-799 & 3 & 0.5 & 0.1 & 1.4 \\
\hline -Symptoms & $780-789$ & 3 & 0.5 & 0.1 & 1.4 \\
\hline INJURY \& POISONING & $800-999$ & 9 & 1.7 & 0.8 & 3.5 \\
\hline -Fracture - Upper Limb & 810-819 & 1 & 0.2 & 0.0 & 1.1 \\
\hline -Fracture - Lower Limb & 820-829 & 1 & 0.2 & 0.0 & 1.1 \\
\hline -Sprains \& Strains - Other & $840-845,848$ & 1 & 0.5 & 0.1 & 3.3 \\
\hline -Open Wound - Head, Neck, Trunk & 870-879 & 1 & 0.2 & 0.0 & 1.1 \\
\hline -Open Wound - Upper Limb & 880-887 & 2 & 0.3 & 0.1 & 1.2 \\
\hline -Superficial Injury & $910-919$ & 3 & 0.5 & 0.1 & 1.4 \\
\hline Total & & 21 & 3.5 & 2.2 & 5.5 \\
\hline
\end{tabular}

* Only those diagnostic categories with at least one occurrence appear in this table.

**Standardized to age distribution of 2000 U.S. population. 